\title{
A single theory for some quasi-static, supersonic, atomic, and tectonic scale applications of dislocations
}

\author{
Xiaohan Zhang, Amit Acharya, Noel J. Walkington, Jacobo Bielak
}

May 24, 2015

\begin{abstract}
We describe a model based in continuum mechanics that reduces the study of a significant class of problems of discrete dislocation dynamics to questions of the modern theory of continuum plasticity. As applications, we explore the questions of the existence of a Peierls stress in a continuum theory, dislocation annihilation, dislocation dissociation, finite-speed-of-propagation effects of elastic waves vis-a-vis dynamic dislocation fields, supersonic dislocation motion, and short-slip duration in rupture dynamics.
\end{abstract}

\section{Introduction}

This paper explores some qualitative aspects of Field Dislocation Mechanics (FDM), a nonlinear, partial differential equation (pde)-based model of the mechanics of dislocations. The physical phenomena explored correspond to behaviour of individual or a collection of few dislocations. In particular, we analyse phenomena complementary to what can be dealt with by the Discrete Dislocation Dynamics methodology in a fundamental manner. Specifically, we explore

- Peierls' stress effects in a translationally-invariant continuum theory like FDM.

- Dislocation annihilation and dissociation as consequences of fundamental kinematics and energetics and not targeted constitutive rules for the phenomena.

- Dislocation dynamics in the presence of significant effects of material inertia, including finite-speed-of-propagation effects of elastic waves and dislocation motion past sonic speeds.

- Dislocation dynamics with nonlinear elasticity.

- Short-slip duration in rupture dynamics.

The question of the possibility of a Peierls-like threshold for onset of dislocation motion in a translationally-invariant, time-dependent continuum theory was discussed in [Ach10]. The classical, static, argument going back to Peierls [Pei40] relies crucially on the fact that such a threshold is directly related to changes in the total potential energy of the body 
induced by changes in position of the dislocation (naturally, then, viewed as a rigid object or profile). Since in a homogeneous infinite continuum the total potential energy remains invariant due to changes in position of the rigid dislocation profile, the conclusion is that there cannot be a Peierls stress in a translationally-invariant continuum theory; breaking translational invariance, possibly by modelling the effects of an atomic lattice (as was done by Peierls [Pei40] and Nabarro [Nab47]) or by introducing a heterogeneous medium, can introduce a Peierls stress. However, questions of stability of equilibria under perturbations of loading in a time-dependent model of dislocation mechanics with a significantly different notion of a driving force (that includes self stress effects) can be quite different, in particular whether an unloaded equilibrium dislocation profile can serve as a traveling wave profile under a continuous spectrum of finite loads tending to zero - and, if not, is there an interval of loads about zero for which different equilibrium profiles can be attained parametrized by the load. Such questions have to do intimately with changes in 'shape' of the dislocation profile. In this paper we computationally explore this question - as a point of principle, most of all - for three natural models that the structure of FDM makes available. These correspond to a non-local Ginzburg Landau model, a non-local level set model and what may be termed a generalized, non-local Burgers model. In Sections 4 and 7.1 we describe these models and results in detail. Despite the great utility of analysis of traveling waves, our results point definitely in the direction of avoiding an over-reliance on characteristics of traveling wave solutions in making general statements about the non-existence of certain types of predictions related to the representation of physical phenomena characterized by fronts. After all, there is no reason why a traveling front necessarily has to be perfectly rigid during motion, making an infinite-dimensional object (a profile allowed to change shape, while still remaining localized) into one of dimension 1. A physical example related to this paper is the onset of motion of screw dislocations in some BCC materials. There, it is understood that the dislocation core is spread out on multiple planes and the core has to be compacted further into a preferred slip plane before gross motion can ensue; once motion stops, the multiple-plane equilibrium configuration is regained. In a qualitative sense, we demonstrate such features, including differences between dynamic and equilibrium shapes in Section 7.

With respect to dislocation annihilation, since the fundamental statement of evolution in FDM is a conservation law for Burgers vector content of the dislocation density field, the density field evolves by tensorial addition rules resulting in natural accumulation or annihilation of non-singular localizations of net positive and negative Burgers vector when physically expected. We demonstrate such results in Section 7.4.

The discussion of the possible dissociation of a dislocation of a certain Burgers vector strength into two whose strengths vectorially sum up to that of the original one is a textbook example of the phenomenology of dislocations related to the energy-decreasing feature of dislocation mechanics. Due to the treatment of a dislocation core as either a formless or a rigid singularity in classical versions of any sort of dislocation dynamics, dissociation cannot be a prediction. The field setting is ideally suited for such explorations as we demonstrate in Sec. 7.1.7.

As for dislocation dynamics with material inertia, it is physically natural that a moving dislocation induces elastic stress-waves that cannot transmit the stress signal instantaneously to all parts of the body. This fact is naturally encoded in FDM and our simulations, without 
extra effort or computational expense beyond solving standard elastodynamics equations. As discussed in [GLBD $\left.{ }^{+} 13\right]$, when time intervals of observation are small (as in very high rate deformations) this time delay in stress signal transmission due to stress-wave propagation can be of importance, and merely correcting for individual dislocation motion laws in DD simulations by added-mass effects, while utilizing the static stress fields of dislocations, is not sufficient; instead, dislocation stress fields utilizing the full dynamic Green's function have to be utilized and this becomes a significantly onerous task, especially with increase in number of segments. We demonstrate the efficacy of FDM in dealing with such problems in Section 8.3. In addition, we show that there is no conceptual or practical problem within FDM in dealing with dislocation motion past linear-elastic sonic speeds (in appropriate circumstances) as observed in the molecular dynamics (MD) experiments of [GG99], or in dealing with nonlinear elasticity, with beneficial effect related to matching trends of dislocation velocity vs. applied loading to MD results.

Finally, we make a successful first attempt at modeling the observed phenomenon of short-slip duration in earthquake rupture as well as the more conventional crack-like slip response obtained from slip-weakening cohesive zone models of rupture dynamics. These features are obtained without sophisticated constitutive modifications of velocity-weakening or rate-and-state friction type, but simply by invoking a requirement of damage of elastic modulus at a point on propagation of the rupture front past it.

In this work we utilize an ansatz to produce an exact, reduced, plane model of FDM. Our model is built on the previous work of [Ach10] where a 1-d FDM model was derived and further explored numerically in [DAZM13]. The 1-D model, taking the form of a nonlinear Hamilton-Jacobi equation, governs the evolution of plastic shear strain in a 1-d bar. Mathematical analysis of traveling waves in the model for the scalar case was performed in [AMZ10]; global existence and uniqueness for the 1-d space $\times$ time system was analysed in [AT11]. Our work generalizes the 1-D model to plane strain where edge dislocations exist and glide horizontally along a prescribed plastic layer. The plastic evolution is governed by a similar 1-d model as derived in [Ach10], but now nonlocal if viewed solely as an equation in terms of the plastic strain, with the 3 - $\mathrm{d}$ dissipation maintained non-negative without approximation. This results in a useful model that is amenable to reasonably efficient and accurate numerical simulation.

The rest of the paper is organized as follows: In Section 2 we settle on notational conventions. In Section 3 we briefly recall the full 3-D FDM theory in the geometrically linear framework. We describe the derivation of the 2-d model in Section 4. The numerical schemes utilized in the paper are described in Section 5. Equilibrium aspects of the system are dis-

cussed in Section 6. Features of the model related to dislocation motion under quasi-static deformations are presented in Section 7 and results on dynamics with inertia are presented in Section 8. We end with some concluding remarks in Section 9.

\section{Notation}

Vectors are represented by boldface letters. A superposed dot represents a material time derivative. A subscript $x$ or $t$ represents partial differentiation with respect to $x$ or $t$, respectively. The summation convention is implied. A second (or higher) order tensor $\boldsymbol{A}$ acting 
on a vector $\boldsymbol{v}$ is denoted by $\boldsymbol{A} \boldsymbol{v}$ and the inner product of two second-order tensors $\boldsymbol{A}$ and $\boldsymbol{B}$ is represented by $\boldsymbol{A}: \boldsymbol{B}$. The indicial form with respect to rectangular Cartesian bases are $\boldsymbol{A} \boldsymbol{v}=A_{i j} v_{j}$ and $\boldsymbol{A} \boldsymbol{B}=A_{i j} B_{i j}$ respectively. Let $\boldsymbol{c}$ be a spatially constant vector field; the cross product, divergence, and curl are defined as

$$
\begin{aligned}
& (\boldsymbol{A} \times \boldsymbol{v})^{T} \boldsymbol{c}=\left(\boldsymbol{A}^{T} \boldsymbol{c}\right) \times \boldsymbol{v} \quad \forall \boldsymbol{c} \\
& (\operatorname{div} \boldsymbol{A}) \boldsymbol{c}=\operatorname{div}\left(\boldsymbol{A}^{T} \boldsymbol{c}\right) \quad \forall \boldsymbol{c} \\
& (\operatorname{curl} \boldsymbol{A})^{T} \boldsymbol{c}=\operatorname{curl}\left(\boldsymbol{A}^{T} \boldsymbol{c}\right) \quad \forall \boldsymbol{c}
\end{aligned}
$$

with component representation with respect to rectangular Cartesian coordinate systems given by

$$
\begin{aligned}
& (\boldsymbol{A} \times \boldsymbol{v})_{i m}=e_{m j k} A_{i j} v_{k} \\
& (\operatorname{div} \boldsymbol{A})_{i}=A_{i j, j} \\
& (\operatorname{curl} \boldsymbol{A})_{i m}=e_{m j k} A_{i k, j},
\end{aligned}
$$

where $e_{m j k}$ are components of the third-order alternating tensor. In writing numerical schemes, the discrete version of the scalar field $\phi(x, t)$ is represented by $\phi^{k}\left(x_{h}\right)$ representing the value of the function $\phi$ evaluated at the spatial location $x_{h}$ and at the $k^{\text {th }}$ discrete time level.

\section{$3 \quad$ Field Dislocation Mechanics}

Field Dislocation Mechanics [Ach10, Ach11, Ach01, Ach03, Ach04] is a pde-based model for understanding plasticity of solids as it arises from the nucleation, motion and interaction of defects in the elastic deformation of the material. It builds on the pioneering works of Kröner [Krö81], Mura [Mur63], Fox [Fox66], and Willis [Wil67] that almost exclusively develop the static elastic theory of continuously distributed dislocations, and extends this body of work to account for dissipative dislocation transport and non-linearity due to geometric and crystal elasticity effects. Preliminary thoughts and early efforts in modeling time-dependent dislocation dynamics within a pde framework are [WH74, CCHO97, CCV01]. More mature models are those of [WJCK01, SW03, RLBF03, XCSE03, Den04, AHLBM06] and the variational framework of [KCO02], none with the generality to deal with all three physical features of evolution of cores, nonlinear elasticity, and material inertia. Importantly, all of these models agree, implicitly at least, on the relationship between elastic incompatibility and the dislocation density given by $\operatorname{curl} \boldsymbol{U}^{e}=\boldsymbol{\alpha}$. This kinematic relationship implies an evolution statement for the total dislocation density tensor in the form of a conservation law for a vector-valued 2-form (and that is all) that geometrically constrains conversions of, for example, dislocations from one slip plane to another aided further by energetics and kinetics. At the scale of resolving individual dislocations, such an evolution statement coupled with the other laws of continuum mechanics, constitutive equations for the free energy density and a single dislocation velocity field is sufficient to generate a closed theory. In FDM, we work with exactly such a model. In the other models mentioned above the basic descriptors of dislocation fields take a variety of different forms including the number of such fields required, and a separate evolution statement for each such descriptor is prescribed for this varying collection of fields. Of course, all such statements have to be consistent with the 
fundamental conservation law for the total dislocation density mentioned above (which also implies, more-or-less, an evolution for the whole plastic distortion tensor), and it is not clear how this is attained in the various models.

In this paper, we largely work with the small deformation theory. The complete set of equations of FDM is

$$
\left.\begin{array}{l}
\boldsymbol{U}^{e}:=\operatorname{grad}(\boldsymbol{u}-\boldsymbol{z})+\boldsymbol{\chi} ; \quad \boldsymbol{U}^{p}:=\operatorname{grad} \boldsymbol{z}-\boldsymbol{\chi} \\
\operatorname{curl} \boldsymbol{\chi}=\boldsymbol{\alpha}=\operatorname{curl} \boldsymbol{U}^{e}=-\operatorname{curl} \boldsymbol{U}^{p} \quad \text { elastic incompatibility } \\
\operatorname{div} \boldsymbol{\chi}=0 \\
\operatorname{div}(\operatorname{grad} \dot{\boldsymbol{z}})=\operatorname{div}(\boldsymbol{\alpha} \times \boldsymbol{V}) \\
\operatorname{div}\left[\boldsymbol{T}\left(\boldsymbol{U}^{e}\right)\right]+\boldsymbol{f}=\rho \ddot{\boldsymbol{u}} \quad \text { balance of linear momentum } \\
\dot{\boldsymbol{\alpha}}=-\operatorname{curl}(\boldsymbol{\alpha} \times \boldsymbol{V}) \quad \text { conservation of Burgers vector content }
\end{array}\right\}
$$

The various fields are defined as follows. $\chi$ is the incompatible part of the elastic distortion tensor $\boldsymbol{U}^{e}, \boldsymbol{u}$ is the total displacement field, and $\boldsymbol{u}-\boldsymbol{z}$ is a vector field whose gradient is the compatible part of the elastic distortion tensor. $\boldsymbol{U}^{p}$ is the plastic distortion tensor. $\boldsymbol{\alpha}$ is the dislocation density tensor, and $\boldsymbol{V}$ is the dislocation velocity vector. $\boldsymbol{\alpha} \times \boldsymbol{V}$ (plastic strain rate with physical dimensions of time ${ }^{-1}$ ) represents the flow of Burgers vector carried by the dislocation density field moving with velocity $\boldsymbol{V}$ relative to the material. For the sake of intuition, indeed, when $\boldsymbol{\alpha}=\boldsymbol{b} \otimes \boldsymbol{l}$ with $\boldsymbol{b}$ perpendicular to $\boldsymbol{l}$ (an edge dislocation) and $\boldsymbol{V}$ in the plane spanned by $\boldsymbol{b}$ and $\boldsymbol{l}, \boldsymbol{\alpha} \times \boldsymbol{V}$ represents a simple shearing (strain rate) in the direction of $\boldsymbol{b}$ on planes normal to $\boldsymbol{l} \times \boldsymbol{V}$. The argument of the div operator in Eqs. $(3)_{5}$ is the (symmetric) stress tensor, $\boldsymbol{f}$ is the body force density, and the functions $\boldsymbol{V}, \boldsymbol{T}$ are constitutively specified. All the statements in Eqs. (3) are fundamental statements of kinematics or conservation. In particular, Eqs. (3) 6 is a purely geometric statement of conservation of Burgers vector content carried by a density of lines (see [Ach11] for a derivation) and Eqs. $(3)_{5}$ is the balance of linear momentum.

As for boundary conditions,

$$
\left.\begin{array}{l}
\boldsymbol{\chi} \boldsymbol{n}=0 \\
(\operatorname{grad} \dot{\boldsymbol{z}}-\boldsymbol{\alpha} \times \boldsymbol{V}) \boldsymbol{n}=0
\end{array}\right\} \text { on } \partial B
$$

are imposed along with standard conditions on displacement and/or traction.

The equations of FDM outlined above can be shown to imply a non-local continuum plasticity model whose stress response and plastic strain response are given as [Ach10]

$$
\begin{aligned}
& \boldsymbol{T}=\hat{\boldsymbol{T}}\left(\operatorname{grad} \boldsymbol{u}-\boldsymbol{U}^{p}\right) \\
& \dot{\boldsymbol{U}}^{p}=-\operatorname{curl} \boldsymbol{U}^{p} \times \hat{\boldsymbol{V}}\left(\boldsymbol{T}, \boldsymbol{U}^{p}, \text { curl } \boldsymbol{U}^{p}\right)
\end{aligned}
$$

where the constitutive functions $\hat{\boldsymbol{T}}, \hat{\boldsymbol{V}}$ are, in large part, guided by the structure of FDM. 


\section{2-D Straight edge dislocation model derived from FDM $^{1}$}

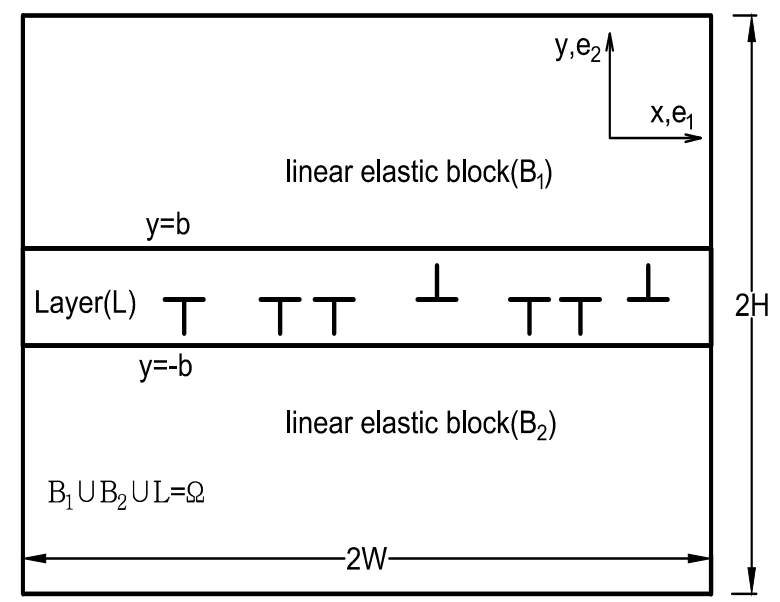

Figure 1: Geometry of the problem.

We consider the geometry shown in Fig. 1;

$$
\begin{aligned}
\Omega & =\{(x, y):(x, y) \in[-W,+W] \times[-H,+H]\}, \\
L & =\{(x, y):(x, y) \in[-W,+W] \times[-b,+b]\}, \\
0 & <b<H, \quad W>0 .
\end{aligned}
$$

In our notation we use $x \equiv x_{1}$ and $y \equiv x_{2}$, synonymously. The model may be viewed as a composite comprising two outer regions, $\Omega \backslash L$, whose stress response is purely linear elastic, and the layer $L$, of width $2 b$, whose response is elastic-plastic and where edge dislocations exist and FDM is active. The displacement fields $\boldsymbol{u}$ is continuous on the entire domain. We interpret the slip field in the layer as:

$$
\delta(x, t)=\int_{-b}^{+b} u_{1,2}(x, y, t) d y=u_{1}(x, b, t)-u_{1}(x,-b, t) .
$$

This field does not play an explicit role in the constitutive modeling, due to the latter's inherently bulk nature in our model, but we use it for discussing results related to tectonic rupture dynamics in Section 8.4. The dissipation on the whole body, defined as the difference of the rate of working of external forces and the rate of stored energy in the body, arises only from the layer (since everywhere else the body is elastic). Assume a stored energy density function of the form

$$
\psi\left(\boldsymbol{\epsilon}^{e}, \boldsymbol{\alpha}\right)+\eta\left(\boldsymbol{U}^{p}\right),
$$

\footnotetext{
${ }^{1}$ The content of this section with the exception of Sections 4.1 and 4.2 is excerpted from [AZ14] and included here for completeness.
} 
with stress given by $\boldsymbol{T}=\partial \psi / \partial \boldsymbol{\epsilon}^{e}$, where $\boldsymbol{\epsilon}^{e}$ is the symmetric part of the elastic distortion $\boldsymbol{U}^{e}$. The function $\psi$ is assumed to be positive-definite quadratic in $\boldsymbol{\epsilon}^{e}$ and the function $\eta$ is multiwell non-convex, endowing the energy function with barriers to slip and conferring preferred energetic status to certain plastic strains than others. Together, these two functions enable the robust modeling of overall total strain distributions in the layer displaying localized, smooth transitions between slipped and unslipped regions (or between the preferred strain states encoded in $\eta$ ). This crucially requires adding an energetic penalty to the development of high values of the dislocation density $\boldsymbol{\alpha}$, referred to as a core energy. In effect, the linear elastic stress and the core term tend to prevent a sharp discontinuity and the driving force from the non-convex $\eta$ term promotes the discontinuity, and it is the balance between these thermodynamic forces that sets the dislocation core width at equilibrium. Interestingly, it can be shown that while in the presence of just one component of plastic distortion only the linear elastic term suffices to give a finite core width (paralleling a fundamental result due to Peierls [Pei40]), with more than one component, the core regularization from the $\boldsymbol{\alpha}$ term is essential [AT11, LS08]. It is to be noted that the core energy is a fundamental physical ingredient of our model and not simply a mathematical regularization. In general, it is not expected to have the simple 'isotropic' form assumed here and, in fact, its characterization furnishes our model with a direct route of making contact with (sub)atomic physics [MPBO98, IRG15].

The dissipation in the model can be written as

$$
\begin{aligned}
\mathrm{D}= & \int_{L}\left(\boldsymbol{T}-\frac{\partial \eta}{\partial \boldsymbol{U}^{p}}\right): \dot{\boldsymbol{U}}^{p} d v+\int_{L} \frac{\partial \psi}{\partial \boldsymbol{\alpha}}: \operatorname{curl}(\boldsymbol{\alpha} \times \boldsymbol{V}) d v \\
= & \int_{L}\left(\boldsymbol{T}-\frac{\partial \eta}{\partial \boldsymbol{U}^{p}}\right):(\boldsymbol{\alpha} \times \boldsymbol{V}) d v+\int_{L} \operatorname{curl}\left(\frac{\partial \psi}{\partial \boldsymbol{\alpha}}\right): \boldsymbol{\alpha} \times \boldsymbol{V} d v \\
& +\int_{\partial L} \frac{\partial \psi}{\partial \boldsymbol{\alpha}}:(\boldsymbol{\alpha} \times \boldsymbol{V}) \times \boldsymbol{n} d a
\end{aligned}
$$

where $\boldsymbol{n}$ is the outward unit normal field to the body.

In the layer assume the ansatz

$$
\begin{aligned}
\boldsymbol{U}^{p}(x, y, t) & =U_{12}^{p}(x, y, t) e_{1} \otimes e_{2}+U_{22}^{p}(x, y, t) e_{2} \otimes e_{2} \\
& :=\phi(x, t) e_{1} \otimes e_{2}+\omega(x, t) e_{2} \otimes e_{2}
\end{aligned}
$$

where the functions $\phi(x, t), \omega(x, t)$ need to be defined.

Then

$$
\boldsymbol{\alpha}(x, y, t)=-\operatorname{curl} \boldsymbol{U}^{p}(x, y, t)=-\phi_{x}(x, t) e_{1} \otimes e_{3}-\omega_{x}(x, t) e_{2} \otimes e_{3}
$$

and

$$
\operatorname{curl} \boldsymbol{\alpha}(x, y, t)=\phi_{x x}(x, t) e_{1} \otimes e_{2}+\omega_{x x}(x, t) e_{2} \otimes e_{2} .
$$

In keeping with the 2-d nature of this analysis and the constraint posed by the layer on the dislocation velocity, we assume

$$
\boldsymbol{V}(x, y, t)=V_{1}(x, y, t) \boldsymbol{e}_{1}:=v(x, t) \boldsymbol{e}_{1}
$$


where $v(x, t)$ needs to be defined.

Note that with these assumptions, the boundary term in the dissipation vanishes for the horizontal portions of the layer boundary. We also assume

$$
\frac{\partial \psi}{\partial \boldsymbol{\alpha}}=\epsilon \boldsymbol{\alpha},
$$

where $\epsilon$ is a parameter with physical dimensions of stress $\times$ length $^{2}$ that introduces a length scale and essentially sets the width of the dislocation core, at equilibrium. For specific simplicity in this problem, we impose $\boldsymbol{\alpha}=\mathbf{0}$ on vertical portions of the layer boundary by imposing $\phi_{x}( \pm W, t)=\omega_{x}( \pm W, t)=0$.

With the above ansatz, the conservation law $\dot{\boldsymbol{\alpha}}=-\operatorname{curl}(\boldsymbol{\alpha} \times \boldsymbol{V})$ reduces to

$$
\begin{array}{lll}
\phi_{t}(x, t)=-\phi_{x} v(x, t) & \text { or } \quad & \hat{\alpha}_{1 t}=-\left(\hat{\alpha}_{1} v\right)_{x} \\
\omega_{t}(x, t)=-\omega_{x} v(x, t) & \text { or } \quad & \hat{\alpha}_{2 t}=-\left(\hat{\alpha}_{2} v\right)_{x},
\end{array}
$$

where $\hat{\alpha}_{1}:=-\phi_{x}=\alpha_{13}$ and $\hat{\alpha}_{2}:=-\omega_{x}=\alpha_{23}$. Equation 10 defines the evolution equations for the plastic distortion components $\phi, \omega$ once $v$ is defined as a function of $(x, t)$.

We now consider the dissipation

$$
\begin{aligned}
\mathrm{D} & =\int_{L} V_{1}\left\{e_{1 j 3}\left(T-A+\epsilon(\operatorname{curl} \boldsymbol{\alpha})^{T}\right)_{j r} \alpha_{r 3}\right\} d v \quad A_{j r}:=\left(\frac{\partial \eta}{\partial \boldsymbol{U}^{p}}\right)_{j r} \\
& =\int_{L} v(x, t)\left\{\begin{array}{r}
{\left[T_{12}(x, y, t)-A_{12}(x, t)+\epsilon \phi_{x x}(x, t)\right]\left(-\phi_{x}(x, t)\right)} \\
+\left[T_{22}(x, y, t)-A_{22}(x, t)+\epsilon \omega_{x x}(x, t)\right]\left(-\omega_{x}(x, t)\right)
\end{array}\right\} d v
\end{aligned}
$$

We make the choice

$$
\begin{aligned}
v(x, t):= & \frac{-1}{B_{m} l^{m-1}|\hat{\boldsymbol{\alpha}}|^{m}(x, t)}\left\{\begin{array}{r}
\phi_{x}(x, t)\left[\tau(x, t)-\tau^{b}(x, t)+\epsilon \phi_{x x}(x, t)\right] \\
+\omega_{x}(x, t)\left[\sigma(x, t)-\sigma^{b}(x, t)+\epsilon \omega_{x x}(x, t)\right]
\end{array}\right\} \\
& m=0,1 \text { or } 2 \\
\tau(x, t):= & \frac{1}{2 b} \int_{-b}^{b} T_{12}(x, y, t) d y ; \quad \tau^{b}:=A_{12} \\
\sigma(x, t):= & \frac{1}{2 b} \int_{-b}^{b} T_{22}(x, y, t) d y ; \quad \sigma^{b}:=A_{22}
\end{aligned}
$$

(i.e. kinetics in the direction of driving force [Ric71], in the context of crystal plasticity theory), where $\hat{B}=B_{m} l^{m-1}|\hat{\alpha}|^{m}$ is a non-negative drag coefficient that characterizes the energy dissipation by specifying how the dislocation velocity responds to the applied driving force locally and $l$ is an internal length scale, e.g. Burgers vector magnitude of crystals. For simplicity, we assume the drag to be a scalar but in general its inverse, the mobility, could be a positive-semidefinite tensor. In general, it is in $\hat{B}$ that one would like to model the effect of layer structural inhomogeneities impeding dislocations as well as the effect of other microscopic mechanisms of energy dissipation during dislocation motion.

The parameter $B_{m}$ is expected, in general, to be a function of $m$; however, for all values of $m, B_{m}$ has physical dimensions of stress $\times$ time $\times$ length $^{-1}$, and introduces another length scale related to kinetic effects. 
Then the dissipation becomes

$$
\begin{aligned}
\mathrm{D}= & \int_{L} \frac{1}{B_{m} l^{m-1}|\alpha|^{m}(x, t)}\left\{\begin{array}{r}
\phi_{x}(x, t)\left[\tau(x, t)-\tau^{b}(x, t)+\epsilon \phi_{x x}(x, t)\right] \\
+\omega_{x}(x, t)\left[\sigma(x, t)-\sigma^{b}(x, t)+\epsilon \omega_{x x}(x, t)\right]
\end{array}\right\}^{2} d x d y \\
& +\mathrm{R},
\end{aligned}
$$

where

$$
\mathrm{R}=\int_{x=-W}^{x=+W}-v(x, t)\left\{\begin{array}{c}
\phi_{x}(x, t) \int_{-b}^{b}\left[T_{12}(x, y, t)-\tau(x, t)\right] d y \\
+\omega_{x}(x, t) \int_{-b}^{b}\left[T_{22}(x, y, t)-\sigma(x, t)\right] d y
\end{array}\right\} d x .
$$

Recalling the definitions of the layer-averaged stresses $\tau, \sigma$ in (11), we observe that

$$
\mathrm{R}=0 \text { and } \mathrm{D} \geq 0 .
$$

To summarize, within the class of kinetic relations for dislocation velocity in terms of driving force, positive dissipation along with the (global) conservation of Burgers vector content governs the nonlinear and nonlocal slip dynamics of the model. Essentially, slip gradients induce stress and elastic energy and the evolution of the dislocation is a means for the media to relieve this energy, subject to conservation of mass, momentum, energy, and Burgers vector.

To further simplify matters, we make the assumption that $\omega \equiv 0$, i.e. no normal plastic strain in the composite layer. Suppressing the argument $(x, t)$, the governing equation for the plastic shear strain now becomes

$$
\phi_{t}=\frac{\left|\phi_{x}\right|^{2}}{B_{m} l^{m-1}|\alpha|^{m}}\left(\tau-\tau^{b}+\epsilon \phi_{x x}\right) .
$$

The parameter $m$ can be chosen to probe different types of behaviour. Especially, $m=0$ corresponds to the simplest possible (linear) kinetic assumption. Recall that

$$
\tau(x, t):=\frac{1}{2 b} \int_{-b}^{b} T_{12}(x, y, t) d y \quad \text { and } \quad \tau^{b}(x, t)=\frac{\partial \eta}{\partial \phi} .
$$

For the stored energy, we assume the form

$$
\frac{1}{2} \boldsymbol{\epsilon}^{e}: \boldsymbol{C} \boldsymbol{\epsilon}^{e}+\eta\left(\boldsymbol{U}^{p}\right)+\frac{1}{2} \epsilon|\boldsymbol{\alpha}|^{2},
$$

where $\boldsymbol{\epsilon}^{e}$ is the elastic strain tensor. The non-convex energy density function is chosen to be a multiple well potential, with the plastic shear strain values at its minima representing the preferred plastic strain levels. A typical candidate that we utilize in this paper is

$$
\eta=\frac{\mu \bar{\phi}^{2}}{\pi^{2}}\left(1-\cos \left(2 \pi \frac{\phi}{\bar{\phi}}\right)\right) .
$$

The displacement field in the model satisfies

$$
\rho \ddot{u}_{i}=T_{i j, j} \text { in } \Omega
$$


where, for an isotropic material,

$$
T_{i j}=\lambda \epsilon_{k k}^{e} \delta_{i j}+2 \mu \epsilon_{i j}^{e}
$$

$\lambda, \mu$ being the Lamé parameters and

$$
\begin{aligned}
E_{i j} & :=\frac{1}{2}\left(u_{i, j}+u_{j, i}\right) \\
\epsilon_{i j}^{e} & =E_{i j} \text { in the elastic blocks, i.e. } \Omega \backslash L \\
\epsilon_{12}^{e} & =\epsilon_{21}^{e}=E_{12}-\frac{\phi}{2} ; \quad \text { all other } \epsilon_{i j}^{e}=E_{i j} \text { in the fault layer } L,
\end{aligned}
$$

where $i, j$ take the values 1,2 . The governing equations of the system are thus

$$
\left\{\begin{array}{l}
\rho \frac{\partial^{2} u_{i}}{\partial t^{2}}=\frac{\partial T_{i j}}{\partial x_{j}} \text { in } \Omega \\
\frac{\partial \phi}{\partial t}=\frac{1}{B_{m} l^{m-1}}\left|\frac{\partial \phi}{\partial x_{1}}\right|^{2-m}\left(\tau-\tau^{b}+\epsilon \frac{\partial^{2} \phi}{\partial x_{1}^{2}}\right) \quad \text { in } L .
\end{array}\right.
$$

We make the choice $l=b$ (fault zone width in rupture dynamics; in crystals, a measure of the interatomic spacing). Then dimensional analysis suggests introducing the following dimensionless variables:

$$
\tilde{x}=\frac{x}{b}, \tilde{t}=\frac{V_{s} t}{b}, \tilde{u}=\frac{u}{b}, \tilde{T}=\frac{\boldsymbol{T}}{\mu}, \tilde{\tau^{b}}=\frac{\tau^{b}}{\mu}, \tilde{\epsilon}=\frac{\epsilon}{\mu b^{2}}, \quad \tilde{B}_{m}=\frac{V_{s}}{\mu / B_{m}}
$$

where $\mu$ is the shear modulus and $V_{s}=\sqrt{\mu / \rho}$ is the elastic shear wave speed of the material. The non-dimensional drag number $\tilde{B}_{m}$ represents the ratio of the elastic wave speed of the material to an intrinsic velocity scale of the layer material. The non-dimensionalized version of Eqs. (14) reads as:

$$
\left\{\begin{array}{l}
\frac{\partial^{2} \tilde{u}_{i}}{\partial \tilde{t}^{2}}=\frac{\partial \tilde{T}_{i j}}{\partial \tilde{x}_{j}} \quad \text { in } \Omega \\
\frac{\partial \phi}{\partial \tilde{t}}=\frac{1}{\tilde{B}_{m}}\left|\frac{\partial \phi}{\partial \tilde{x}_{1}}\right|^{2-m}\left(\tilde{\tau}-\tilde{\tau}^{b}+\tilde{\epsilon} \frac{\partial^{2} \phi}{\partial{\tilde{x_{1}}}^{2}}\right) \text { in } L .
\end{array}\right.
$$

The system (16) admits initial conditions on the displacement and velocity fields $\tilde{u}_{i}, \dot{\tilde{u}}_{i}$ and the plastic strain $\phi$. As mentioned before, we apply the Neumann condition $\phi_{x}=0$ on the left and right boundaries of the layer $L$ and for $\left(16_{1}\right)$ we utilize standard prescribed traction and/or displacement boundary conditions.

\section{1 $\tilde{B}_{m} \gg 1$ : Quasi-static, rate-dependent response}

We consider a generic, appropriately nondimensionalized, loading parameter (either applied traction or displacement b.c.s) that evolves as

$$
\frac{d \tau^{a}}{d \tilde{t}}=\Gamma
$$


where $\Gamma \ll 1$ is a dimensionless loading rate, assumed to be tunable to be as small as required. The restriction to monotonic loading is not essential, but will suffice for our purposes in this paper. We now introduce a slow time scale

$$
s=\frac{\tilde{t}}{\tilde{B}_{m}}
$$

and pose the governing system (16) in this slow time scale:

$$
\begin{aligned}
\frac{1}{\tilde{B}_{m}^{2}} \frac{\partial^{2} \tilde{u}_{i}}{\partial s^{2}} & =\frac{\partial \tilde{T}_{i j}}{\partial \tilde{x}_{j}} \\
\frac{\partial \phi}{\partial s} & =\left|\frac{\partial \phi}{\partial \tilde{x}_{1}}\right|^{2-m}\left(\tilde{\tau}-\tilde{\tau}^{b}+\tilde{\epsilon} \frac{\partial^{2} \phi}{\partial{\tilde{x_{1}}}^{2}}\right) \\
\frac{d \tau^{a}}{d s} & =\Gamma \tilde{B}_{m} .
\end{aligned}
$$

We note that $\tilde{B}_{m} \gg 1$, and require $\Gamma \leq \tilde{B}_{m}^{-1}$. Moreover, we assume evolutions restricted to $\frac{\partial^{2} \tilde{u}_{i}}{\partial s^{2}}=\mathcal{O}(1)$ (in the limit $\tilde{B}_{m} \rightarrow \infty$ ) to obtain the quasi-static system

$$
\begin{aligned}
0 & =\frac{\partial \tilde{T}_{i j}}{\partial \tilde{x}_{j}} \\
\frac{\partial \phi}{\partial s} & =\left|\frac{\partial \phi}{\partial \tilde{x}_{1}}\right|^{2-m}\left(\tilde{\tau}-\tilde{\tau}^{b}+\tilde{\epsilon} \frac{\partial^{2} \phi}{\partial{\tilde{x_{1}}}^{2}}\right) \\
\frac{d \tau^{a}}{d s} & =\mathcal{O}(1) .
\end{aligned}
$$

For $m=2,\left(19_{2}\right)$ has the form of a nonlocal Ginzburg-Landau (NGL) equation and for $m=1$, that of a nonlocal level set (NLS) equation. To our knowledge, the case $m=0$ corresponding to the simplest and most natural constitutive assumption for the dislocation velocity (i.e. a linear kinetic 'law') seems not to have been previously considered. We name it the nonlocal generalized Burgers (NGB) equation based on the following reasoning: when the coefficient of the first derivative term is a constant, the equation is indeed, up to a rescaling in time, the inviscid Burgers equation in Hamilton-Jacobi form. Of course, the coefficient is not a constant and contains a 'viscous regularization' that comes not as a uniform, linear parabolic term as in Burgers' original equation, but in a degenerate quasilinear parabolic form (see [AT11] for some implications), along with nonlocal and nonmonotone contributions. Regardless, for the lack of a better choice, the necessity of having a name to refer the equation by, and a desire to note the wonderful confluence of J. M. Burgers' contributions in fluid dynamics and crystal dislocations within our model, we christen the equation by the name we have adopted. Indeed, a distinguishing feature of Burgers equation is the modeling of shape-change of a wave profile with time-evolution and we find that it is this property of our NGB equation that allows it to predict a Peierls stress-like threshold for dislocation motion.

The quasi-static system (19) evolves on a time scale set by the drag coefficient under very slow or static loadings. Physically, we may expect this model to be of relevance to slipping in geomaterials and special situations in rupture dynamics [RCF04, CAS99], and polymeric composites under very slow loading rates. 


\section{2 $\tilde{B}_{m}<1$ : Quasi-static, rate-independent response}

This case is relevant to dislocation motion in crystalline materials under slow loadings. For dislocation motion well below the speed of sound, a typical value of the dislocation drag coefficient used in discrete dislocation methodology is $10^{-4} \mathrm{~Pa} \cdot s$ for Aluminum [KCC $\mathrm{KC}^{+}$. The ratio of the product of the magnitudes of the shear stress acting on a discrete dislocation and its Burgers vector to this parameter, say $B_{D D}$, is assumed to be the constitutive equation for the magnitude of the discrete dislocation's velocity. In order to estimate the magnitude of $B_{m}$ for our model corresponding to crystalline materials, we consider $(14)_{2}$ for $m=1$ and

observe that the coefficient of $\left|\frac{\partial \phi}{\partial x_{1}}\right|$ corresponds to the velocity of a slip front (whose derivative represents a dislocation herein) since, for a $\phi$ profile monotone increasing/decreasing in $x$, this is just the first-order wave equation (cf. [VBAF06]). In particular, if $\tau_{d}$ is a constant applied stress value and $b$ the Burgers vector magnitude,

$$
\frac{\tau_{d}}{B_{1}}=\frac{\tau_{d} b}{B_{D D}}
$$

which is just the statement of equality of speeds of dislocations in discrete dislocation methodology and our model, for $m=1$. Using the abovementioned value of $B_{D D}$ from $\left[\mathrm{KCC}^{+} 92\right]$, we obtain

$$
\tilde{B}_{1}=0.0297
$$

after non-dimensionalization according to (15). As mentioned earlier, $B_{m}$, for all values of $m$, has the same physical dimension as $B_{1}$ and in the following we assume them as having a common value except in one instance which we explicitly mention in Section 8.1. The non-dimensional, quasi-static systems analyzed herein $(19,22)$ do not require explicit consideration of the values of $B_{m}$.

We consider slow loading of the type (17) with $\Gamma \ll 1$, and a slow time scale of the form

$$
s=\Gamma \tilde{t} .
$$

On this time scale, the governing equations (16) take the form

$$
\begin{aligned}
\Gamma^{2} \frac{\partial^{2} \tilde{u}_{i}}{\partial s^{2}} & =\frac{\partial \tilde{T}_{i j}}{\partial \tilde{x}_{j}} \\
\Gamma \tilde{B}_{m} \frac{\partial \phi}{\partial s} & =\left|\frac{\partial \phi}{\partial \tilde{x}_{1}}\right|^{2-m}\left(\tilde{\tau}-\tilde{\tau}^{b}+\tilde{\epsilon} \frac{\partial^{2} \phi}{\partial \tilde{x}_{1}^{2}}\right) \\
\frac{d \tau^{a}}{d s} & =1 .
\end{aligned}
$$

Noting that $\Gamma \ll 1$ and $\tilde{B}_{m}<1$, we obtain the following quasi-static system:

$$
\begin{aligned}
0 & =\frac{\partial \tilde{T}_{i j}}{\partial \tilde{x}_{j}} \\
0 & =\left|\frac{\partial \phi}{\partial \tilde{x}_{1}}\right|^{2-m}\left(\tilde{\tau}-\tilde{\tau}^{b}+\tilde{\epsilon} \frac{\partial^{2} \phi}{\partial{\tilde{x_{1}}}^{2}}\right) \\
\frac{d \tau^{a}}{d s} & =1 .
\end{aligned}
$$


On time intervals in which (22) is an accurate approximation of the actual dynamics, the equivalent dynamics viewed on the fast time scale is (16), appended with

$$
\frac{d \tau^{a}}{d \tilde{t}} \approx 0
$$

We note an important fact related to the appropriateness of quasi-static systems like (22). Consider $\Upsilon$ and $\Phi$ as the $\tilde{u}_{i}(\cdot)$ and $\varphi(\cdot)$ fields on the body (viewed as functions of spatial coordinates alone) that satisfy the first two equations of (22) subject to boundary conditions for a particular value of the load $\tau^{a}$ (the load here can be thought of as a function on the boundary of the body). This can be stated abstractly as the fact that $\left(\Upsilon, \Phi, \tau^{a}\right)$ satisfy the functional equations

$$
F\left(\Upsilon, \Phi, \tau^{a}\right)=0
$$

Suppose now that the solution set of $\left(\Upsilon, \Phi, \tau^{a}\right)$-triples of the functional equation $F=$ 0 (the equilibrium set) admits connected one-dimensional paths. Let one such path be $\left(\Upsilon(s), \Phi(s), \tau^{a}(s)\right)$, where the function $\tau^{a}$ satisfies $\left(22_{3}\right)$. Then one can compute first and second partial derivatives with respect to $s$ of the fields $\tilde{u}_{i}$ and $\varphi$ corresponding to this 'equilibrium' path and, in general, these are not expected to vanish, even though the path belongs to the equilibrium set. However, due to the availability of the small parameters in (21), such a time-dependent 'solution' may be considered an appropriate approximate solution of the system (21). It is a remarkable fact that the full dynamics often does follow these equilibrium paths to a very good approximation. However, situations arise when states are reached along such paths where $\frac{d \tau^{a}}{d s}$ can no longer be linked uniquely to $\left(\frac{d \Upsilon}{d s}, \frac{d \Phi}{d s}\right)$. In these circumstances, the quasi-static system (22) provides no guidance on the actual evolution and only the full dynamics can decide whether jumps, on the slow time scale, between two equilibrium paths take place (if the $\tau^{a}$ corresponds to multiple states on the equilibrium set at the instant of the jump) or a single equilibrium path can be followed, or the equilibrium set is abandoned forever by the actual dynamics. At such instants, the time-derivatives in (21) become unbounded and (22) is no longer an appropriate representation of the dynamics (21). The time derivatives in the fast system (16) remain well-behaved, and it is this system that needs to be considered for accurate information on the actual dynamics.

When (22) is valid, $\left(\frac{d \Upsilon}{d s}, \frac{d \Phi}{d s}\right)$ associated with any state on an equilibrium path is related to $\frac{d \tau^{a}}{d s}$ through a linear operator that solely depends on the said state. This represents rateindependent response where the model has no internal time-scale and the evolution of fields depend on the rate of loading through a homogeneous function of degree 1.

\section{$5 \quad$ Numerical Schemes}

We gather the dimensionless governing equations in one place for convenience and then provide the numerical schemes for solving the equations:

$$
\left\{\begin{array}{l}
\frac{\partial^{2} u_{i}}{\partial t^{2}}=\frac{\partial T_{i j}}{\partial x_{j}} \quad \text { in } \Omega \\
\frac{\partial \phi}{\partial t}=\frac{1}{\tilde{B}_{m}}\left|\frac{\partial \phi}{\partial x_{1}}\right|^{2-m}\left(\tau-\tau^{b}+\epsilon \frac{\partial^{2} \phi}{\partial x_{1}^{2}}\right) \quad \text { in } L
\end{array}\right.
$$


where

$$
\begin{aligned}
& T_{i j}=C_{i j k l}\left(u_{k, l}-U_{k l}^{p}\right) \\
& C_{i j k l}=\lambda \delta_{i j} \delta_{k l}+\mu\left(\delta_{i k} \delta_{j l}+\delta_{i l} \delta_{j k}\right) \\
& \tau^{b}=\frac{2 \mu \bar{\phi}}{\pi} \sin \left(2 \pi \frac{\phi}{\bar{\phi}}\right) .
\end{aligned}
$$

Material properties are controlled by the Lamé constants $\lambda, \mu$ and the dimensionless drag coefficient $\tilde{B}_{m}$ together with the core energy $\epsilon \approx \mu b^{2}$. In general, the Finite Element Method $(\mathrm{FE})$ is used to solve the equation for balance of linear momentum in a staggered scheme that utilizes the plastic distortion $\boldsymbol{U}^{p}$ as a given quantity obtained by evolving $\boldsymbol{U}^{p}$ (or $\phi$ ) in the remaining part of the scheme. The general computing flow is shown in Fig. 2.

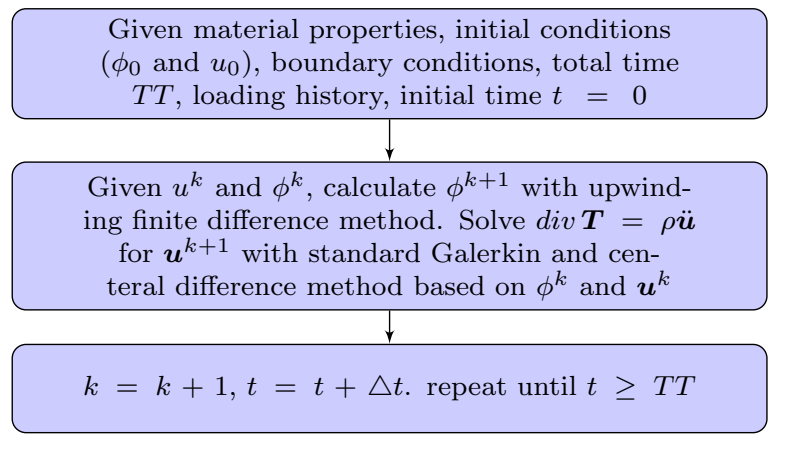

(a) dynamic equations

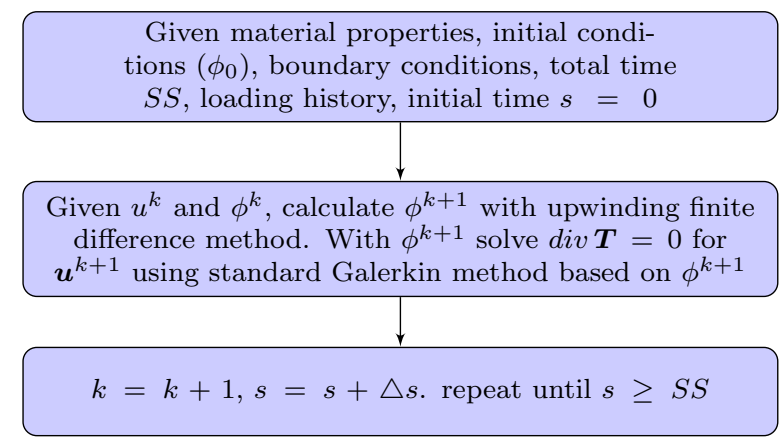

(b) quasi-static equations

Figure 2: Flow charts for dynamic (Eqs. (16)) and quasi-static (Eqs. (19)) models: $\phi$ and $\boldsymbol{u}$ are unkonwn plastic strain and displacement fields. $\boldsymbol{T}$ is Cauchy stress.

An FE mesh with an embedded 1-d finite difference grid is used. We use linear quadrilateral elements, with $5 \times 5$ Gauss quadrature points. Two types of FE meshes are created and used:

1. Mesh A: elements are of uniform size over the whole domain.

2. Mesh $B$ : elements are refined in and around the layer area.

Mesh A is used in Sec. 8 as it allows capturing stress wave propagation accurately over the whole body. Mesh B is used primarily to study the Peierls' stress problem, e.g., in Sec. 7.1.2 and 7.1.5. This is because we need a highly refined mesh in the layer to make statements independent of mesh size. We utilize regular quadrilateral elements of uniform size within the layer. Outside the layer, the size of the elements increases gradually as they get further from the layer. The layer is discretized up to 40 elements per Burgers vector, as required, while keeping the overall number of elements less than $150 \times 10^{3}$ (Fig. 3).

The 1-d, finite difference grid is embedded in the layer, coincident with the line $y=0$. Recall that the layer $L$ is always uniformly meshed (for both meshes A and B). Suppose that the layer is meshed into $M$ rows and $N$ columns, where $N$ is the total number of $1-\mathrm{d}$ grid points and $M$ is always an odd number so that the middle row of elements always have centres on $y=0$. Each column of FE elements in the layer correspond to exactly one grid 
point. Let $x_{k}$ be the $x$ coordinate of the $k^{t h} 1$-d grid point, which is at the center of the $k_{t h}$ element in the $(M+1) / 2$ row of layer elements. The value of $\boldsymbol{U}^{p}$ at each Gauss point within column $k$ is then set to be $\phi\left(x_{k}\right) \boldsymbol{e}_{1} \otimes \boldsymbol{e}_{2}$, where $\phi\left(x_{k}\right)$ is the value of $\phi$ evaluated at the $k^{\text {th }}$ grid point. Recall that the layer stress $\tau\left(x_{k}\right)$ is defined as $\frac{1}{2 b} \int_{-b}^{b} T_{12}(x, y, t) d y$. Let $T_{12}(I, k)$ denote the stress component $T_{12}$ at the $I^{\text {th }}$ Gauss point whose $x$ coordinate is $x_{k}$, and let $N_{k}$ be the total number of such Gauss points. Then $\tau\left(x_{k}\right)$ is calculated as

$$
\tau\left(x_{k}\right)=\frac{1}{N_{k}}\left(\sum_{I=1}^{N_{k}} T_{12}(I, k)\right) .
$$

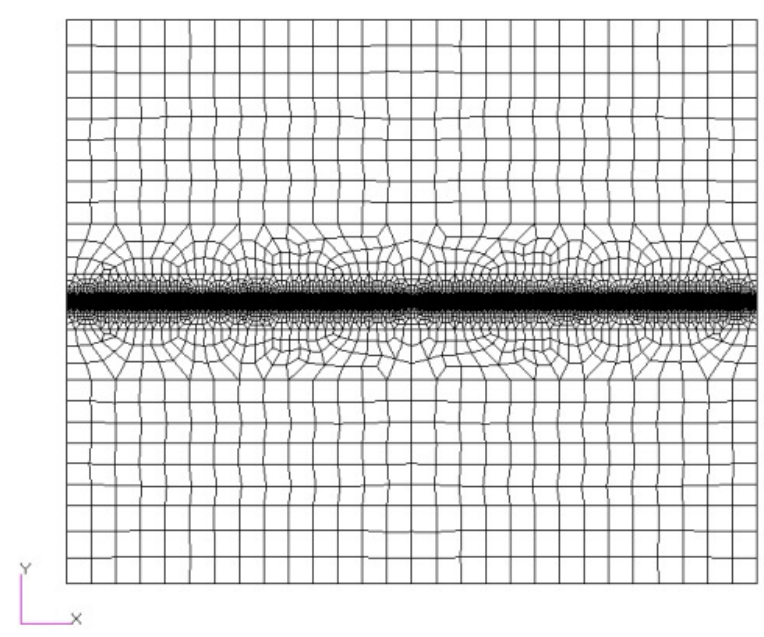

Figure 3: An example of FE mesh used in section 7.1. Elements are refined in and around the layer.

\subsection{Algorithm for evolution problems}

The numerical scheme developed in [DAZM13] is adopted and improved to solve $(23)_{2}$, the $\phi$ evolution $^{2}$. The basic idea is to infer the direction of wave propagation from the linearization of $(23)_{2}$ and use this direction in the actual nonlinear equation. Let $\triangle t$ be the time step and $\triangle h$ the spatial grid size of the finite difference grid. Due to the necessity of very small element sizes to demonstrate convergence, an explicit treatment of the diffusion term in $(23)_{2}$ becomes prohibitive because of a $\triangle t=\mathcal{O}\left(\triangle h^{2}\right)$ scaling. This is circumvented by treating the $\phi_{x x}$ term implicitly, resulting in a linearly implicit scheme as follows. We first linearize

\footnotetext{
${ }^{2}$ We thank Dr. Amit Das for his help regarding certain aspects of the implementation described in this Section.
} 
$(23)_{2}$ and discretize:

$$
\begin{aligned}
\delta \phi_{t}^{k}\left(x_{h}\right) & =-(2-m)\left(\frac{-\operatorname{sgn}\left(\phi_{x}^{k}\left(x_{h}\right)\right)}{\tilde{B}_{m}}\right)\left|\phi_{x}^{k}\left(x_{h}\right)\right|^{1-m}\left[\tau^{k}\left(x_{h}\right)+\epsilon \phi_{x x}^{k+1}\left(x_{h}\right)-\left(\tau^{b}\left(x_{h}\right)\right)^{k}\right] \delta \phi_{x}^{k}\left(x_{h}\right) \\
& +\frac{\left|\phi_{x}^{k}\left(x_{h}\right)\right|^{2-m}}{\tilde{B}_{m}}\left[\epsilon \delta \phi_{x x}^{k}\left(x_{h}\right)\right] \\
& +\frac{\left|\phi_{x}^{k}\left(x_{h}\right)\right|^{2-m}}{\tilde{B}_{m}}\left[\tau^{b^{\prime}}\left(x_{h}\right) \delta \phi^{k}\left(x_{h}\right)\right]
\end{aligned}
$$

where a quantity such as $\phi_{x}^{k}\left(x_{h}\right)$ implies the value of $\phi_{x}(x)$ evaluated at $h^{\text {th }}$ grid point at $k^{\text {th }}$ time step. The first term in (24) provides an advection equation with wave speed

$$
c^{k}\left(x_{h}\right)=(2-m)\left(\frac{-\operatorname{sgn}\left(\phi_{x}^{k}\left(x_{h}\right)\right)}{\tilde{B}_{m}}\right)\left|\phi_{x}^{k}\left(x_{h}\right)\right|^{1-m}\left[\tau^{k}\left(x_{h}\right)+\epsilon \phi_{x x}^{k+1}\left(x_{h}\right)-\left(\tau^{b}\left(x_{h}\right)\right)^{k}\right] .
$$

$\phi_{x}^{k}\left(x_{h}\right)$ and $\phi_{x x}^{k}\left(x_{h}\right)$ are obtained from central finite differences:

$$
\begin{aligned}
\phi_{x}^{k}\left(x_{h}\right) & =\frac{\phi^{k}\left(x_{h+1}\right)-\phi^{k}\left(x_{h-1}\right)}{2 \triangle h} \\
\phi_{x x}^{k}\left(x_{h}\right) & =\frac{\phi^{k}\left(x_{h+1}\right)-2 \phi^{k}\left(x_{h}\right)+\phi^{k}\left(x_{h-1}\right)}{\triangle h^{2}} .
\end{aligned}
$$

Based on the sign of $c_{k}, \phi_{x}^{k}$ is then computed by the following upwinding scheme:

$$
\phi_{x}^{k}= \begin{cases}\frac{\phi^{k}\left(x_{h+1}\right)-\phi^{k}\left(x_{h}\right)}{\triangle h} & \text { if } c^{k}\left(x_{h}\right)<0 \\ \frac{\phi^{k}\left(x_{h}\right)-\phi^{k}\left(x_{h-1}\right)}{\triangle h} & \text { if } c^{k}\left(x_{h}\right)>0 \\ \frac{\phi^{k}\left(x_{h+1}\right)-\phi^{k}\left(x_{h-1}\right)}{2 \triangle h} & \text { if } c^{k}\left(x_{h}\right)=0 .\end{cases}
$$

The time step is governed by a combination of a CFL condition and a criterion for stability for an explicit scheme for a linear ordinary differential equation:

$$
\triangle t^{k}=\min \left(\frac{\triangle h}{c^{k}\left(x_{h}\right)}, \frac{\tilde{B}_{m}}{\left|\phi_{x}^{k}\left(x_{h}\right)\right|^{2-m}\left(-\left(\tau^{b^{\prime}}\left(x_{h}\right)\right)^{k}\right.}\right) .
$$

Note that if $\phi_{x x}$ was evaluated at $k$, then the step size would also be bounded by $\frac{\Delta h^{2} \tilde{B}_{m}}{\epsilon\left|\phi_{x}^{k}\left(x_{h}\right)\right|}$, leading to a quadratic decrease in $\Delta t^{k}$ with element size. Treating $\phi_{x x}$ implicitly eliminates this constraint resulting in significant savings in computation time. $\phi_{h}^{k+1}$ is updated according to

$$
\begin{aligned}
& \frac{\phi^{k+1}\left(x_{h}\right)-\phi^{k}\left(x_{h}\right)}{\triangle t^{k}}=\frac{\left|\phi_{x}^{k}\left(x_{h}\right)\right|^{2-m}}{\tilde{B}_{m}}\left[\tau^{k}+\epsilon \phi_{x x}^{k+1}-\left(\tau^{b}\left(x_{h}\right)\right)^{k}\right] \\
\Rightarrow & \phi^{k+1}\left(x_{h}\right)-\epsilon \triangle t^{k} \frac{\left|\phi_{x}^{k}\left(x_{h}\right)\right|^{2-m}}{\tilde{B}_{m}} \phi_{x x}^{k+1}\left(x_{h}\right)=\phi^{k}\left(x_{h}\right)+\triangle t^{k} \frac{\left|\phi_{x}^{k}\left(x_{h}\right)\right|^{2-m}}{\tilde{B}_{m}}\left[\tau^{k}-\left(\tau^{b}\left(x_{h}\right)\right)^{k}\right] .
\end{aligned}
$$


The right hand side of the equation is known at current time $k$. But noting that $\phi_{x x}^{k+1}\left(x_{h}\right)$ is again computed from $\phi^{k+1}$ at $x_{h+1}, x_{h}$ and $x_{h-1}$, a system of linear equations of size $N$ has to be solved to get $\phi^{k+1}$. The computational expense of the linear solve is small compared to the savings obtained by relaxing $\triangle t^{k}$ corresponding to the explicit treatment of diffusion.

\subsection{Algorithm for equilibria}

In this section we record the derivation of a Quasi-Newton scheme for system (22), specifically the $\phi$ equation. In Sec. 6, we use this method to determine equilibrium states under zero or finite loads.

In the following, when we refer to $\phi_{I}$ we mean the discrete nodal list of values of the approximation to the function $\phi$ on a finite difference grid, corresponding to the $I^{\text {th }}$ iterate in the Quasi-Newton scheme. Consider the case $m=0$ (NGB) first. The residual for the $\phi$-equation is denoted by $F$ and defined as

$$
\left|\phi_{J x}^{i}\right|^{2}\left[\tau_{J}^{i}+\epsilon \phi_{J x x}^{i}-\frac{\partial \eta}{\partial \phi}\left(\phi_{J}^{i}\right)\right]=: F^{i}\left(\phi_{J}\right) .
$$

Here, $\tau_{J}^{i}$ is a function of $\phi_{J}$ only through $\phi_{J}^{i}$. The notation $(\cdot)_{J \ldots}^{i}$ denotes the value of the discrete approximation to the function $(\cdot) \ldots$ corresponding to the $J^{\text {th }}$ iterate for $\phi$ at the $i^{\text {th }}$ node. The second spatial derivative appearing in (29) is defined as in (25). For the first spatial derivative, the following scheme is used. Define

$$
c_{J}^{i}:=-2 \operatorname{sgn}\left(\phi_{J x}^{i}\right)\left|\phi_{J x}^{i}\right|\left[\tau_{J}^{i}+\epsilon \phi_{J x x}^{i}-\frac{\partial \eta}{\partial \phi}\left(\phi_{J}^{i}\right)\right],
$$

where both $\phi_{J x}^{i}$ and $\phi_{J x x}^{i}$ are evaluated from $\phi_{J}$ according to (25). With the value of the array $c_{J}$ in hand, $\phi_{J x}^{i}$ is redefined as

$$
\phi_{J x}^{i}= \begin{cases}\frac{\phi_{J}^{i+1}-\phi_{J}^{i}}{\triangle h}, & \text { if } c_{J}^{i}<0 \\ \frac{\phi_{J}^{i}-\phi_{J}^{i-1}}{\triangle h}, & \text { if } c_{J}^{i}>0 \\ \frac{\phi_{J}^{i+1}-\phi_{J}^{i-1}}{2 \triangle h}, & \text { if } c_{J}^{i}=0 .\end{cases}
$$

This array of values of $\phi_{J x}$ is then used in defining the residual (29).

The Newton-Raphson scheme obtained from the residual (29) is

$$
\begin{aligned}
-F^{i}\left(\phi_{J}\right) & =-c_{J}^{i} \delta \phi_{x}^{i}+\left|\phi_{J x}^{i}\right|^{2}\left[\mu \delta \phi^{i}+\epsilon \delta \phi_{x x}^{i}-\frac{\partial^{2} \eta}{\partial \phi^{2}}\left(\phi_{J}^{i}\right) \delta \phi^{i}\right] \\
\phi_{J+1}^{i} & =\phi_{J}^{i}+\delta \phi^{i},
\end{aligned}
$$


where the element $\delta \phi_{x}^{i}$ of the array of corrections $\delta \phi$ is defined as

$$
\delta \phi_{x}^{i}= \begin{cases}\frac{\delta \phi^{i+1}-\delta \phi^{i}}{\triangle h}, & \text { if } c_{J}^{i}<0 \\ \frac{\delta \phi^{i}-\delta \phi^{i-1}}{\triangle h}, & \text { if } c_{J}^{i}>0 \\ \frac{\delta \phi^{i+1}-\delta \phi^{i-1}}{2 \triangle h}, & \text { if } c_{J}^{i}=0\end{cases}
$$

This Newton-Raphson scheme leads to an asymmetric tridiagonal Jacobian matrix, which is also singular because the leading term $\phi_{J x}$ vanishes in dislocation free regions. To deal with that, we observe the residual also has a multiplier of $\left|\phi_{x}^{i}\right|$ and cancel it from both sides of the equation. This results in a Quasi-Newton method where the Jacobian matrix is modified. Of course, the residual is kept exactly in the form (29) without modification. Quasi-Newton iterations are continued until the $l^{\infty}$ norm of the residual, $|F|_{\infty}$, vanishes (up to a small tolerance).

For the NGL $(m=2)$ equation we use the exact Jacobian for the Newton-Raphson method given by

$$
-F^{i}\left(\phi_{J}\right)=\mu \delta \phi^{i}+\epsilon \delta \phi_{x x}^{i}-\frac{\partial^{2} \eta}{\partial \phi^{2}}\left(\phi_{J}^{i}\right) \delta \phi^{i} .
$$

For the NLS equation, we use a Quasi-Newton method based on the Jacobian matrix (34).

We follow a conventional nonlinear plasticity approach to solve the system (22). Fig. 4 shows the associated flow chart.

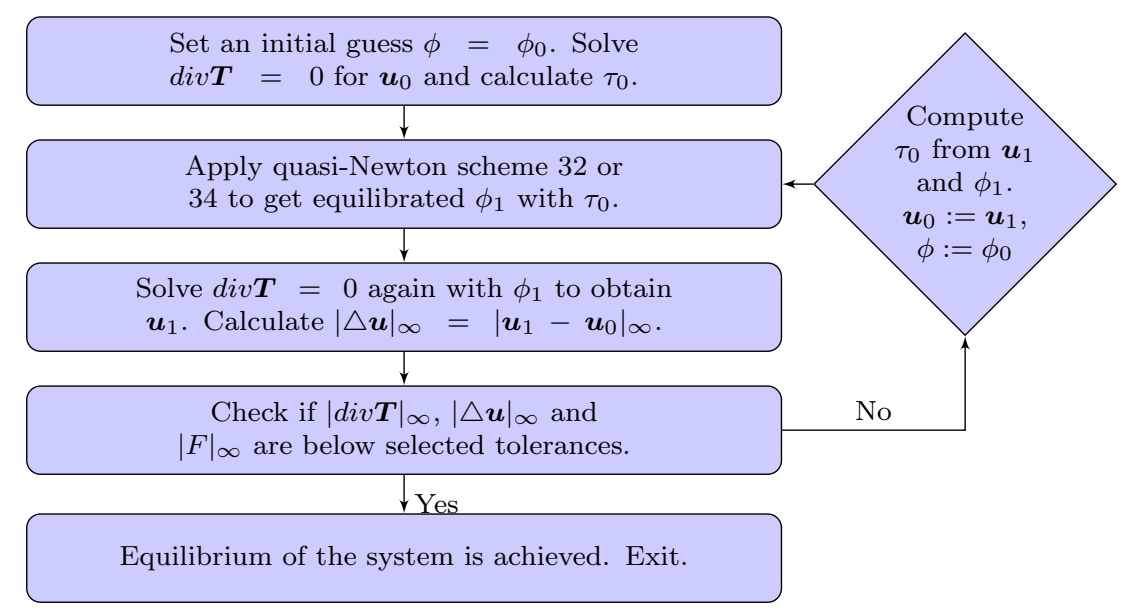

Figure 4: Flow chart for equilibrium problems: $\phi$ and $\boldsymbol{u}$ are unkonwn plastic strain and displacement fields. $\boldsymbol{T}$ is Cauchy stress. The initial guess $\phi_{0}$ is obtained from a pre-equilibrium solution. Note the repeated initialization of $\phi$ to $\phi_{0}$, which is found to be crucial for convergence.

For later reference, we record the definition of residuals used for determining system equilibria. For each finite difference node $i$ and a discrete function $\phi_{J}$, the residual $F^{i}\left(\phi_{J}\right)$ is defined through Eq. (29). The vector of FE, nodal displacement degrees of freedom is denoted by $\boldsymbol{u}$ and $\triangle \boldsymbol{u}$ is defined as the difference between two consecutive calculations as 
defined in Fig. 4. Both vectors $F$ and $\triangle \boldsymbol{u}$ are measured by their $l^{\infty}$ norm, i.e., suppose $N$ is the total number of nodes on the FE mesh (not including nodes on which Dirichlet boundary conditions are specified) and $M$ is the total number of finite difference grid points, then

$$
|\triangle \boldsymbol{u}|_{\infty}:=\max _{1 \leq i \leq 2 N}\left|u^{i}\right|, \quad|F|_{\infty}:=\max _{1 \leq i \leq M}\left|F^{i}\right|
$$

\section{Equilibrium Aspects}

We solve some key problems of classical dislocation theory [Nab87, HL82] in this Section, approached as equilibrium states of our dynamical model (16). While the classical theory involves singular dislocations with infinite-energy elastic fields (even on finite bodies), our solutions have finite energies and nonsingular cores. It is worth emphasizing that our equilibrium core distributions of dislocation density for a single dislocation are not a model assumption as in [Ach01, PLSG14, CAWB06]. These fields in our case, along with their corresponding non-singular stress distributions, correspond to equilibrium states of a dynamic theory where both the dislocation (core) distribution and the stress evolve to decrease the free energy of a body; the solutions in the aforementioned works, in particular the core distributions, have no such thermodynamic status. The larger implication of this feature is that FDM can serve as an idealized model for studying complex questions related to equilibrium and dynamic evolution (at realistic time-scales) of core structures of single and interacting dislocations under loads, utilizing input from finer length-scale models like Density Functional Theory [IRG15] and Molecular statics [Vit68, SW03, MPBO98] in defining its energetic constitutive ingredients (7), (12). Our model for $m=2$ (NGL), up to the definition of the layer stress $\tau$ and the use of the core energy, is essentially identical to that of the phase field model of dislocations [WL10, Den04].

\subsection{Equilibria of single edge dislocations}

The stress field of a single edge dislocation in an infinite domain is calculated, which is then validated by comparing with the closed-form classical solution for a single edge dislocation at the center of a finite cylindrical solid [HL82]

$$
\begin{aligned}
& \sigma_{11}=-D x_{2}\left(-\frac{3}{a^{2}}-\frac{2 x_{2}^{2}}{\left(x_{1}^{2}+x_{2}^{2}\right)^{2}}+\frac{3}{x_{1}^{2}+x_{2}^{2}}\right) \\
& \sigma_{22}=-D x_{2}\left(-\frac{1}{a^{2}}-\frac{2 x_{1}^{2}}{\left(x_{1}^{2}+x_{2}^{2}\right)^{2}}+\frac{1}{x_{1}^{2}+x_{2}^{2}}\right) \\
& \sigma_{12}=D x_{1}\left(-\frac{1}{a^{2}}-\frac{2 x_{2}^{2}}{\left(x_{1}^{2}+x_{2}^{2}\right)^{2}}+\frac{1}{x_{1}^{2}+x_{2}^{2}}\right)
\end{aligned}
$$

where $D=\mu b / 2 \pi(1-\nu)$ with $\nu$ the Poisson's ratio, and $a$ is the radius of the cylinder (assumed to be $\infty$ here). $x_{1}, x_{2}$ are the in-plane coordinates measured from the center of the dislocation.

We solve for the stress field of a single dislocation in an infinite domain under no applied loads by utilizing a 2-D body of finite size and applying traction boundary conditions 
according to the analytical stress field. Specifically, we compute the analytical stress $\boldsymbol{\sigma}^{*}$ of boundary points according to Eqs. (36) and then apply a boundary traction $\boldsymbol{t}=\boldsymbol{\sigma}^{*} \boldsymbol{n}$, where $\boldsymbol{n}$ is the outward unit normal to the boundary. The rigid deformation of the body is removed by fixing $u_{1}$ and $u_{2}$ at the corner $(-W,-H)$ as well as fixing $u_{2}$ at $(W,-H)$.

We are interested in obtaining special equilibria of the system (16) corresponding to the field of a single dislocation. Because of the degenerate and nonlinear nature of the equilibrium equations for $m=0,1$, approaching the question by directly trying to approximate equilibria is a formidable task. Instead, evolution to equilibrium could be a desirable route. However, the time scale of evolution of (16) is extremely restrictive and since equilibrium states are the only items of concern here, the question could as well be approached by evolving the quasi-static dynamics (19) from suitably close initial conditions. There is a complication in that the system (19) belongs to a class in which simpler versions [CP89, DAZM13] exhibit extremely sluggish dynamics out of states which, nevertheless, are known not to be equilibria. Thus, we adopt the following approach:

1. We consider all $m=0$ (NGB), $m=1$ (NLS) and $m=2$ (NGL) models. The initial condition on $\phi$ is a hyperbolic tangent function whose first spatial derivative gives the initial distribution of the dislocation density according to (9) representing a single dislocation:

$$
\phi(x, t=0)=\frac{1}{2}(\bar{\phi} \tanh (a x)+\bar{\phi}),
$$

where we choose $\bar{\phi}=0.5$, and $a=\sqrt{\mu / 4 \epsilon}$. By the definition of $\boldsymbol{\alpha}$, the initial Burgers vector magnitude $b_{0}$ may be approximated as $b_{0}=\int_{L} \int_{d}-\phi_{x}(x, 0) \boldsymbol{e}_{3} d y d x \approx-2 \bar{\phi} b=$ $-b$.

2. The dynamics (19) is evolved to get to a state that satisfies approximate equilibrium conditions up to certain numerical tolerances. We conservatively specify a threshold value of $\left|\phi_{s}\right|_{\infty}<5 \times 10^{-5}$, where $\left|\phi_{s}\right|_{\infty}$ represents the $l^{\infty}$ norm of the discrete $\phi$ field, i.e. $\left|\phi_{s}\right|_{\infty}:=\max _{1 \leq i \leq N}\left|\phi_{s}^{i}\right|,(N$ being the total number of finite difference nodes.). This threshold is conservative because the profile-change of the dislocation field becomes indiscernible to the eye long before $\left|\phi_{s}\right|_{\infty}$ gets to this value.

We refer to these practically static states as dislocation pre-equilibria.

3. We use the NGL, NLS, NGB dislocation pre-equilibrium states as initial guesses to solve the corresponding nonlinear equilibrium equations of (16). The numerical implementation is described in Section 5. Dimensionless tolerances required by the scheme to determine whether an equilibrium state is achieved are chosen as follows:

$$
|\triangle u|_{\infty}<2 \times 10^{-4},|F|_{\infty}<5 \times 10^{-10}
$$

Recall that $|F|_{\infty}$ measures the residual of the $\phi$ equilibrium equation (and therefore our tolerance requires equilibria to be at least 5 orders of magnitude slower than dislocation pre-equilibria); $|\triangle u|_{\infty}$ measures the residual of the displacement fields between two consecutive approximations; $|\operatorname{div} T|_{\infty}$ tests mechanical force balance, which is always resolved on the scale of $10^{-15}$. 
We refer to the attained solutions as (unloaded) $N G L / N L S / N G B$ dislocation equilibria. Furthermore, in what follows, we need the following definitions:

- Equilibria of the NGB dynamics are sought, closest to an NGL dislocation preequilibria in the sense of the latter serving as an initial guess for the procedure outlined above (i.e. list item 3). We refer to such an equilibrium state as an $N G L-s-N G B$ dislocation equilibrium (the ' $s$ ' stands for 'start').

- NGB equilibrium states are sought, as defined above, but now under the action of a nonzero applied traction on the body. We refer to such a state as a loaded $N G B$ dislocation equilibrium.

In this section, mesh $\mathrm{B}$ is primarily used except for the calculations reported in Fig. 6 and 7 (recall that we have two types of FE meshes: mesh A has uniformly refined elements over the whole body; mesh B is refined only in and around the layer). All required simulation details are grouped in Table 1.

\begin{tabular}{|l|l|}
\hline simulation parameters & values \\
\hline domain $(W \times H)$ (mesh A) & $110 b \times 110 b$ \\
domain $(W \times H)$ (mesh B) & $110 b \times 90 b$ \\
layer element $(h)$ (mesh A) & $0.3 b \sim 2 b$ \\
layer element $(h)$ (mesh B) & $0.1 b$ \\
core-energy strength $(\epsilon)$ & 1 \\
Young's modulus $(E)$ & $70 G P a$ \\
shear modulus $(\mu)$ & $26 G P a$ \\
Burgers vector $(b)$ & $4.05 \times 10^{-10} m$ \\
Layer thickness $(d)$ & $2 b$ \\
\hline
\end{tabular}

Table 1: Simulation details.

Fig. 5 shows the dislocation profiles of NGL, NLS and NGB (unloaded) dislocation equilibria starting from the initial condition (37).

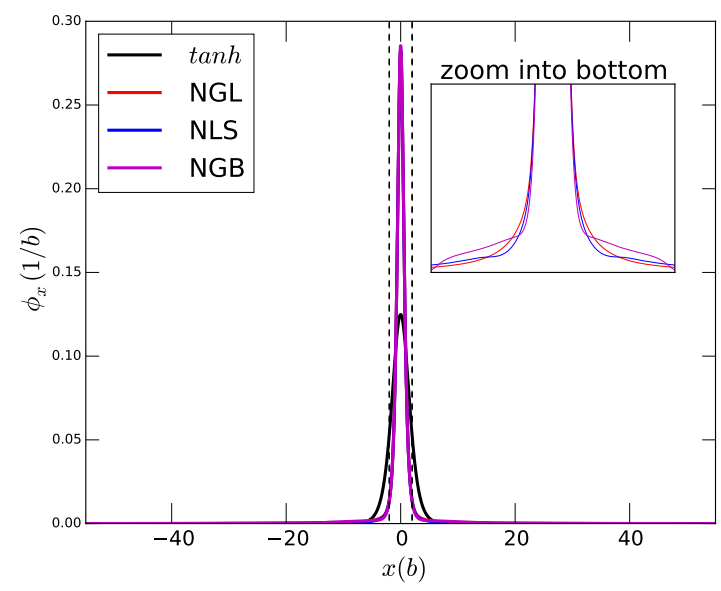

Figure 5: The equilibrated dislocation profiles. The dotted lines denote the dislocation core. 
The quantity $\tau$ is of primary interest in this section as it is analogous to $\sigma_{12}$ of Eq. (36) with $x_{2}=0$. Figures 6, 7(a) and 7(b) together demonstrate that the numerical stress field obtained from our model is quantitatively comparable to that from classical solutions. In particular, Fig. 6 shows that the stress field does not strictly rely on a highly refined mesh, i.e., a mesh as coarse as $h=2 b$ can still provide a stress result consistent with the classical solution outside the dislocation core. As shown in Fig. 6, the difference between the equilibrated averaged layer stress $\tau$ (blue) and the analytical solution along $x$ axis (cyan) is indiscernible beyond the dislocation core (marked by the two dotted lines).

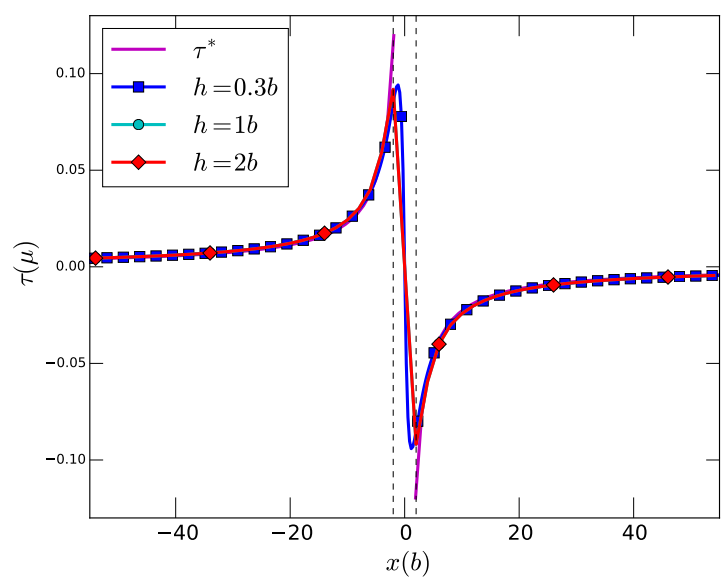

Figure 6: Comparison of $\tau$ with the analytical solution at $x=0$. The dotted lines denote the dislocation core. $\tau^{*}$ is a closed-form $T_{12}$ on $x_{1}$ axis from the classic method. $h$ is the finite element size in the layer, measured with Burgers vector.

Fig. 7(a) shows the contour of shear stress $\sigma_{12}$ on the body. The difference between the numerical and the closed-form classical solution is quantified by calculating an error measure $E R$ defined by

$$
E R_{i j}(x, y)=\frac{\left|\sigma_{i j}^{*}(x, y)-\sigma_{i j}(x, y)\right|}{\left|\sigma_{i j}^{*}(x, y)\right|},
$$

where $\sigma_{i j}^{*}$ and $\sigma_{i j}$ are the solutions from (36) and numerical computation, respectively. At the lines $x=0$ and $x= \pm y$ where the denominator $\left|\sigma_{i j}^{*}\right|$ vanishes, $E R_{12}$ values are not plotted. The maximum value of $\sigma_{12}$ along these 'blank' regions given by our model is $5.7 \times 10^{-5} \mu$ which is achieved on the boundaries of the dislocation core. Some other data points along the lines are: $4.6573 \times 10^{-6} \mu$ at $(30 b, 30 b)$ and $1.4360 \times 10^{-5} \mu$ at $(50 b, 50 b)$. To sum up, it can be concluded that the error $\left(E R_{12}\right)$ is primarily restricted to the core area; the overall patterns and values are in close agreement. $E R_{12}$ reaches up to around $40 \%$ at the core boundaries due to the (unphysical) singularity of the analytical solution. Similar comparisons are obtained for other stress components for both the NLS and NGB cases. We think of such dislocated states in an unloaded body as stressed metastable states. 


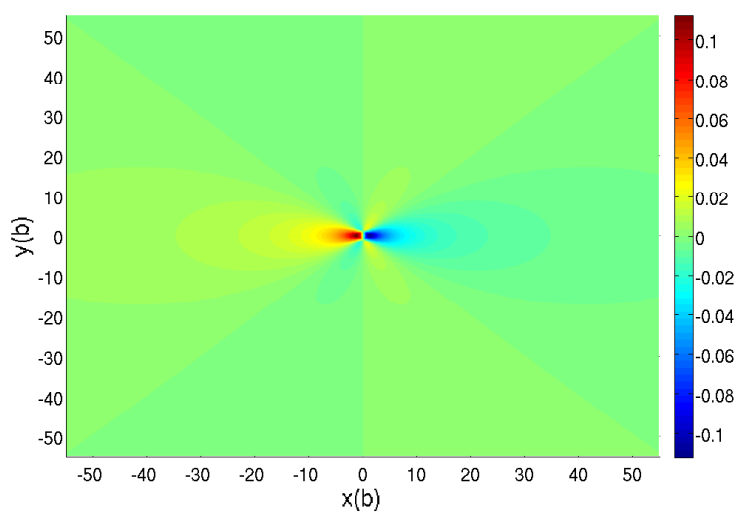

(a) Equilibrated FDM stress field $\sigma_{12}$ of the edge dislocation in an infinite media.

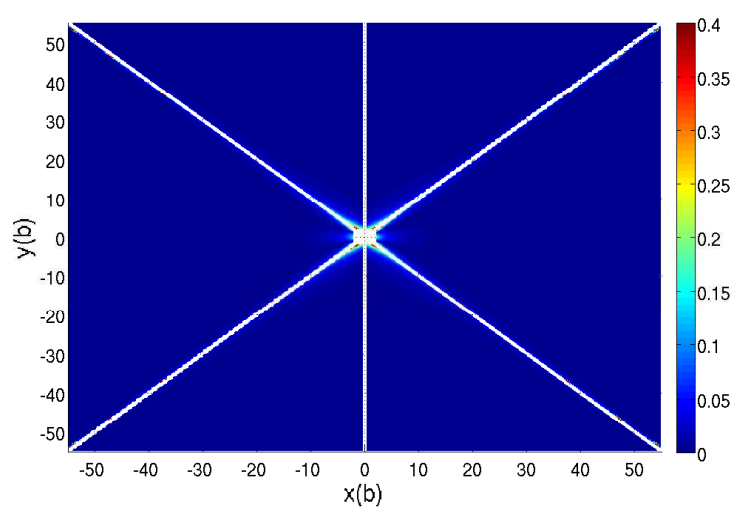

(b) $E R_{12}:$ a measure of difference between FDM results and the analytical results outside the core.

Figure 7: Comparison of numerical stress with analytical solution.

Two important observations on unloaded dislocation equilibria are:

- The (unloaded) NGL dislocation equilibrium is found to be identical to the NGL-sNGB dislocation equilibrium. The former is also an equilibrium state for the NLS dynamics. These are verifications for our numerical procedures as it is easy to see that an equilibrium state for the NGL dynamics must be so for both the NLS and NGB dynamics.

- We find that the shapes of the NGB dislocation equilibrium (obtained from the NGB dislocation pre-equilibria) and the NGL-S-NGB dislocation equilibrium are different. One needs to zoom into the bottom of Figure 5 to appreciate this difference, which is shown in Fig. 8. Apparently, the NGB dislocation equilibrium leads to a profile with curved steps on both sides of the core while the NGL-S-NGB dislocation equilibrium has a smooth profile with no steps.

This difference of shape will be further discussed in the following Section as it produces completely different solutions for loaded problems. 


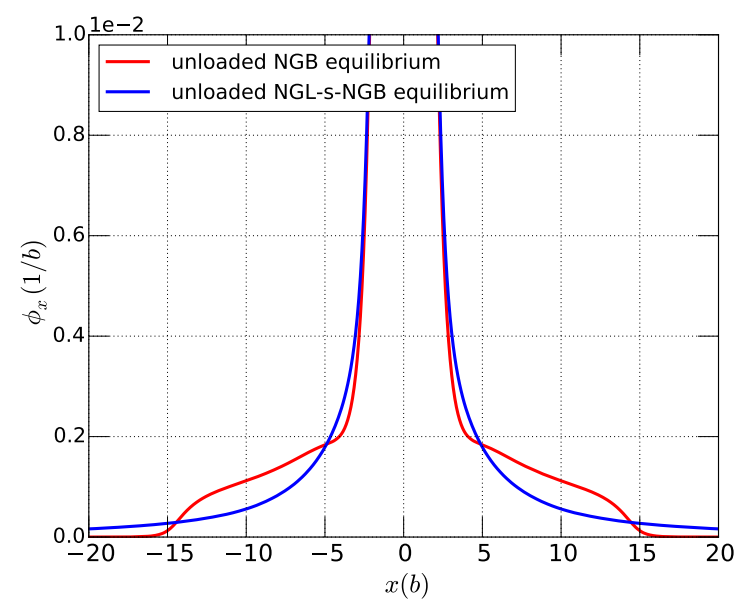

Figure 8: Comparison of two equilibria from different initial guesses: NGB equilibrium takes NGB pre-equilibrium as initial guess. NGL-s-NGB equilibrium takes NGL pre-equilibrium.

\subsubsection{Dislocation equilibria under load}

We apply a simple shear traction boundary condition, where the traction vector is defined by,

$$
\boldsymbol{t}=\tau^{a}\left(n_{2} \boldsymbol{e}_{1} \otimes n_{1} \boldsymbol{e}_{2}\right)
$$

$\boldsymbol{n}=n_{1} \boldsymbol{e}_{1} \otimes n_{2} \boldsymbol{e}_{2}$ is the outer normal of the surfaces.

We first choose $\tau^{a}=5 \times 10^{-5} \mu$ and seek equilibrium solutions of (14). The initial guess for our quasi-Newton iteration method is taken to be the unloaded NGB dislocation equilibrium. The system achieves equilibrium, up to the numerical tolerance specified by (38). More importantly, the equilibrated dislocation stays in the original position. This is shown in Fig. 9. The equilibrated core profile under load is slightly different from the zero load dislocation equilibrium, especially at the bottom. But the observation that the dislocation is not displaced is sufficient to demonstrate that the system (22) allows a dislocation equilibrium under a small but finite shear load.

We also make the following observations:

1. Up to $\tau^{a}=6.5 \times 10^{-5} \mu$, the system can still get equilibrated. The dislocation profile is close to that of $\tau^{a}=5 \times 10^{-5} \mu$, demonstrated in Fig. 10 .

2. When $\tau^{a}>6.5 \times 10^{-5} \mu$, our numerical scheme cannot converge below the specified tolerance. Specifically, when $\tau^{a}>10^{-4} \mu$, the residuals $|\Delta u|_{\infty}$ blow up quickly (the residuals are defined in Eq. 35).

3. The attainment of loaded dislocation equilibria is sensitive to the initial guess. In particular, we cannot obtain a dislocation equilibrium solution if the unloaded NGLs-NGB dislocation equilibrium of Fig. 8 is adopted as the initial guess.

4. The NGL and NLS systems cannot attain dislocation equilibria (using our computational strategy) starting from their no-load dislocation equilibria. We have tested this hypothesis down to applied loads of $5 \times 10^{-8} \mu$. This also serves as a partial verification 
of our numerical procedures since it can be shown that a no-load single dislocation equilibrium profile in an infinite body for the NLS dynamics has to move as a rigid traveling wave with uniform speed under arbitrary, non-zero applied loads.

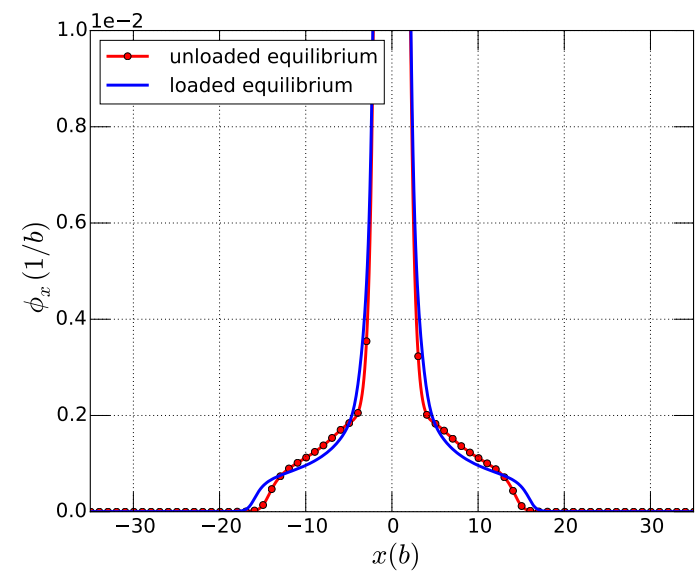

Figure 9: Equilibrium for load $5 \times 10^{-5} \mu$, compared to unloaded $N G B$ dislocation equilibrium.

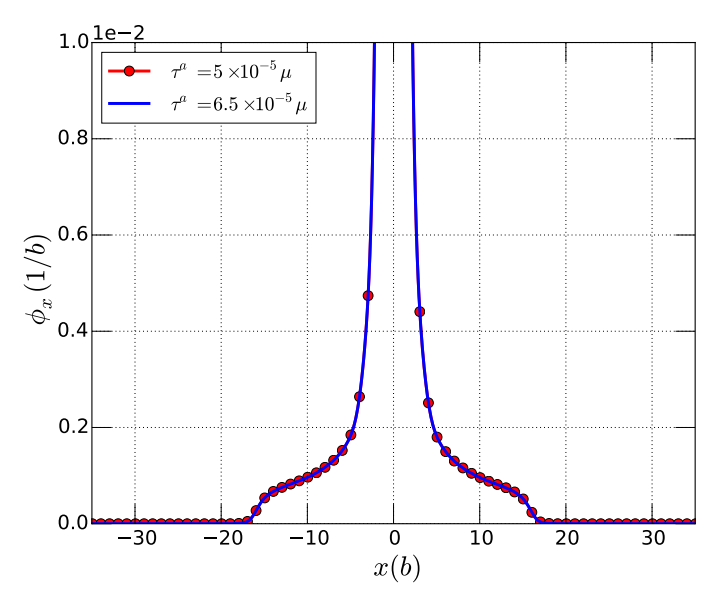

Figure 10: Equilibria for loads $5 \times 10^{-5} \mu$ and $6.5 \times 10^{-5} \mu$ are on top of each other.

In order to better understand the difference between the NGB and NGL models with respect to attainment of equilibrium under load, we analyze and plot the two constituent parts of their residuals: the energetic driving force term $\left(\tau+\epsilon \phi_{x x}-\tau^{b}\right)$ and the leading transport term $\left(\phi_{x}^{2}\right.$ of NGB, 1 of NGL). The energetic driving force terms are shown in Fig. 11(a) and 11(b). Specifically, the NGB case corresponds to a loaded equilibrium state of $\tau^{a}=5 \times 10^{-5} \mu$. Since the NGL dynamics cannot sustain a loaded equilibrium, we consider one particular state during its quasi-static evolution according to (19) under the same constant applied load. An immediate observation is as follows. Even though the energetic driving force for the NGB model is much greater in magnitude outside the core than its NGL counterpart, its 'transport multiplier,' $\phi_{x}^{2}$, essentially vanishes beyond $[-3.5 b, 3.5 b]$; within $[-3.5 b, 3.5 b]$, the NGB energetic driving force happens to be extremely close to zero (as shown in the inset of Fig. 11(b)). To the contrary, NGL has an all-positive energetic driving force after load is applied, with especially large values in the core area, and NGL does not have any leading transport term to counterbalance this effect and stop dislocation motion. 


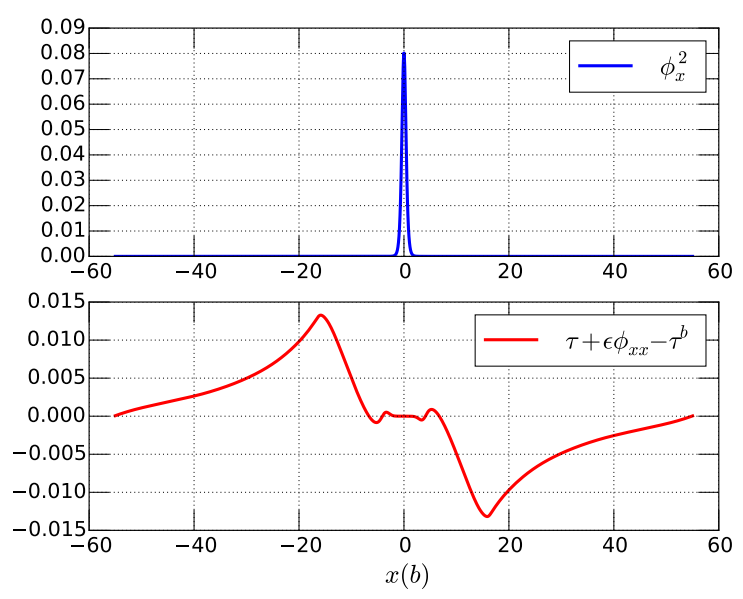

(a) NGB

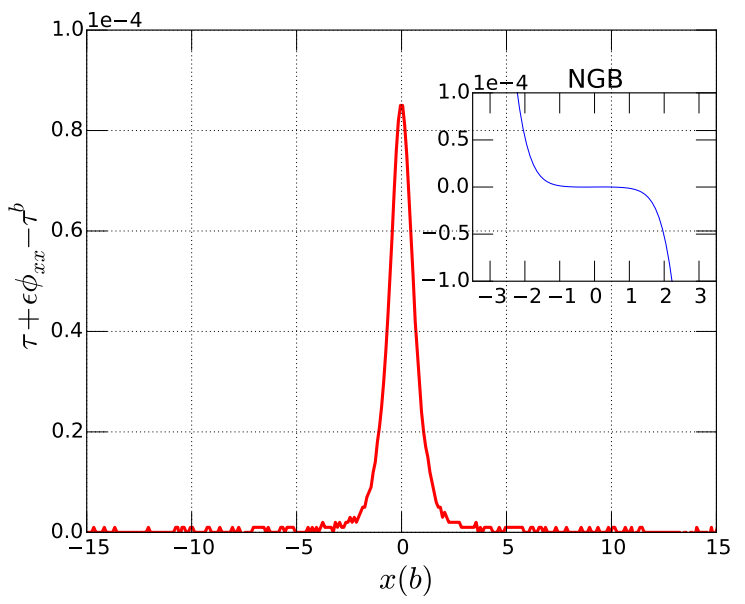

(b) NGL

Figure 11: Comparison of equilibrium and motion of $N G B$ and $N G L$ single dislocations under load $5 \times 10^{-5} \mu$.

Thus, the attainment of NGB equilibria under load is not simply a matter of getting the energetics of a model right but delicately dependent on the form of the dynamics, which in this case follows from the conservation of Burgers vector on dislocation density evolution. Said another way, equilibria in dynamic models need not necessarily be a consequence of energetics alone.

\subsection{The failure of Linear Elasticity in sustaining a compact core}

Figures 12 shows the inadequacy of the use of just linear elasticity, i.e. without any nonconvexity in the stored energy function, in producing an equilibrium dislocation with a compact core (we use the word 'compact' here to simply mean 'spatially localized'). Dislocation (pre-) equilibria of (19) are sought under no applied load, now with $\eta \equiv 0$ and $\epsilon=0$ in (12), so that the stored energy function simply contains the linear elastic term. All three dynamics start from the same tanh function (37). Although mechanical equilibrium (i.e. force balance) is satisfied at each time step of the dynamics (19), the dislocation density field is unable to sustain a compact core and spreads out thinly over the domain (the Burgers vector vector content has to be conserved with the Neumann boundary conditions (on $\phi_{x}$ ) in force). 


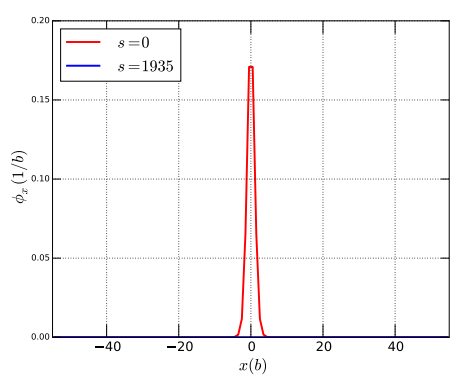

(a) $m=2$

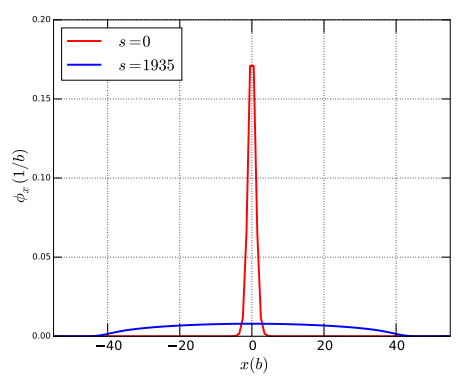

(b) $m=1$

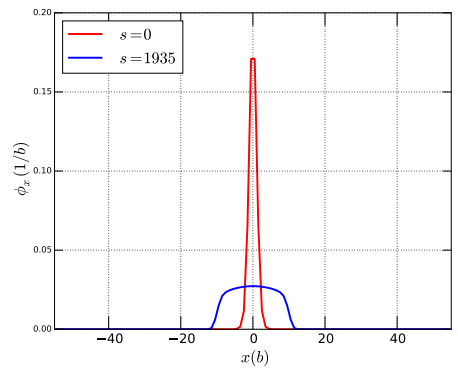

(c) $m=0$

Figure 12: Initial and final state of dislocation profiles with linear elasticity. Neumann condition $\phi_{x}=0$ is imposed on both ends of $\phi$. All three dynamics cannot sustain compact dislocation cores. $N G L$ becomes flat. The dislocations of $N L S$ and NGB keep flattening but with decreasing $\left|\phi_{s}\right|_{\infty}$. $\left|\phi_{s}\right|_{\infty}<10^{-5}$ for both $N L S$ and $N G B$ at $s=1935$.

The above example makes it clear why the existence of an equilibrium dislocation cannot be a prediction of the classical linear elastic theory of dislocations and the Discrete Dislocation methodology based on it $^{3}$, when coupled to any notion of energy minimization, whether global or local; there is no reason for an unloaded linear elastic body to sustain an energy concentration in it, unless the fact is enforced by an extraneous hard constraint.

Nabarro in his book [Nab87] remarks that "The theory of continuously distributed dislocations in a medium obeying Hooke's law and the theory of isolated dislocations having Burgers vector of the order of the interatomic spacing in a crystal are not always equivalent approaches to the same limit.." He further observes that "The attempt to build up a dislocation theory while neglecting the non-Hookean forces which hold a single dislocation together and prevents its thinly spreading over the glide plane is bound to encounter difficulties similar to those of the 'purely' electromagnetic theory of the electron. In this theory it is impossible to reconcile the electrostatic and electromagnetic estimates of the mass, because no allowance has been made for the mass associated with the non-Coulombian forces which bind together a cloud of charge, all of negative sign."

While we agree with Nabarro's assessment of the failure of the use of linear elasticity theory alone to address the problem at hand, we feel, and show in this paper, that the continuously distributed setting is ideally suited to account for relevant nonlinearities of dislocation mechanics and is a fundamentally sound approach to the theory of isolated dislocations with Burgers vector of the order of the interatomic spacing, including their dynamics, where the classical theory of isolated singular dislocations is quite inadequate.

\subsection{Equilibrium of dislocation Pile-ups}

This section demonstrates the approach for solving dislocation pile-up problems within FDM. Computationally, solving a problem involving an array of dislocations (including collections

${ }^{3}$ To be clear, it is of course not a goal of Discrete Dislocation methodology to answer questions related to the existence of a dislocation in any sort of thermodynamic equilibrium. 
with positive and negative dislocations) is essentially the same as solving a single dislocation problem, except for a change of the initial condition on the field $\phi$.

A key classical problem of the theory of dislocations is the following. A set of dislocations of identical sign lie on a slip plane. The set of dislocations pile up against obstacles, usually grain boundaries, under applied shear stress. What is the equilibrated state of the dislocations under the combination of their mutually repulsive interactions and the applied load? A mathematical model for this problem was developed and solved by Eshelby, Frank and Nabarro [EFN51] using classical dislocation theory. We refer to this model as the 'classical model,' and summarize the essential elements of [EFN51] relevant for our purposes. The classical model solves the following force equilibrium equations:

$$
\sum_{i=1, i \neq j}^{n} \frac{A}{x_{j}-x_{i}}+P\left(x_{j}\right)=0, j=1,2, \ldots . n,
$$

where $P(x)$ is the applied stress at the point $x$, and $x_{j}$ are the equilibrium positions of the dislocations. $A$ is a stress unit depending on the dislocation type. For an edge dislocation, $A=\mu b / 2 \pi(1-v) . A=1$ is chosen in the following derivation for convenience. Let $x_{i}$ be the roots of the polynomial

$$
f=\prod_{i=1}^{n}\left(x-x_{i}\right)
$$

and it is then realized that the logarithmic derivative of $f(x)$ is the stress of $x$ due to all dislocations, i.e.

$$
\frac{f^{\prime}}{f}=\sum_{i=1}^{n} \frac{1}{x-x_{i}}
$$

where $f^{\prime}:=\frac{d f}{d x}$. The stress at $x$ with the $j_{t h}$ dislocation missing is

$$
\frac{f^{\prime}}{f}-\frac{1}{x-x_{j}}
$$

The value of this expression at $x=x_{j}$ is obtained by taking the limit

$$
\lim _{x \rightarrow x_{j}} \frac{\left(x-x_{j}\right) f^{\prime}(x)-f(x)}{\left(x-x_{j}\right) f(x)}=\frac{1}{2} \frac{f^{\prime \prime}\left(x_{j}\right)}{f^{\prime}\left(x_{j}\right)} .
$$

Equation (41) can then be reformulated as

$$
\left.\begin{array}{l}
f\left(x_{j}\right)=0 \\
\frac{1}{2} \frac{f^{\prime \prime}\left(x_{j}\right)}{f^{\prime}\left(x_{j}\right)}+P\left(x_{j}\right)=0
\end{array}\right\} j=1,2, \ldots n
$$

To solve (46), Eshelby, Frank, and Nabarro ingeniously consider the equation

$$
f^{\prime \prime}(x)+2 P(x) f^{\prime}(x)+q(n, x) f(x)=0,
$$


noting that if $q(n, x)$ can be chosen such that (47) has an $n^{\text {th }}$ degree polynomial solution $f^{*}$ whose roots are real and distinct (with $q$ non-singular at the roots), then $f^{*}$ is a solution to (46) with the roots of $f^{*}$ being equilibrated dislocation positions along the 1-d slip-plane.

We study two pile-up problems within our 'layer model' that have been analytically solved in [EFN51]. Namely, find the equilibrium positions of

1. a row of $n$ dislocations under zero applied load, the outer two being locked;

2. the outer two dislocations in the row locked, with the array under an applied shear load.

For the purpose of generating closed-form results, a strategy for dealing with locked dislocations, effectively transforming them into applied loads, is described in [EFN51]. We solve these problems using exactly the same approach as we solve for the equilibrium of a single dislocation.

\subsubsection{Pile-up without load}

Consider five dislocations in a traction free body, i.e. $n=5$ in Eq. (41). The two dislocations at the ends of the array are pinned (by setting the velocity within the pinned dislocation cores to be zero).

\begin{tabular}{|l|l|}
\hline parameter name & value \\
\hline domain width $(W)$ & $110 b$ \\
domain height $(H)$ & $90 b$ \\
No. of elements & 16320 \\
core-energy strength $(\epsilon)$ & 0.25 \\
Young's modulus $(E)$ & $70 G p a$ \\
shear modulus $(\mu)$ & $26 G p a$ \\
Burgers vector $(b)$ & $4.05 \times 10^{-10} \mathrm{~m}$ \\
\hline
\end{tabular}

Table 2: simulation details for pile-up simulations

We solve this problem with the NLS $(m=1)$ model without loss of generality (the same results are obtained with the NGL and NGB models). The initial condition for $\phi$ is a superposition of spatially translated 'piecewise tanh' functions so that the dislocation spikes occur at $x=\{-40,-10,0,10,40\}$. The simulation details are grouped into Table 2. A multiple well $\eta$ function is essential for modelling a scenario with all dislocations of the same sign.

Even without any load, the dislocated body cannot be in a metastable equilibrium for an arbitrary initial configuration. This is due to the strong repulsive interactions between the dislocations in the pile-up. The dislocations (only the middle three dislocations can move freely) tend to re-distribute in the slip plane to achieve equilibrium. We plot the final dislocation distribution and the stress field. Specifically, Fig. 13 shows the initial and equilibrated configuration of the dislocations. The dotted lines indicate the positions predicted by the classical method. We see that the second and fourth dislocations (from the 
left) move towards the boundary while the dislocation in the middle is motionless. Fig. 14 shows the corresponding equilibrated stress field.
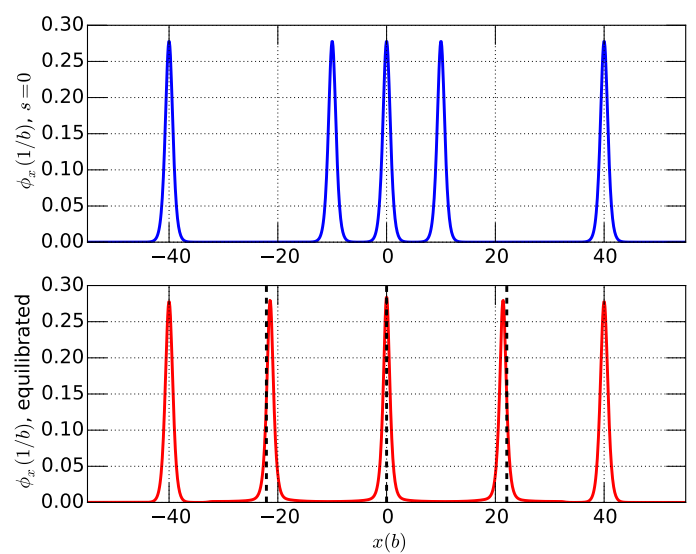

Figure 13: Pile-up motions. top: initial condition; bottom: equilibrated states; result from closed-form solution marked by black dotted lines.

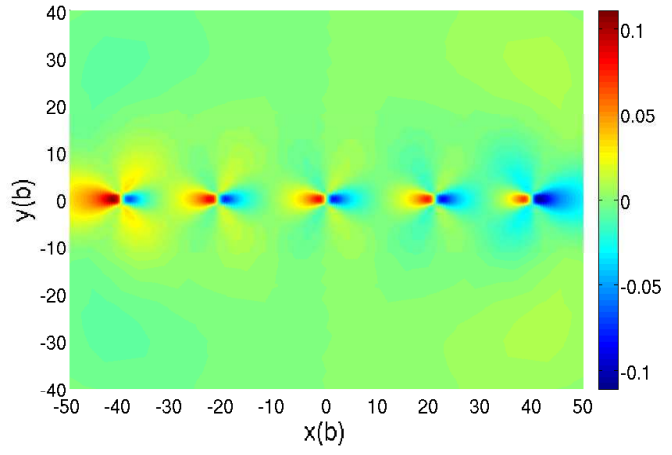

Figure 14: Unloaded $T_{12}$ of equilibrated pileup.

\subsubsection{Loaded pile-up}

An equilibrium, piled-up array of dislocations under shear load is simulated. We consider $n=5$ in Eq. (41) with the outer two dislocations pinned, which is again modeled by requiring the dislocation velocity to vanish within the corresponding dislocation cores. A traction boundary condition defined by Eq. (40) is applied with $\tau^{a}=0.05 \mu$. The positions of the dislocations in the array are arbitrarily initialized. Fig. 15 shows the initial and equilibrated configurations. The applied load makes the dislocations pile up against the left pinned dislocation. The equilibrium configuration displays a stress concentration against the left boundary. Fig. 16 shows the equilibrated shear stress field. 

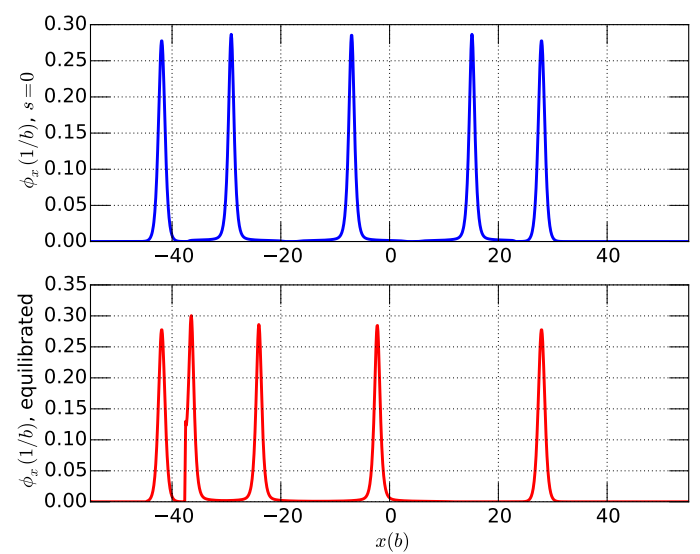

Figure 15: Pile-up under shear. top: initial condition; bottom: equilibrated states.

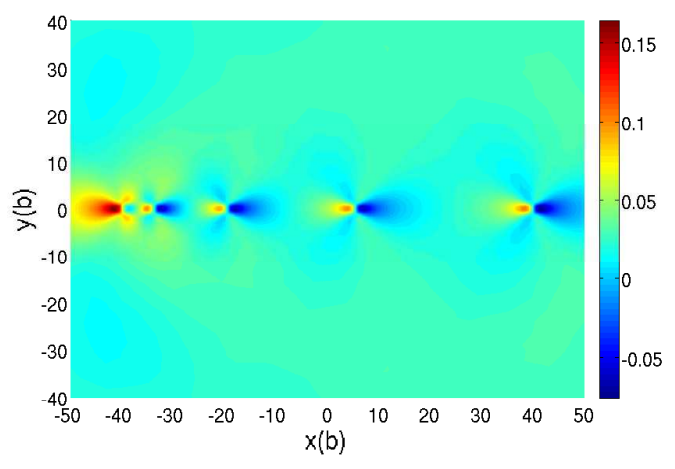

Figure 16: $T_{12}$ of equilibrated pile-ups under shear load.

\section{Dislocation motion in quasi-static deformations}

In this section, we utilize our computational methodology to study the models described in Sections (4.2) and (4.1). The context is phenomena related to dislocation motion when the material deformation may be nominally assumed as quasi-static.

\subsection{Peierls Stress in Continuum Mechanics?}

Applying a shear stress to a body sets an otherwise equilibrated dislocation under no load in motion. The relation between the magnitude of the applied stress and the dislocation velocity, as predicted by our models, is studied in this section, with particular emphasis on exploring the question of whether a Peierls stress can exist within our models of dislocation dynamics. The Peierls stress is the applied stress required to move a dislocation, and the question of its theoretical determination was first investigated by Peierls [Pei40] and improved by Nabarro [Nab47]. The improved model has since been called the Peierls model or the Peierls-Nabarro model ${ }^{4}$. Since then, this has been a vast area of study with increasingly sophisticated models: at the continuum level with some notion of discreteness [LH59, Sch99, MBW98, Pic02], atomistic level with interatomic potentials [BJC $\left.{ }^{+} 01, \mathrm{BCBK} 06, \mathrm{KBC} 12\right]$ and atomistic level with DFT input [LKBK00]. Such studies have focused on energetic aspects since the problem intrinsically corresponds to very slow to vanishing rates of loading at macroscopic time scales. To our knowledge, the current state of the art of Molecular Dynamics simulations is not capable of effectively probing the possible slow time scale dynamics that may be in play in this problem.

In the Peierls model, the existence of the Peierls stress arises from the change in total energy induced by a change in position of a dislocation (one assumes bodies of large enough

\footnotetext{
${ }^{4}$ Peierls also formulated and answered the question of the equilibrium profile of a non-singular, continuously distributed dislocation density field representative of a single isolated dislocation under no load in an infinite body.
} 
extent). In this sense, the Peierls stress is expected to vanish in any continuum mechanical model where the system energy is translationally-invariant, i.e., a dislocation is always in 'neutral equilibrium' before the application of load, and will move under any (small) perturbation.

However, the Peierls model does not include the possibility of a moving dislocation profile changing its shape, as it treats a dislocation as a rigid object (mathematically, a traveling wave) during motion. Clearly, if shape changes do occur on the application of load then, even by simply the logic of the Peierls model, it seems natural that the system energy can change even at the onset of motion and thus have an effect on the question of existence of a Peierls stress in continuum models. This was our hypothesis, essentially based on energetic arguments, in studying the question within our continuum model. However, as we report in Fig. 11, the matter is not simply dependent on energetics but also critically on the form of the evolution equation for $\phi$ that is a consequence of incorporating consideration of the conservation of Burgers vector on evolution.

We emphasize that the purpose of our study is not to deny the classical explanation of the Peierls stress arising from lattice discreteness; instead, it is to explore features of a theoretical model of plausible material behavior in a systematic way and, in so doing, hopefully uncovering possible complementary mechanisms for the physical phenomenon.

Two important physical observations are in order here:

- An order-of-magnitude to keep in mind in this Section is that the expected Peierls stress in FCC single crystals (a reasonable class of materials for comparison of our results) is $\sim 1 M P a$; translated in terms of the shear modulus, $\mu$, of $26 G P a$ adopted in our work, this amounts to $\sim 4 \times 10^{-5} \mu$.

- The Peierls stress question is unequivocally a question of evolution, albeit extremely slow (which in a sense makes the problem rather difficult to address by procedures that attempt to resolve atomic vibrations). Thus, strictly, simply demonstrating a loaded and an unloaded single dislocation equilibria does not suffice, as it does not address the question of whether the loaded equilibrium is dynamically accessible from the unloaded equilibrium under the specified loading history. A quasi-static approximation may be adopted, but the equilibrium trajectory then needs to be justified as being acceptable, as discussed in Sec. 7.1.2.

The common simulation details used in this Section are listed in Table 3.

\begin{tabular}{|l|l|}
\hline parameter name & value \\
\hline domain width $(W)$ & $110 b$ \\
domain height $(H)$ & $90 b$ \\
No. of elements & 16320 \\
core-energy strength $(\epsilon)$ & 0.25 \\
Young's modulus $(E)$ & $70 G P a$ \\
shear modulus $(\mu)$ & $26 G P a$ \\
Burgers vector $(b)$ & $4.05 \times 10^{-10} \mathrm{~m}$ \\
\hline
\end{tabular}

Table 3: Simulation details for Peierls stress problems. 


\subsubsection{Discrete mesh as surrogate for lattice discreteness effects}

An apparent Peierls stress effect may be demonstrated within our model by making the finite element mesh a part of the physical model as a crude model of some effects of a discrete lattice, and thus not asking questions of convergence with respect to mesh refinement. The following example demonstrates the idea, using the NLS $(m=1)$ dynamics. The domain is of size $[110 b \times 70 b]$ uniformly meshed by $251 \times 35$ quadrilateral elements. The corresponding 1 -d finite difference grid element size of $\phi$ is then $0.44 b$. In addition, the core-energy strength $\epsilon$ is set to be 0.1 .

The other simulation details are kept the same as in Table. 3. The initial condition on $\phi$ is an NLS dislocation pre-equilibria as defined in Section 6. The loading corresponds to a traction staircase with respect to time, as shown in the bottom panel of Fig. 17. The dislocation motion is monitored by plotting $\phi_{x}$ with time. The dislocation core is of finite size but we define the location of the dislocation as the $x$-coordinate of the peak of the $\phi_{x}$ profile at any instant of time. Since $\phi$ evolves in the form of a moving wave front, we can measure the speed of the dislocation by defining an average velocity $\bar{V}$,

$$
\bar{V}=\frac{\bar{X}}{\bar{T}},
$$

i.e., a prescribed distance $\bar{X}$ that the dislocation travels divided by the required time $\bar{T} . \phi_{s}$ is also of primary interest here as it indicates the evolution of the system on the slow time scale $s$; as usual, we measure $\phi_{s}$ by its $l^{\infty}$ norm.

The result in Fig. 17 shows an apparent Peierls stress effect: when the applied load is held at the magnitudes of $0.06 \mu$ and $0.11 \mu$, the dislocation remains motionless. In particular, $\left|\phi_{s}\right|_{\infty}$ grows a little when the load is changed from 0 to $0.06 \mu$ and from $0.06 \mu$ to $0.11 \mu$, but then quickly drops to zero. The average dislocation speed remains zero. Once the load is increased from $0.11 \mu$ to $0.16 \mu$, the dislocation moves significantly with velocity $0.2 V_{s}$. Note that $\left|\phi_{s}\right|_{\infty}$ begins to oscillate around the value of 0.06 and does not drop to zero as earlier. The result suggests that there exists an apparent Peierls stress in between the value of $0.11 \mu$ and $0.16 \mu$. A more precise value can be found by subdividing the load increments.

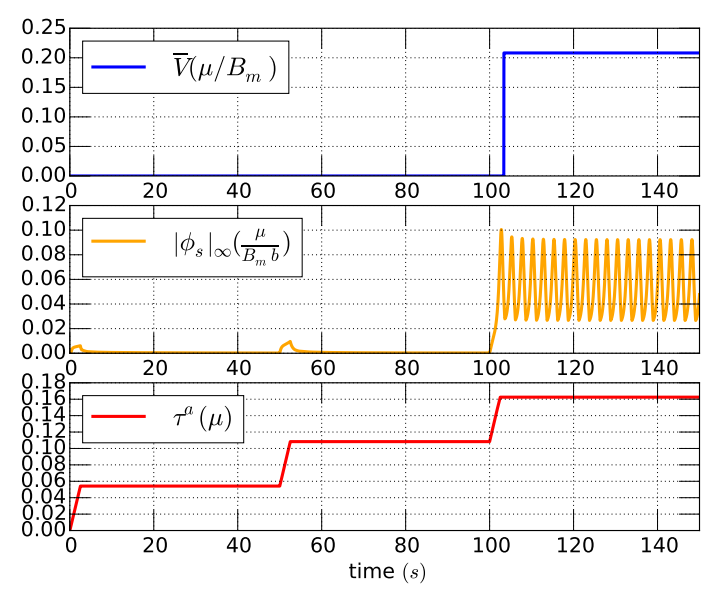

Figure 17: Peierls stress effect of a coarse mesh. top: the average velocity of the dislocation. middle: $\left|\phi_{s}\right|_{\infty}$. bottom: history profile of applied shear stress. 
The value of the stress threshold is completely unrealistic (too high). Moreover, the apparent Peierls stress effect is only valid for this particular discrete mesh, and decreases in a more refined mesh. As a practical device, an optimal mesh size could be associated with a target Peierls stress level in mind, in exploring problems where features of dislocation mechanics apart from the determination of Peierls stress is the subject of study.

Of course, our overriding goal in this paper is to explore the behavior of solutions to our pde model via numerical approximation. To this end, we strive to make statements independent of the mesh as demonstrated in Section 7.3.

\subsubsection{Peierls stress effects for small $B_{m}$ : the case of crystal dislocations}

As already shown in Sections 6.1 and 6.1.1, the NGB model allows not only an unloaded dislocation equilibrium, but also a dislocation equilibrium with finite shear load up to $6.5 \times$ $10^{-5} \mu$. More importantly, the equilibrated dislocation is not moved by the shear load, but deformed from its unloaded dislocation equilibrium shape.

Those solutions are obtained by solving for equilibria of (16). In particular, for the loaded dislocation equilibrium profile, we start with an initial guess corresponding to the NGB dislocation equilibrium (under no load) and use the Quasi-Newton method to obtain the loaded dislocation equilibrium solution, up to the tolerance specified in Eq. (38). Computationally, this procedure can be interpreted as the appropriate discrete analog of finding solutions to the quasi-static system (22), i.e. following a trajectory on the equilibrium set, parametrized by the applied load. We note here that this general philosophy is also adopted in lattice statics explorations of the Peierls stress. In computing these results, we use mesh B where the layer elements are of size $0.1 \mathrm{~b}$. Additionally, the results are verified by convergence tests as discussed later in Sec. 7.3.

Thus, we conclude that our NGB dynamics displays a Peierls stress effect, subject to checking that no untoward instability is observed on running the fast-scale dynamics (16) out of the initial state corresponding to the unloaded equilibrium dislocation when subjected to load.

We want to check whether a small but finite load like $5 \times 10^{-5} \mu$ displaces the dislocation when inertia is involved. As dislocation motion is an inherently dissipative process, it is important that the loading mode for Peierls stress-related questions be such that external energy supply is available as the dislocation moves/evolves; applied, constant-in-time traction loadings allow for this possibility and this is what we utilize in this Section.

Specifically, all simulations are performed in the following way. Initially, we obtain a state $\left(\phi_{0}, \boldsymbol{u}_{0}\right)$ that satisfies equilibrium conditions for (16). $\phi_{0}$ and $\boldsymbol{u}_{0}$ are then used as initial conditions of $\phi$ and $\boldsymbol{u}$ for Eqs. (16). A constant traction boundary condition defined by Eq. (40) with $\tau^{a}=5 \times 10^{-5} \mu$ is applied. The dimensionless drag coefficient is chosen as $\tilde{B}_{m}=0.0297$. The other simulation details are grouped in Table. 3 .

Instead of measuring dislocation velocity, another way to look closely at the different dynamic characteristics exhibited by the three $m$ cases is to measure an instantaneous dislocation wave speed' $\tilde{v}$ defined in the following; this is the nondimensional analog of $v$ in Sec. 4. This measure is useful especially when the applied load and the dislocation motions 
in $x$ are small. For different $m, \tilde{v}(x, t)$ takes the following form,

$$
\tilde{v}(x, t):=\left\{\begin{array}{l}
-\frac{\phi_{x}}{\tilde{B}_{m}}\left[\tau(x, t)-\tau^{b}(x, t)+\epsilon \phi_{x x}(x, t)\right], m=0 \\
-\frac{\operatorname{sgn}\left(\phi_{x}\right)}{\tilde{B}_{m}}\left[\tau(x, t)-\tau^{b}(x, t)+\epsilon \phi_{x x}(x, t)\right], m=1 \\
-\frac{1}{\tilde{B}_{m}} \frac{1}{\phi_{x}}\left[\tau(x, t)-\tau^{b}(x, t)+\epsilon \phi_{x x}(x, t)\right] \text { where } \phi_{x} \neq 0, m=2 .
\end{array}\right.
$$

$\tilde{v}_{a v}(t)$ is calculated by summing $v$ over the dislocation core at the specified time and dividing it by the core width. For our purposes here, the core width is chosen as $25 b$ which is much wider than the core defined in Fig. 5. We adopt this measure since the difference in dislocation profiles corresponding to the various $m$ dynamics is primarily at the very bottom, as already observed. We suspect that the $\tilde{v}(x)$ distribution at the bottom of the dislocation profile plays an essential role in determining dislocation motion (especially near the stepped 'core boundaries' when such steps exist), and a width of $25 b$ allows us to capture those features in sufficient detail in the cases being considered here.

The motivation for the definition of $\tilde{v}(x, t)$ is as follows: Suppose there exists a sufficiently smooth function $w$ that satisfies $w_{t}(x, t)=-w_{x}(x, t) s(x, t) . \quad s(x, t)$ may depend on its arguments through the values of the function $w$ and its derivatives but since the latter are known, it is appropriate to think of $s$ as a function directly of $(x, t)$. Then, using an elementary argument of the method of characteristics, along a curve $\hat{x}(t)$ in $(x, t)$ space defined by $\frac{d \hat{x}}{d t}=s(\hat{x}, t)$ starting from a point $\hat{x}\left(t^{*}\right)=x_{0}$, the value of $w$ is transported unchanged, i.e. $w(\hat{x}(t), t)=w\left(x_{0}, t^{*}\right)$. Thus at any instant of time, the transport velocity $s(x, t)$ may be interpreted as the instantaneous, local wave velocity at $(x, t)$; a positive sign of $s(x, t)$ implies transport in the $+x$ direction and a negative value implies transport in the $-x$ direction. Admittedly, for $m=2$ the definition of $\tilde{v}(x, t)$ in (49) is somewhat artificial, but this is not central to the point we make subsequently.

We note that the fields $\phi$ and $v$ in (10) satisfy the conditions outlined for $w$ and $s$, most likely including the smoothness assumption (for sufficiently large $\epsilon$ ).

We set up a simulation with initial condition being the unloaded NGB dislocation equilibrium of Fig. 5 with a constant-in-time applied load of $5 \times 10^{-5} \mu$. The dislocation stays motionless. We plot $\phi_{x}(x)$ and $\tilde{v}(x)$ along the layer in Fig.18. The $\tilde{v}(x)$ profile obtained here is definitely unfavorable to dislocation motion. The sign of $\tilde{v}(x)$ on each side of the centre of the core indicates that the left side of the dislocation core tends to move to the right while the right side to the left, and the core bottom tends to spread out. The simulation lasts for 0.4 s. This period is conservative since the NGL and NLS dynamics show motion within the first $0.05 \mathrm{~ns}$ under the same applied load. It is shown in Fig. 18(a) that the dislocation stays motionless during this period. The $\tilde{v}(x)$ profiles of Fig. 18 persist through out the simulation. Due to the small time-period of simulation in this dynamic test, we further examine the loaded NGB equilibrium as initial condition for the fast system. The applied load is still $5 \times 10^{-5} \mu$. The fast system is evolved for $0.42 \mathrm{~ns}$ and the dislocation under load shows no motion.

So far, based on the premise that 
- if the quasi-static system displays a loaded dislocation equilibrium arrived at from an unloaded dislocation equilibrium state and

- the dynamic equations also validate that the unloaded dislocation equilibrium does not begin to move under the action of the load on the fast time-scale,

then the system displays a Peierls stress, we have arrived at the following conclusions.

1. The NGB dynamics displays a Peierls stress effect for at least one initial condition (the unloaded NGB dislocation equilibrium). Interestingly, a curious 'stochastic' outcome arises out of our deterministic theory: two unloaded dislocation equilibria of very similar type at a gross level of reckoning (i.e. NLS and NGB dislocation equilibria) respond differently to loading and therefore predictions of Peierls stress for the NGB model may very well appear stochastic over repeated trials.

2. We have not been able to find a Peierls stress effect under load of the order of $\sim$ $10^{-5} \mu$ for any of the NGL, NLS, and NGB dynamics starting from the unloaded NGL dislocation equilibrium. We recall here that the results of Section 6.1 imply that the NGL and NLS quasi-static evolutions do not admit a loaded dislocation equilibrium (of the magnitude being discussed here) from their corresponding no-load dislocation equilibrium profiles as initial conditions.

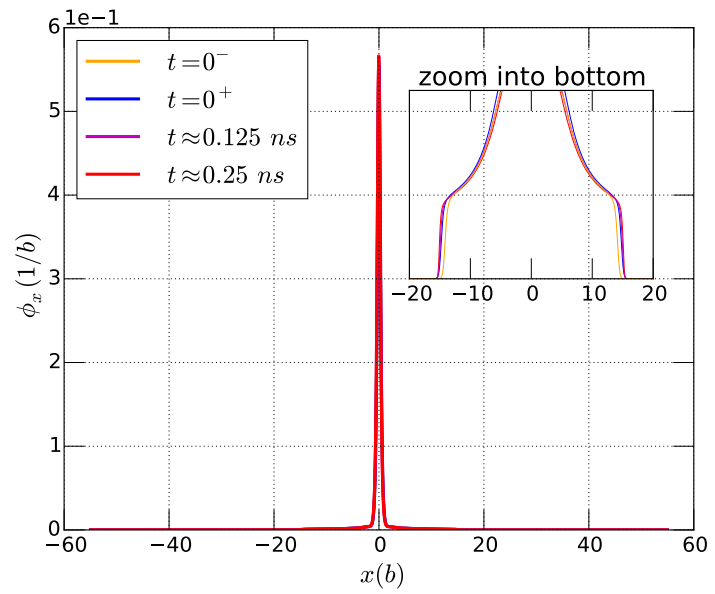

(a) $\phi_{x}$

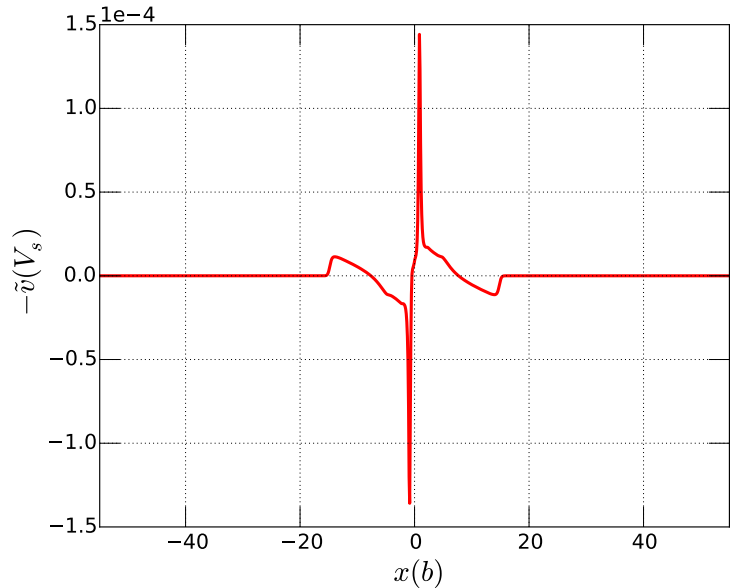

(b) $\tilde{v}(x)$

Figure 18: $\phi_{x}$ and $\tilde{v}(x)$ of $N G B$ dynamics. $t=0^{-}$stands for unloaded $N G B$ equilibrium which is used as initial condition. The instant of applying shear load $5 \times 10^{-5} \mu$ is indicated by $t=0^{+}$. The dislocation shows no motion but slight shape change at bottom. $\tilde{v}$ profile persists during the simulation.

In the following, we explore the dynamic behaviours of the NGL, NLS, and NGB systems under load $\tau^{a}=5 \times 10^{-5} \mu$, from a common initial condition of an unloaded NGL dislocation equilibrium shown in Fig. 5. The system runs on the fast time scale $\tilde{t}=b / V_{s} t$ and the simulation runs for around 1 nanosecond. 
It is observed that the NGB dislocation stays motionless during this period, while NGL and NLS move immediately after the load is applied.

Fig. 19(a) shows the initial, intermediate and final profiles of $\phi_{x}$ of the evolving NGB dynamics. $\tilde{v}(x)$ is shown in Fig. 19(b).

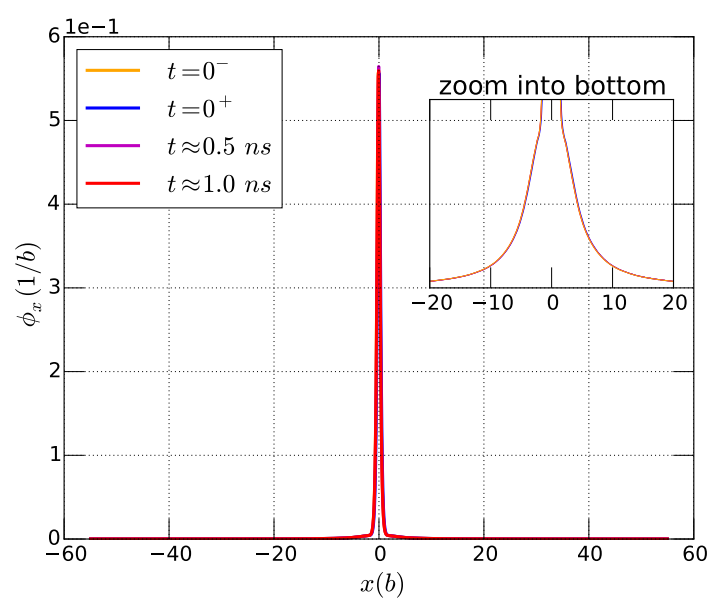

(a) $\phi_{x}$

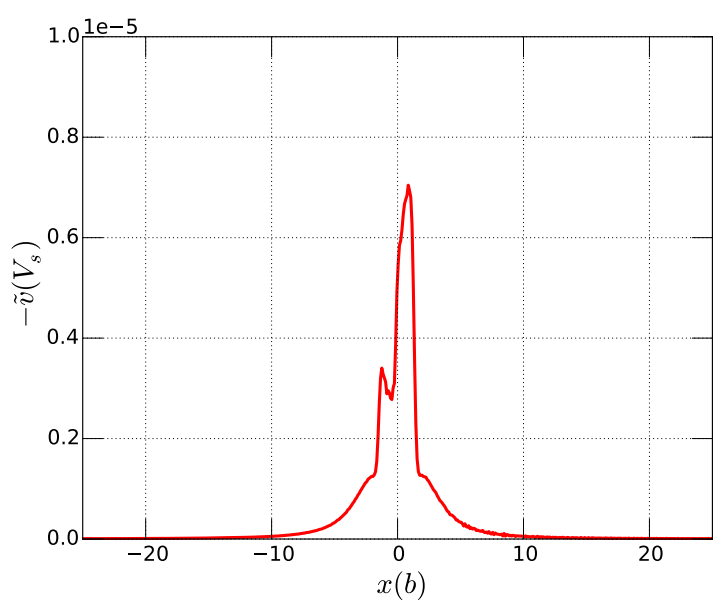

(b) $\tilde{v}(x)$

Figure 19: Fast system under load $5 \times 10^{-5} \mu$. Motionless dislocation (left) and field plot of $\tilde{v}(x)$ (right), starting from the NGL dislocation equilibrium indicated by $t=0^{-}$. The instant of applying shear load $5 \times 10^{-5} \mu$ is indicated by $t=0^{+}$.

Fig. 20 shows the comparison of $\tilde{v}_{a v}(t)$ between $m=0$ and $m=2$. NGL has a significantly larger wave speed than NGB, explaining why NGB does not move while NGL moves immediately after loading.

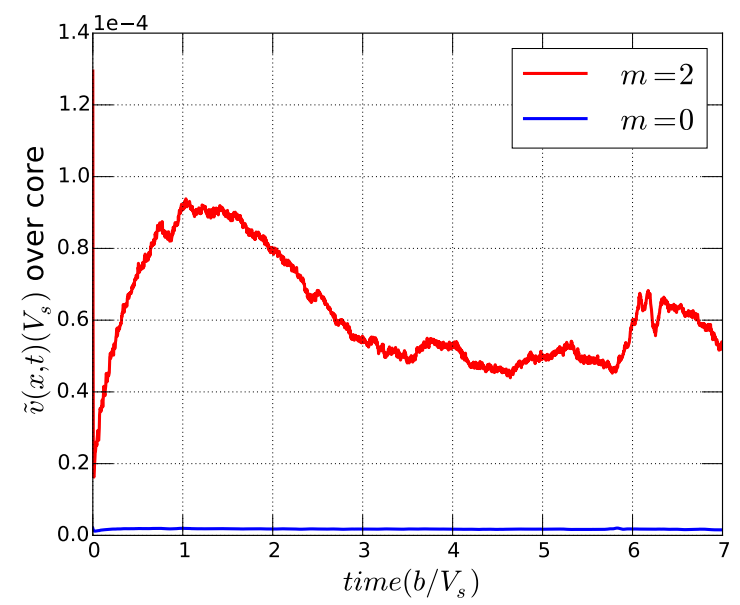

Figure 20: $\tilde{v}_{a v}$ under load $\tau^{a}=5 \times 10^{-5} \mu$, starting from $N G L$ dislocation equilibrium.

At each time step, the field shape of $\tilde{v}(x)$ also shows interesting differences between each $m$ case, as shown in Fig. $21(\tilde{v}(x)$ of NGB is shown in Fig. 19(b)). For NGL, the profile over 
only the dislocation core area is plotted). It is observed that $\tilde{v}$ at the dislocation boundaries of $m=2$ and $m=1$ are significantly larger than that of $m=0$. Within the core, the peak of NGB is approximately 8 times less than the other two. This difference in $\tilde{v}(x)$ contributes to the observed fact that NGB moves much slower than the NGL and NLS dislocations.

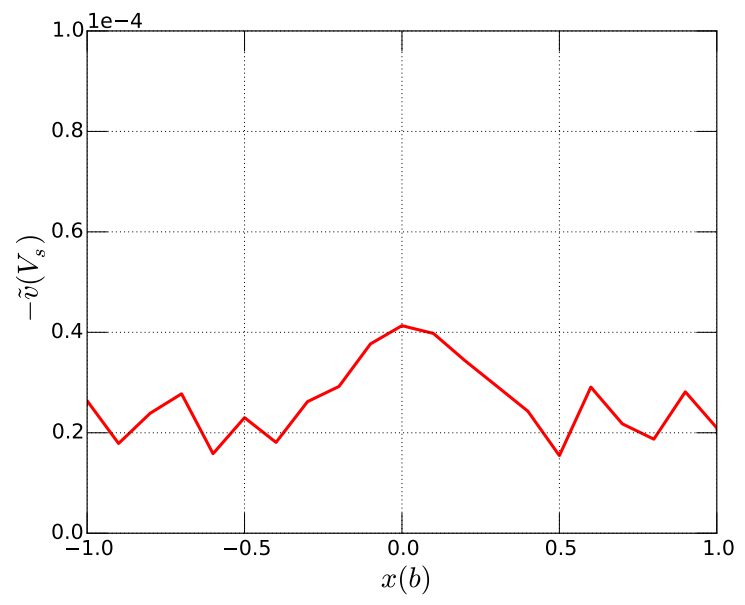

(a) $m=2$

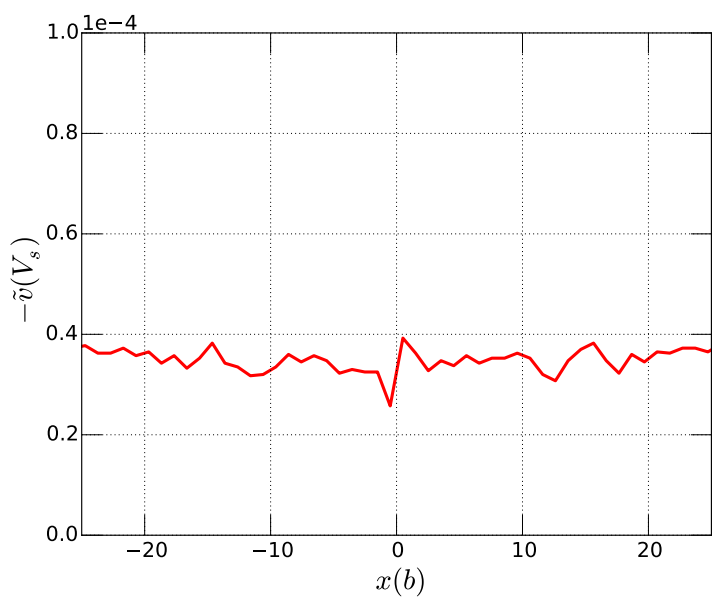

(b) $m=1$

Figure 21: Field plot of $\tilde{v}(x)$ of $N G L$ and $N L S$ under load $5 \times 10^{-5} \mu$, starting from NGL dislocation equilibrium.

It is instructive to compare the $\tilde{v}$ profiles presented in Figures 18 and 21 for $m=0$, under the load of $5 \times 10^{-5} \mu$. Even though the dislocation stays motionless in the latter case for the small time in which the observation has been done, the non-negative shape of the $\tilde{v}(x)$ curve coupled with the inability to find a loaded dislocation equilibrium in quasi-static equilibrium forces the conclusion that this situation reflects very slow motion of the type we discuss in Section 7.1.4.

Fig. 22 provides a gross sense of the dislocation velocity versus applied load curves for this regime of small loads. The results are gross because the exact governing equations in this case should be (16); we use those equations, but with the assumption that for the small magnitude of loads and vanishing loading rates involved, material inertia is unimportant. From this real time dynamics with $\tilde{B}_{m}=1$, the NGB dislocation does not show motion for $\tau^{a}<6.5 \times 10^{-5} \mu$. (Recall that a loaded dislocation equilibrium was also shown to exist for the quasi-static system (22)). The average velocity for the load of $2.5 \times 10^{-4} \mu$ is $5.99 \times 10^{-5} V_{s}$. For these results, the time scale and the velocity are physically meaningful. The other simulation details are kept the same as in Table 3. 


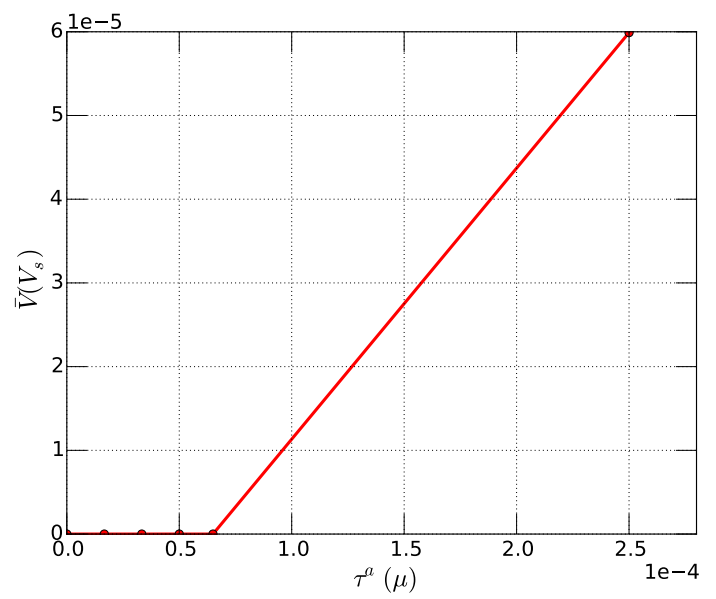

Figure 22: Dislocation velocity with respect to applied load at the threshold regime.

\subsubsection{Preliminary parametric study of factors affecting the Peierls stress}

- Effect of barrier height: We solve system (22) (with impulsively applied load), but now with some lower barrier heights. That is, the function $\eta$ in (13) is multiplied by a factor $f \in(0,1)$, and we consider the specific values

$$
\left\{\frac{2}{3}, \frac{1}{2}, \frac{1}{4}\right\} \text {. }
$$

The simulation details are kept the same as in Sec. 6. The tolerances specified by Eq. (38) remain in place. We find that $f=\frac{2}{3}$ is able to sustain both unloaded and loaded equilibria. The Peierls stress is found to be around $\sim 5 \times 10^{-5}$. (recall that $f=1$ gives a Peierls stress $6.5 \times 10^{-5} \mu$; this load magnitude leads to non-convergence of $|\triangle u|_{\infty}$ for $f=\frac{2}{3}$ ).

For $f=\frac{1}{2}$ and $f=\frac{1}{4}$, neither loaded nor unloaded equilibria can be attained.

- Effect of shape of $\eta$ profile: With reference to Fig. 23, a function of the type form $B$ (cf. [RBS92, Vit68, PDT10]), different from that specified in (13) represented as form $A$, is utilized. A Peierls stress of $7.5 \times 10^{-5} \mu$ is obtained for this case.

These results suggest, as expected, that the function $\eta$ plays an essential role in the prediction of the Peierls stress in models like ours. Interestingly, we find that for each of these variations, dislocation pre-equilibria for the NGB dynamics can always be attained. 


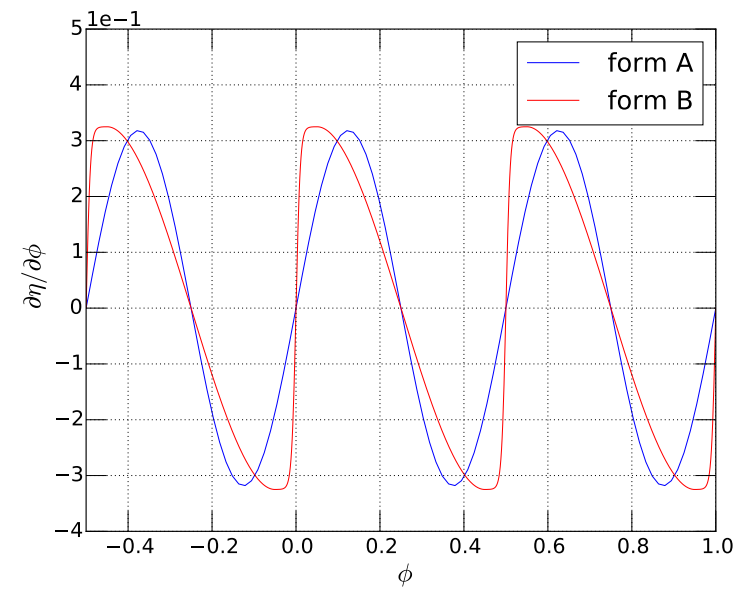

(a) $\frac{\partial \eta}{\partial \phi}$

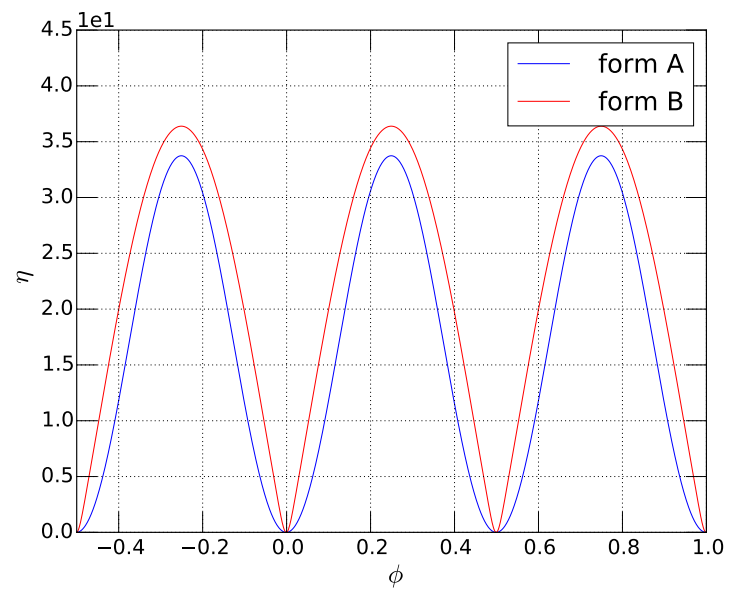

(b) $\eta$

Figure 23: Comparison of two types of $\eta$ functions and their derivatives.

Finally, although our formulation is based on a small deformation theory, the resultant plastic strain can be substantial due to the value of $\bar{\phi}=0.5$ corresponding to a well of the function $\eta$. In the following, we check whether an even larger plastic strain level, still specified by $\bar{\phi}$, can affect the Peierls stress results. We choose the value of $\bar{\phi}=1$, corresponding to a lattice invariant shear in a square lattice. The layer thickness is reduced to $1 b$ to maintain the initial Burgers vector to be $1 b$. The Peierls stress of this case is found to be $\sim 1 \times 10^{-5} \mu$. We again run a fast system (as described around Fig. 19) starting from unloaded NGB equilibrium for $t \approx 0.4 n s$ under the same applied shear load $1 \times 10^{-5} \mu$. The dislocation stays motionless during the simulation.

\subsubsection{Quasi-equilibrium aspects}

In Section 6.1, we have hinted that the quasi-static dynamics of our equations (19) can show sluggish behavior practically indiscernible from equilibria, when judged solely on the merit of the rate of evolution of some obtained states that we have termed pre-equilibria.

In this section, we discuss some features of such (unloaded) dislocation pre-equilibrium states and compare them with the states we have defined as unloaded dislocation equilibria (definitions in Section 6.1).

First, consider the dislocation pre-equilibria obtained from the common initial condition (37) (and utilized for the result in Figure 5). Specifically, we make the following observations:

1. Initially, the three quasi-static systems (19) (corresponding to $m=0,1,2$ ) evolve from the initial condition (37) with significant shape changes of $\phi_{x}$ and rates of change $\left|\phi_{s}\right|_{\infty}$. The profiles of $\phi_{x}$ then settle on the dislocation pre-equilibria configurations with the corresponding $\left|\phi_{s}\right|_{\infty}$ decreasing to $5 \times 10^{-5}$.

2. The displacement fields $\boldsymbol{u}$ corresponding to these dislocation pre-equilibria appear to have no discernible differences from their corresponding dislocation equilibrium displacement profiles. However, the difference in stress fields are on the scale of $10^{-4} \mu$. 
For demonstration, we show some comparisons of dislocation equilibria and pre-equilibria.

Fig. 24 and 25 compare the dislocation pre-equilibrium profiles for NGL and NLS with their dislocation equilibria. A first observation is that the NGL dislocation pre-equilibrium has almost the same shape as the NGL dislocation equilibrium. However, the difference for the NLS case can be observed at the bottom of Figure 25.

The profiles of the NGB dislocation at the iterations when $|F|_{\infty}=4.5 \times 10^{-5}$ and $|F|_{\infty}=$ $10^{-12}$ are both shown in Fig. 26. The comparison shows that the equilibrium dislocation shape is markedly different from the NGB dislocation pre-equilibrium with $|F|_{\infty}=5 \times 10^{-5}$, but the shape change is trivial from $|F|_{\infty}=4.5 \times 10^{-5}$ to $|F|_{\infty}=10^{-12}$. Specifically, the NGB dislocation pre-equilibrium has significantly rounded steps on both sides, while for the unloaded NGB dislocation equilibrium, one needs to zoom in to the bottom to see them (as shown in Fig. 8). Our results seem to suggest that it is possible that the NGB dislocation equilibrium may not be dynamically accessible from the NGB pre-equilibrium on any physically realistic time scale.

Based on the observations above, we define the NGB and NLS dislocation pre-equilibria as quasi-equilibrium states characterized by the fact that rates of evolution are extremely slow out of them and, in all likelihood, attainment of equilibrium from them, ceteris paribus, may not be possible on any physically attainable time-span. We emphasize that while we have discussed both quasi-equilibria and equilibrium states (under no load), it is not our intention to suggest that the demonstrated equilibria are the intended targets of attainment under evolution as $t \rightarrow \infty$ from their corresponding pre/quasi-equilibria. On the other hand, we have demonstrated that our dynamics (under no load), especially NGB, contain trajectories that have a rapid evolution regime, a sluggish regime indistinguishable from equilibria in a practical sense, and true equilibria.

As an example which involves more than one dislocation, we solve the pile-up problem of Fig. 14. A slight shift from the pre-equilibrium to the final solution is observed as shown in Fig. 27. The final equilibria seem to be closer to the exact solution. However, the shift is of magnitude no greater than $0.2 \mathrm{~b}$.

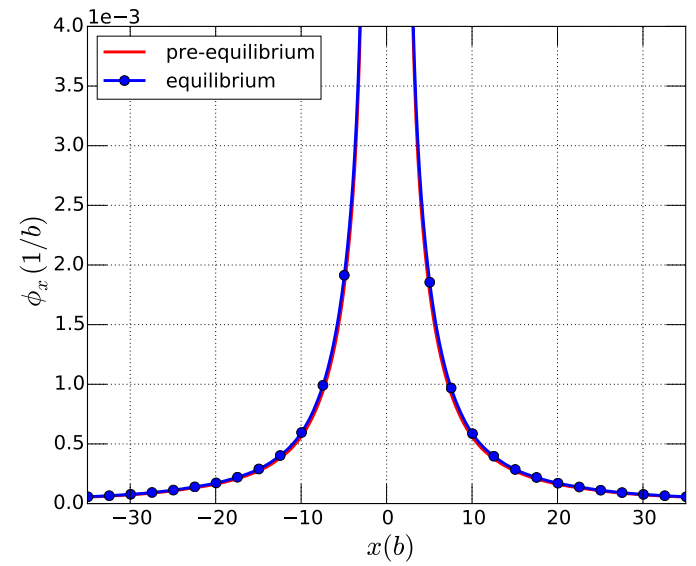

Figure 24: $\quad N G L$ equilibrium compared to NGL pre-equilibrium, zoom into the bottom.

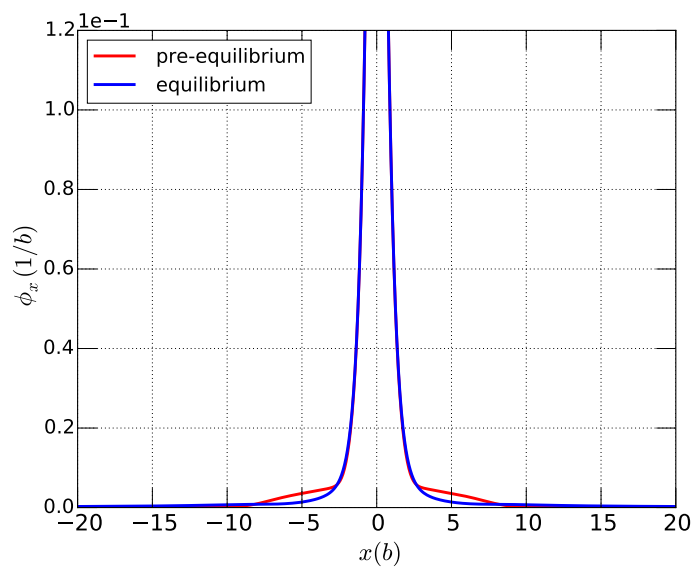

Figure 25: $N L S$ equilibrium compared to $N L S$ pre-equilibrium. 


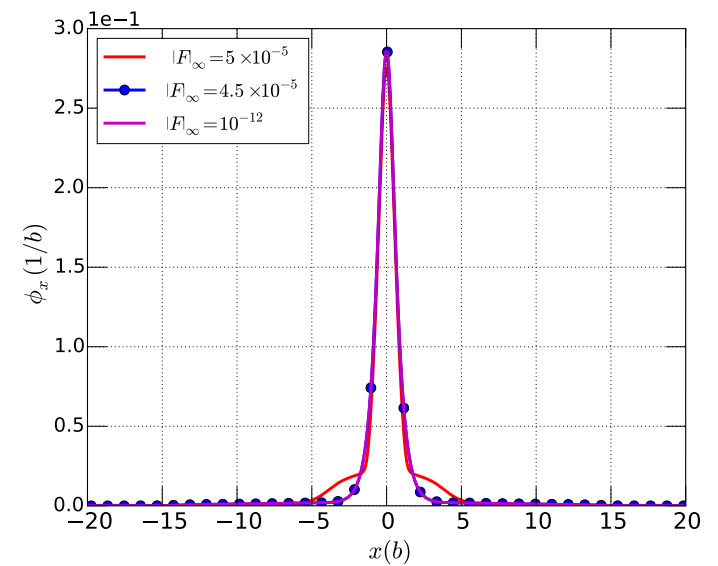

Figure 26: Comparison of $N G B$ preequilibrium $\left(5 \times 10^{-5}\right)$ and two moments during Newton iterations. NGB equilibrium is resolved to $|F|_{\infty}=10^{-12}$.

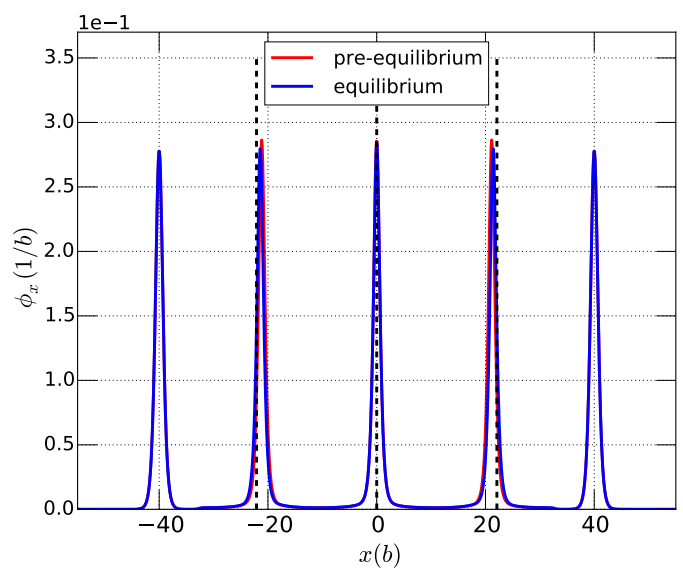

Figure 27: Pre-equilibrium compared to equilibrium of unloaded pile-ups. The black dotted lines indicate the analytical solutions.

\subsubsection{Peierls stress effects for $\tilde{B}_{m} \gg 1$}

We now consider dislocation motion in the presence of large drag. The question is whether one can still observe the Peierls stress effect on a slow time scale characterized by the large drag, under a slow loading rate.

The governing equations for $\tilde{B}_{m} \gg 1$ are (19). The system runs on the slow time scale determined by the non-dimensional drag number $\tilde{B}_{m}$. Here, the time scale and velocities are all physically meaningful. We implement a series of simulations with applied stress starting from $\tau^{a}=5 \times 10^{-5}$. The increments of applied load are kept very small especially when the loads are within $5 \times 10^{-3} \mu$.

The simulation set-up is kept the same as in Section 7.1.2, with parameters listed in Table 1 . We consider all three NGB $(m=0)$, NLS $(m=1)$ and NGL $(m=2)$ cases, with particular interest in the NGB $(m=0)$ case, since it is derived from the simplest kinetic assumption, and also because we have shown the Peierls stress effect for this dynamics in section 7.1.2 where $\tilde{B}_{m}$ is small.

The unloaded, dislocation pre-equilibrium profiles of Fig. 24, 25 and 26 are used as initial conditions for each $m$ dynamics. Uniform-in-time shear traction boundary conditions, as described in Eq. (40) are imposed. The average velocity $\bar{V}$ is measured by (48); $\bar{X}$ is conservatively chosen as $0.1 \mathrm{~b}$. The dislocation motion is thus quantified by measuring $\bar{V}$ and $\left|\phi_{s}\right|_{\infty}$. In addition, we define a 'slowness,' $S:=\bar{V}^{-1}$, to help visualize the Peierls stress effect.

The results are shown in Fig. 28(a) where $\bar{V}$ is plotted for applied loads smaller than $0.001 \mu$. The corresponding slowness data $S$ is shown in Fig. 28(b). A direct observation is that the dislocation has much slower motion with increasing $m$. The slowing down caused by $m$ is clearly demonstrated by fitting $S$ to a power law. We have fit $S$ vs. applied stress for the whole range of stresses below $5 \times 10^{-3} \mu$ based on the data of $\tau^{a}>5 \times 10^{-5}$, since the dislocation for NGB under load $5 \times 10^{-5}$ does not show any motions during the simulation. 
The plots of Fig. 28(a) and Fig. 28(b) display only the parts of that curve at the lower end of the spectrum - the fit is uniformly good in the entire range of stresses. The NGB $(m=0)$ dynamics has an exponent of 1.24 , much larger than that for NLS $(m=1)$ and NGL $(m=2)$. Since we are not able to perform infinitely-long time simulations, the conclusion is that the NGB dynamics starting from different NGL and NGB equilibrated profiles all show no or extremely slow motions.

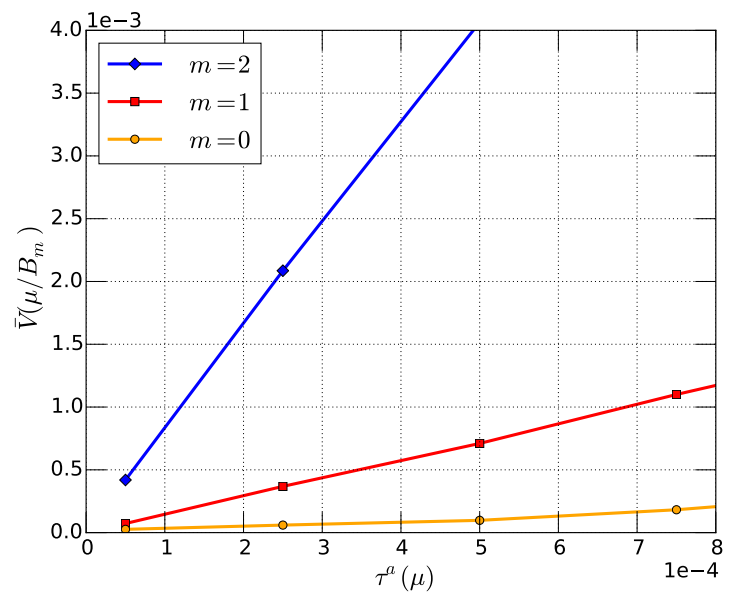

(a) zoom in view

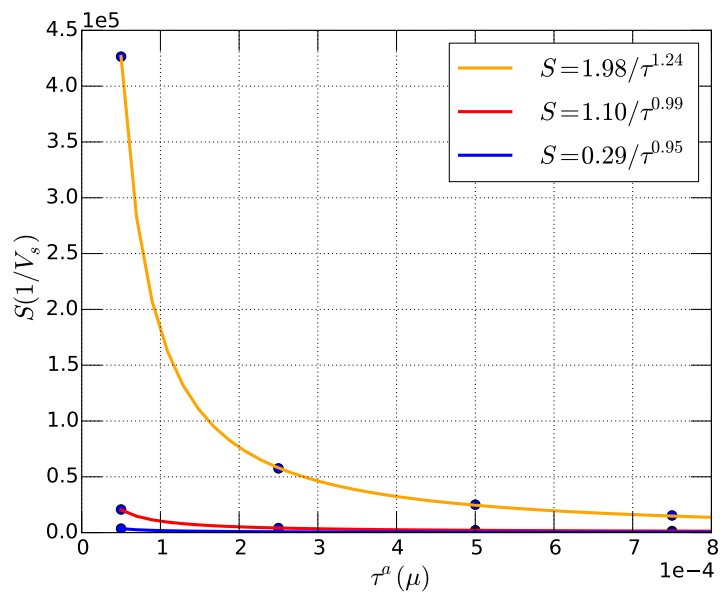

(b) slowness

Figure 28: Velocity and slowness recorded from simulations.

The existence of a Peierls stress in this $\tilde{B}_{m} \gg 1$ regime would be justified if $S \rightarrow \infty$ for a non-zero value of applied stress. We are unable to access smaller loads than shown because of the prohibitive time taken by these very long-time simulations. However, based on the power law fits of the available data, it can be quantitatively concluded that for the NGB dynamics both $\bar{V}(0)=0$ and $\frac{d \bar{V}}{d \tau^{a}}(0)=0$. For the NLS $(m=1)$ dynamics, the derivative may be considered a constant (making allowances for fitting), whereas for the NGL $(m=2)$ dynamics, $\frac{d \bar{V}}{d \tau^{a}}(0) \rightarrow \infty$. In addition, we show the plots of $\tilde{v}(x)$ and $\tilde{v}_{a v}(t)$ for the NGB simulation of this section under load $5 \times 10^{-5} \mu$. We conclude that in all probability there exists a Peierls stress effect in the NGB dynamics for $\tilde{B}_{m} \gg 1$ based on the following observations:

1. Fig. 29(a) shows that the NGB dynamics has a $\tilde{v}$ profile not favourable to dislocation motions, for the same reason as discussed around Fig. 18.

2. There is an obvious decreasing trend in $\tilde{v}_{a v}$ (Fig. 29(b)). The value of $\tilde{v}_{a v}$ at $s=3000$ is around $1 / 20$ of that at $s=0$. This suggests that the evolution of $\phi$ (specifically in $x$ direction) is slowing down.

3. Recall that we chose $0.1 b$ for $\bar{X}$ in determining the average velocity (48) - this is in part dictated by the extended times for which these simulations have to be run. This is a conservative choice and it is reasonable to expect the possibility of $S \rightarrow \infty$ for 
larger $\bar{X} \leq b$, especially because $\tilde{B}_{m} \gg 1$ corresponds to larger drag and hence smaller velocities.

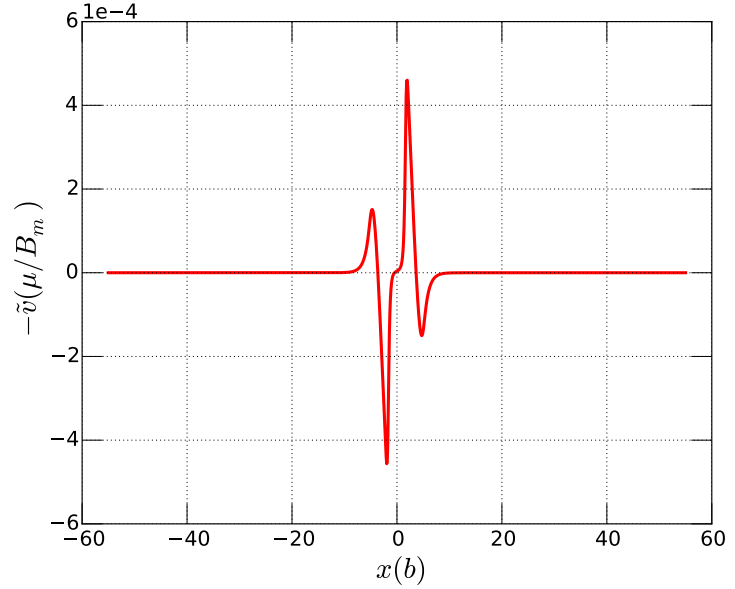

(a) $\tilde{v}(x)$.

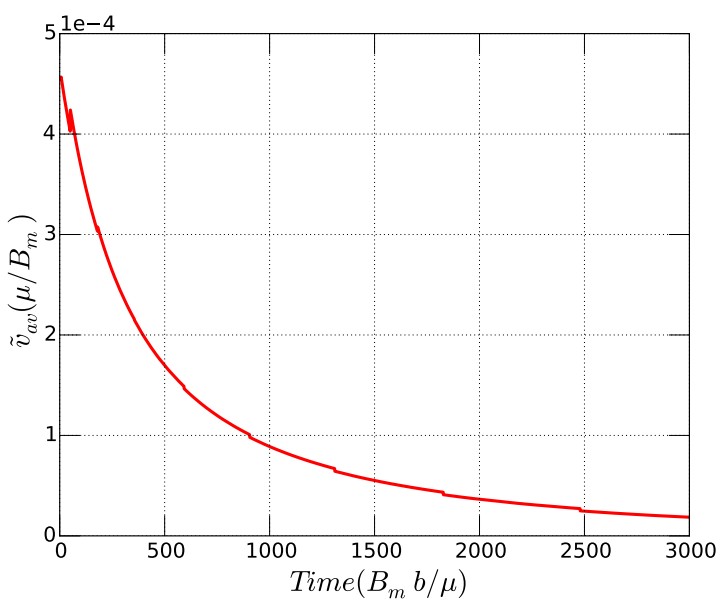

(b) $\tilde{v}_{a v}$.

Figure 29: $N G B$ dynamics of $\tau^{a}=5 \times 10^{-5} \mu$, $\tilde{v}$ field profile and $\tilde{v}_{a v}$ with respect to time.

\subsubsection{The Nabarro dipole}

As an instructive demonstration of the Peierls stress effect in the NGB $(m=0)$ dynamics, we model a configuration of two identical single dislocations with opposite signs (a dislocation dipole). We use the dynamics (19). According to Nabarro [Nab47], there exists a critical separation for a dipole to be in a stable equilibrium in a lattice, i.e., dipoles of closer separations annihilate by attractive forces, leaving the body dislocation-free. The essential mechanism of this phenomenon is the Peierls stress opposing the annihilating motion of the dipole. The simulation details are described in Table. 1, except that here we choose the diffusion strength $\epsilon=0.25$. The initial condition for $\phi$ is assumed to be

$$
\phi(x, 0)=\frac{\bar{\phi}}{2}(\tanh (x+r / 2)+\tanh (r / 2-x))
$$

$r$ being the dipole separation. Fig. 30 and Fig. 31 represent the equilibration with $r=50 b$ and $r=20 b$. Here we should mention the method we use to obtain the initial dipole of separation 20b: We solve two single dislocation traction free problems, one with a positive dislocation at $x=10$ and the other negative at $x=-10$. The two dislocations equilibrate separately. The superposition of the two equilibrium $\phi$ are then used as input to the $r=20 b$ simulation. It is observed that the dipole separated by $20 \mathrm{~b}$ cannot stay in equilibrium, with the dislocations of opposite sign attracting and annihilating each other. The dipole separated by $50 \mathrm{~b}$ gets equilibrated, i.e., although the dislocations are still subject to attractive forces from each other, the force is not large enough to draw the dipole closer, beating the instrinsic

sluggishness of the NGB dislocations. Fig. 32 shows how $\left|\phi_{s}\right|_{\infty}$ varies with respect to time for the equilibrated dipole. It is clear that the system evolves from the initial condition into 
an equilibrium state, for all practical purposes at least. The equilibrated dipole results in a static stress field as shown in Fig. 33.
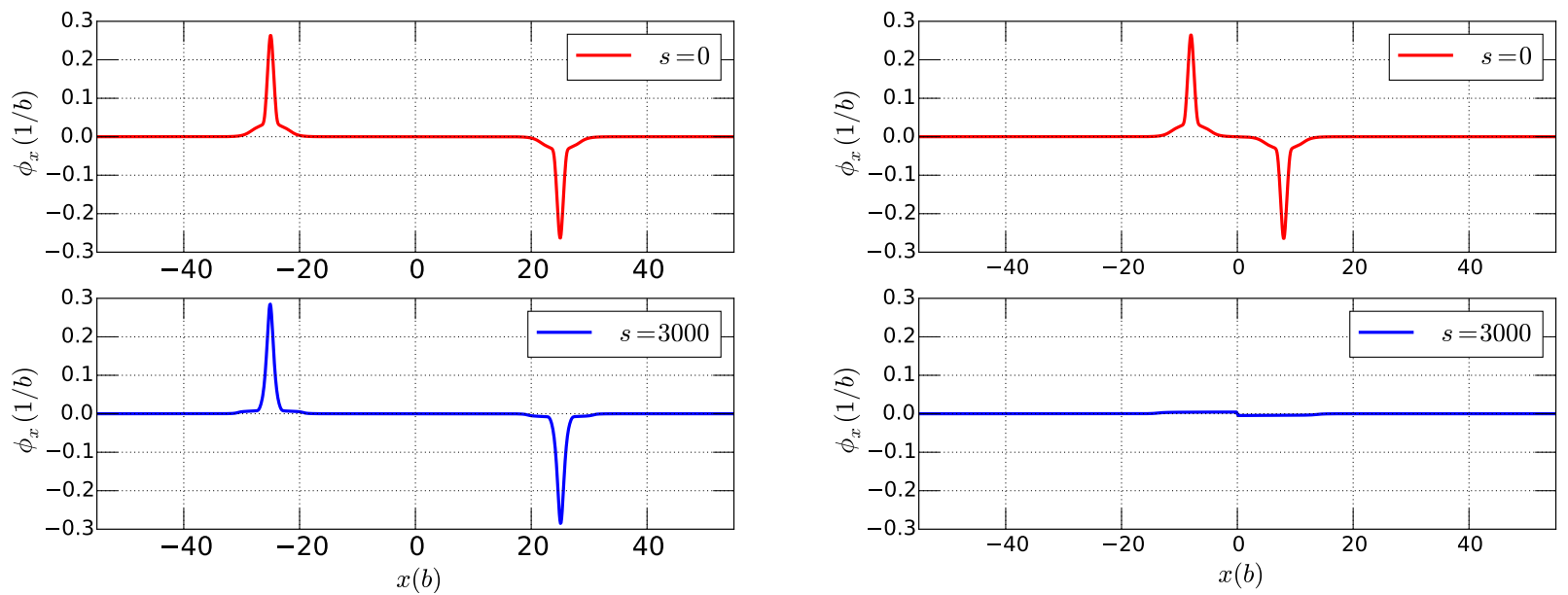

Figure 30: Equilibrated separated dipole.

Figure 31: Annihlated dislocation dipole.

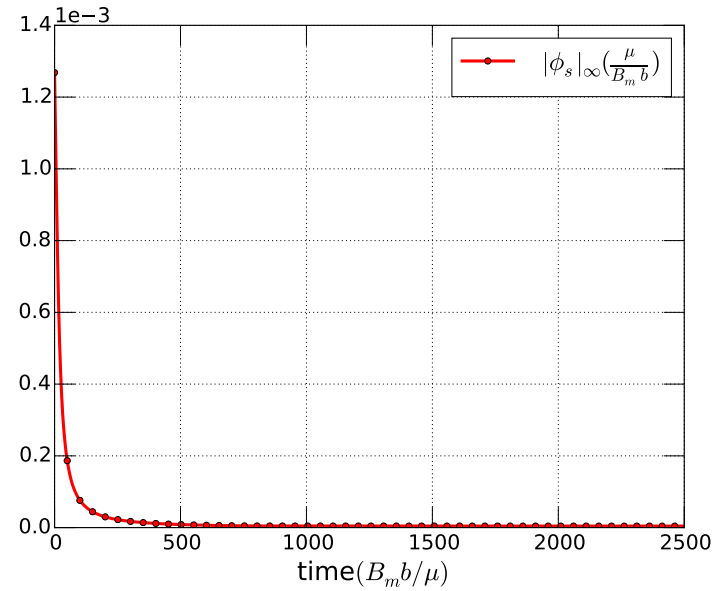

Figure 32: $\left|\phi_{s}\right|_{\infty}$ of equilibriated dipole.

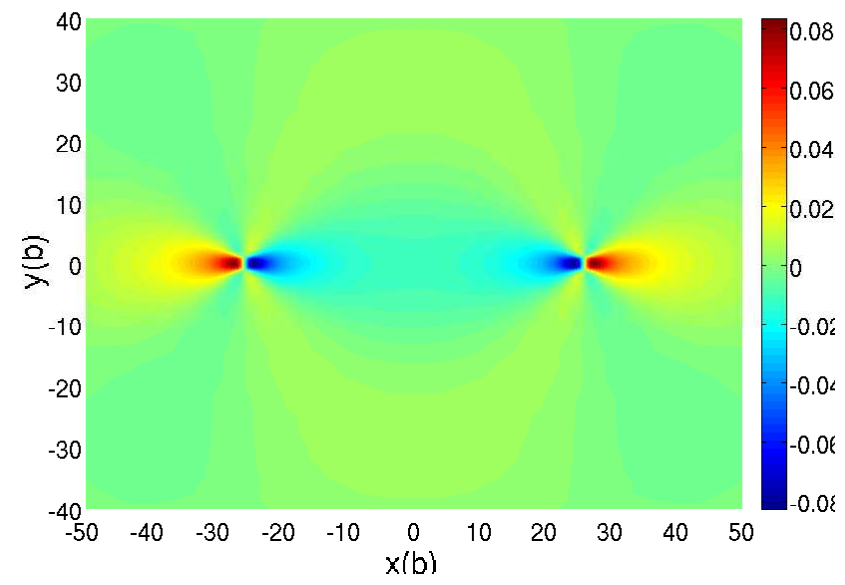

Figure 33: $\sigma_{12}$ of equilibrated dipole.

Applying a large enough shear load counterbalances the attractive Peach-Koehler forces and expands the dipole. Fig. 34 shows a series of snapshots of that scenario. 


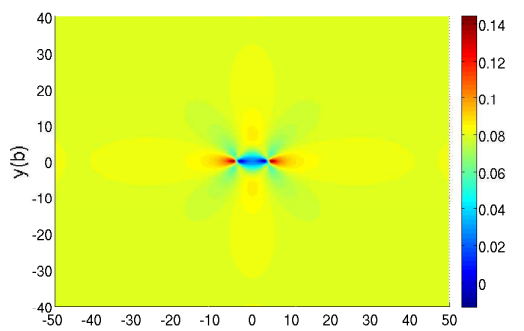

(a)

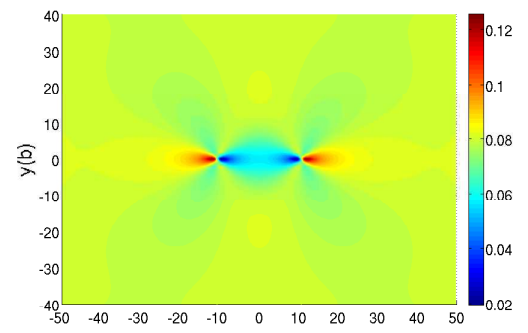

(b)

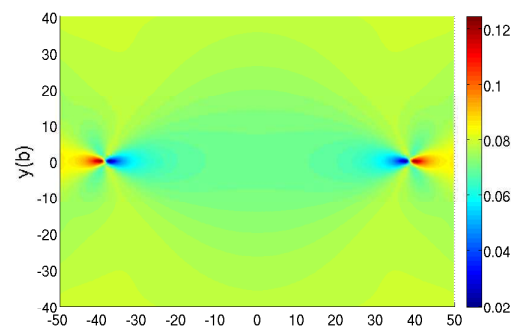

(c)

Figure 34: Dipole expands under shear stress.

\subsubsection{Idealized dislocation dissociation ${ }^{5}$}

Energy considerations dictate that it is possible for a dislocation to dissociate (split) into a pair, with the Burgers vectors of the dislocations in the pair adding up to that of the original dislocation. The argument from the classical theory essentially amounts to the following: Consider a large enough body containing, in the first instance, a single straight dislocation of Burgers vector magnitude $b$ and in the second instance two dislocations of the same sign as in the first case separated by a distance $R$, but each of Burgers vector magnitude $\frac{1}{2} b$. We now wish to compare the total energies of the two configurations; in the linear theory this is a question of comparing infinite magnitudes (even for finite bodies). Nevertheless, with the usual hand-waving of the linear theory related to accounting for the core energy, if in the first case the energy is written as $a b^{2}$ then in the second case the energy is $2 a\left(\frac{1}{2} b\right)^{2}+c \frac{b^{2}}{R}$, where $a, c$ are positive constants. For $R$ sufficiently large, the energy of the second configuration is smaller than the first; therefore, dissociation is favored. Such a phenomenon is actually observed in closed-packed crystals with low stacking fault energy. We show in this section that, without any additional constitutive rules, our model is able to simulate an idealized dislocation dissociation process.

We use the NGB dynamics and keep the simulation details as for calculations reported in Fig. 5 with the following modifications:

$$
\begin{aligned}
& \phi(x, t=0)=\bar{\phi} \tanh (a x) \\
& \epsilon=0.025, a=\sqrt{\mu / 4 \epsilon},
\end{aligned}
$$

which represents an initial dislocation with a Burgers vector magnitude of $2 b$. Fig. 35 shows the process of it splitting into two separated dislocations, each of Burgers vector magnitude $b$. The corresponding re-distribution of plastic strain caused by the dislocation dissociation is also shown. In this way, it locally minimizes the total system energy. The initial dislocation profile spans three successive wells of $\eta$ (to achieve its Burgers vector magnitude), and we observe from the simulation that each dislocation in the dissociated pair spans two wells of $\eta$ as they move apart. The separated single dislocations have repulsive forces in between, and push each other away after dissociation.

\footnotetext{
${ }^{5}$ We thank Mr. Chiqun Zhang for his help with the simulations in this Section.
} 


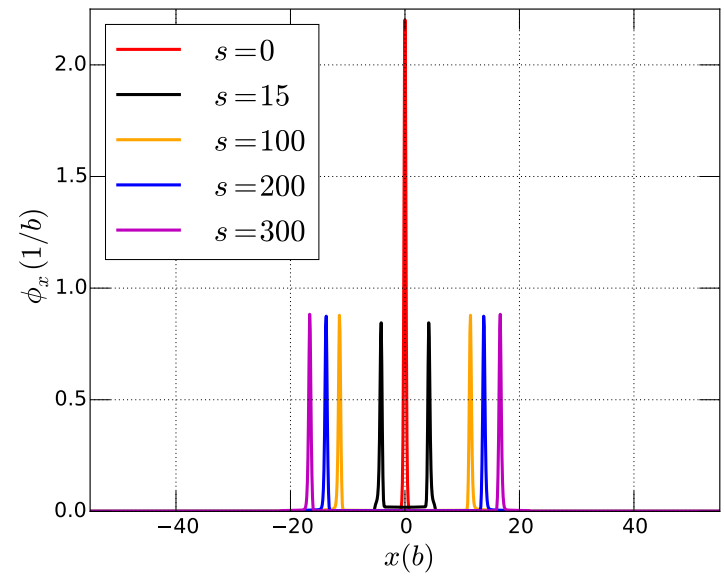

(a) $\phi_{x}$

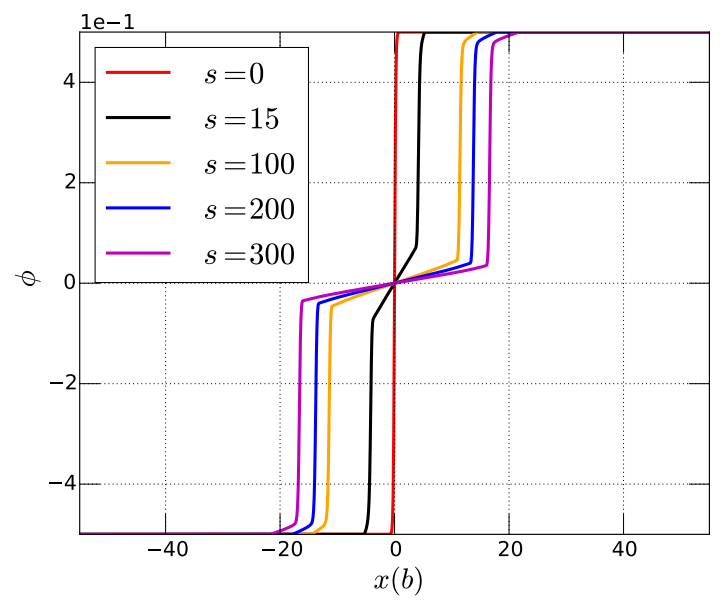

(b) $\phi$

Figure 35: An example of modeling dislocation dissociation.

\subsection{Loading and unloading of a single dislocation}

Here we show the response of the model to a loading-unloading cycle. We continue to use the slow dynamics (19). We apply a simple shear traction boundary condition (40) to move the dislocation from its equilibrated position. The load is then instantaneously removed. The NGB dynamics shows interesting/peculiar behavior as presented in Fig. 36(a) at three critical moments during the simulation: the top, where $s=0$, the dislocation is equilibrated. The middle panel shows the dislocation driven to its furthest location, and at that moment the load suddenly drops to zero. Without any exterior stress, the dislocation spike creeps backwards with diminishing speed to finally equilibrate at the position shown in the bottom panel. The equilibrium shape is similar to the shape at the start with some differences; we have not let the equilibration process run long enough to conclude whether the height of the peak returns to the original value or not. As can be seen, the equilibrium shape of the dislocation is significantly different from that during motion. The history of $\left|\phi_{s}\right|_{\infty}$ on the right also reflects this process: the sudden increase of $\left|\phi_{s}\right|_{\infty}$ is caused by the sudden load drop. After unloading, $\left|\phi_{s}\right|_{\infty}$ decreases sharply, which suggests the re-(pre)equilibration process of the load-free dislocation. In contrast, the unloading behaviour of the NLS $(m=1)$ and the NGL $(m=2)$ dislocations are different. There, the dislocations continue to move towards the left boundary after the load is taken away, presumably due to the attraction of the free boundary. While the recoil of the NGB dislocation gives us some pause in the context of expected behaviour based on classical ideas, its equilibration is another example of the Peierls stress effect in the NGB case. We have checked that if an NGB dislocation under no load is placed closer to the boundary, it does move towards the boundary. We have also checked that for all three NGL, NLS, and NGB dislocations, the core-averaged layer stresses are positive, indicating an attraction towards the left boundary. 


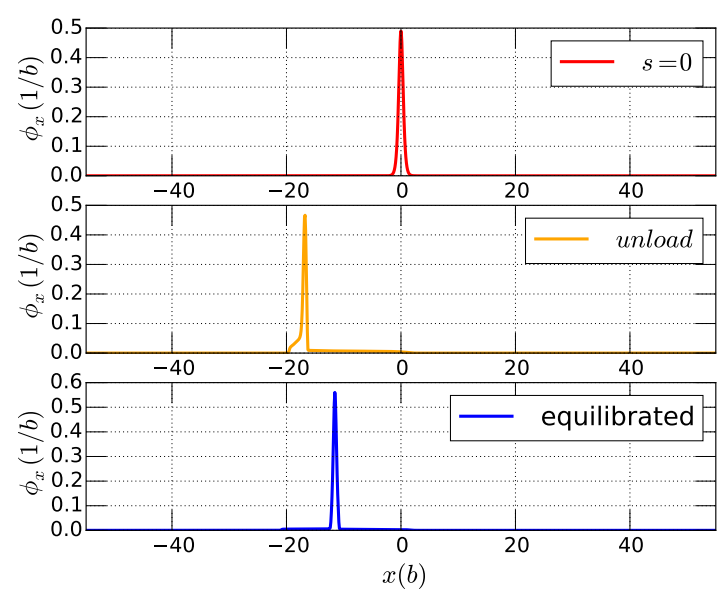

(a) Motion of a dislocation under loading-unloading. top: free traction equilibrium ready to move. middle: dislocation moves to the left most when load is dropped to zero. bottom: dislocation moves back and re-gains its new equilibrium state.

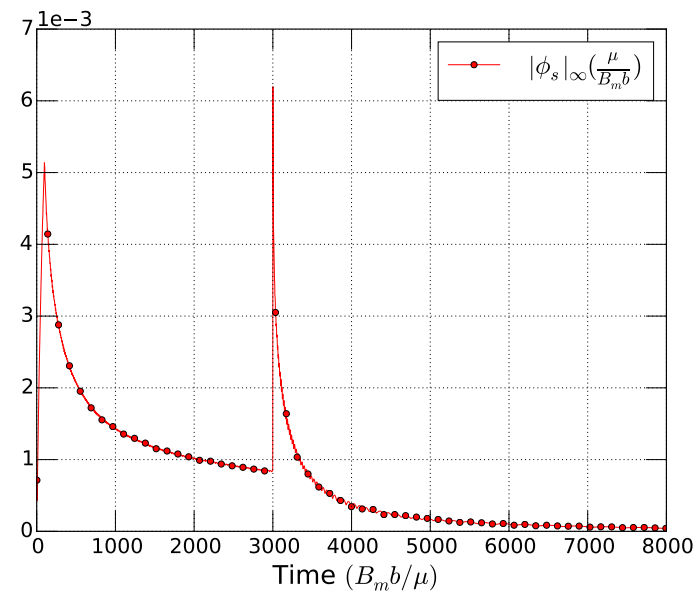

(b) $\left|\phi_{s}\right|_{\infty}$ corresponding to (a)

Figure 36: Loading then unloading $(m=0)$.

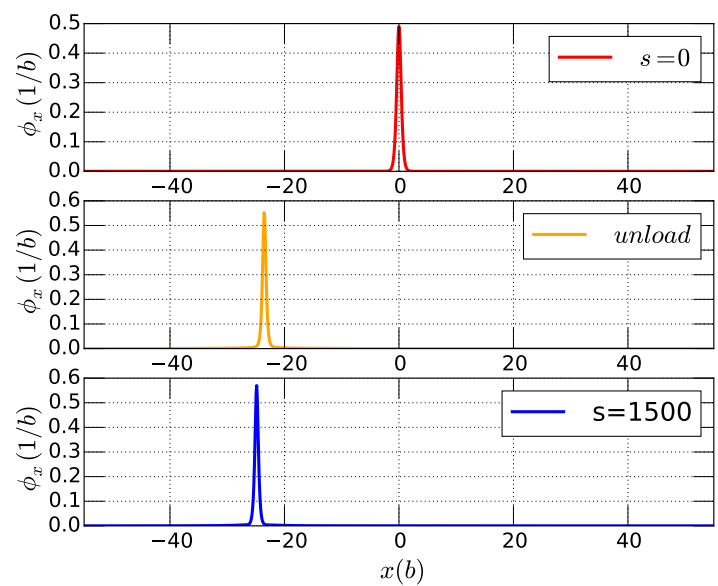

(a) loading then unloading of $m=1$.

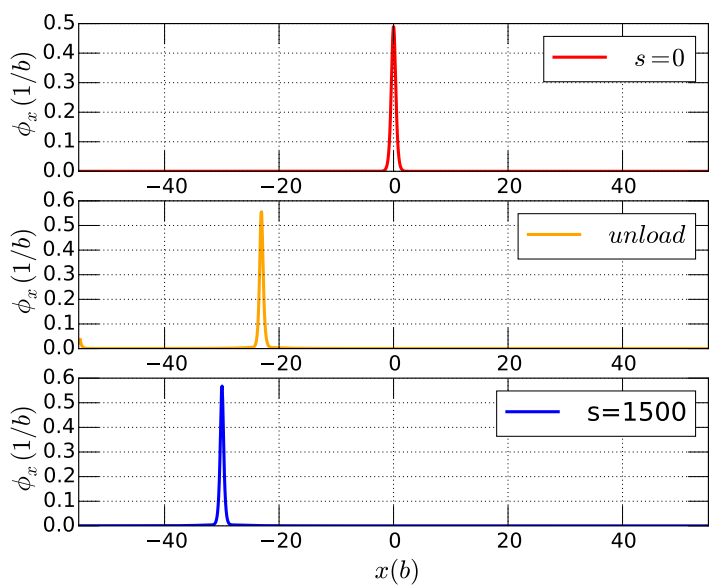

(b) loading then unloading of $m=2$.

Figure 37: Loading then unloading $(m=1,2)$. The dislocations moves to the left under simple shear traction same as $m=0$. However, as the load is removed, $m=1$ and $m=2$ both keep moving to the left boundary. Specifically, NGL moves faster than NLS as shown in the bottom snapshot at the same nondimensional time $s=5500$.

\subsection{Convergence Test}

The Peierls stress results are verified by convergence tests. First, we take an NLS $(m=1)$ system with applied load $\tau^{a}=0.005 \mu$ from Section 7.1 .5 and show convergence with respect 
to the mesh of $\left|\phi_{s}\right|_{\infty}$, the dislocation displacement, and the average speed $\bar{V}$. These three measurements together quantify the motion of a dislocation. All the simulation details can be found in Table. 1. The use of transition elements around the layer keeps the overall computation time from growing significantly as the layer gets refined. Let $h$ denote the width of layer elements and we create meshes with $h=0.2,0.1,0.05,0.025(b)$. We should also point out that the finite difference grid is refined accordingly in the meantime and the time steps are automatically refined according to Eq. (27). Although convergence of $\left|\phi_{s}\right|_{\infty}$ is a more stringent test than the other two, even in Fig. 38(a), one can observe the trend of convergence of $\left|\phi_{s}\right|_{\infty}$ with decreasing $h$. Fig. 38(b) shows the position of the dislocation peak $\bar{X}$ vs. $s$. Processing Fig. 38 (b) by dividing $\bar{X}$ with time gives the averaged speed $\bar{V}$. As shown in $38(\mathrm{c}), \bar{V}$ converges with $h$, but with a notable disturbance when $\bar{X}<0.5 b$ for all $h$.

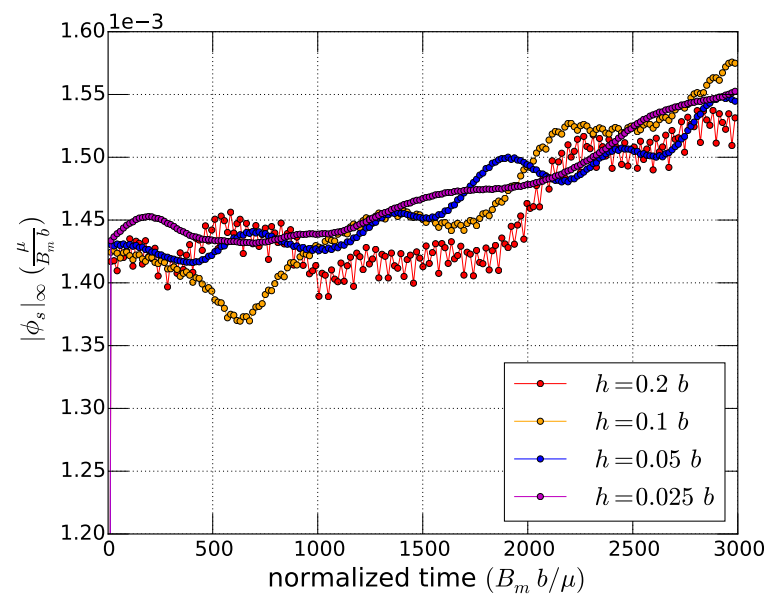

(a) $\left|\phi_{s}\right|_{\infty}$

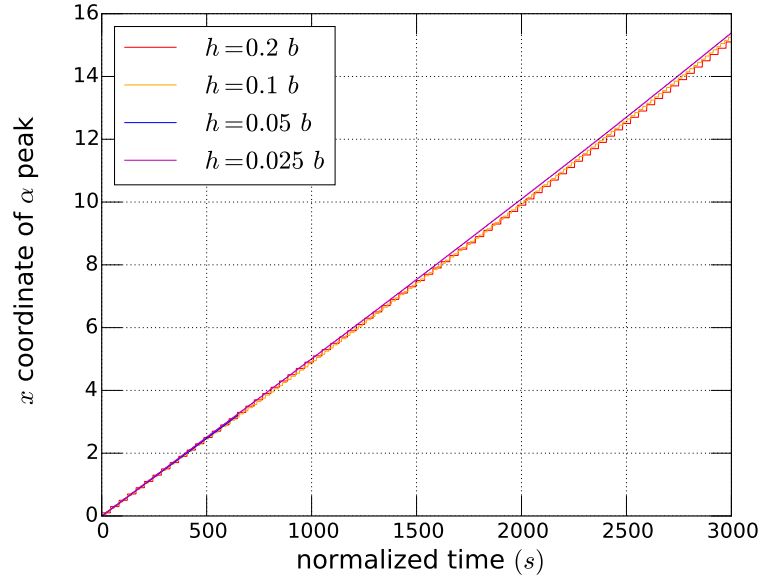

(b) dislocation position

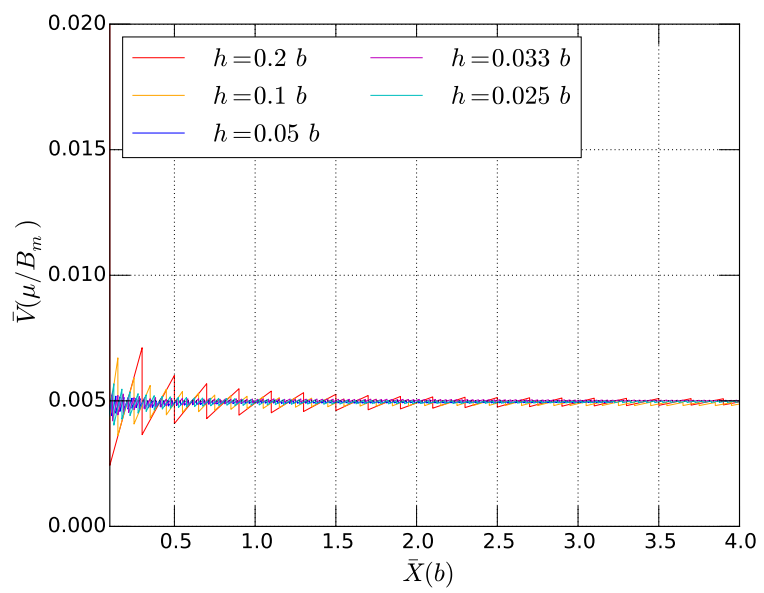

(c) $\bar{V}$

Figure 38: Convergence test.

For the equilibrium results obtained with the Quasi-Newton scheme, we run the same 
problem described around Fig. 9 on a well-refined mesh: $h=0.025 b$. We test applied load from $\tau^{a}=5 \times 10^{-5} \mu$ to $\tau^{a}=10^{-4} \mu$. The results are the same as obtained from $h=0.1 b$. The equilibrated $\phi_{x}$ with $\tau^{a}=6.5 \times 10^{-5} \mu$ for both $h=0.1 b$ and $h=0.025 b$ are plotted in Fig. 39. The two profiles are on top of each other.

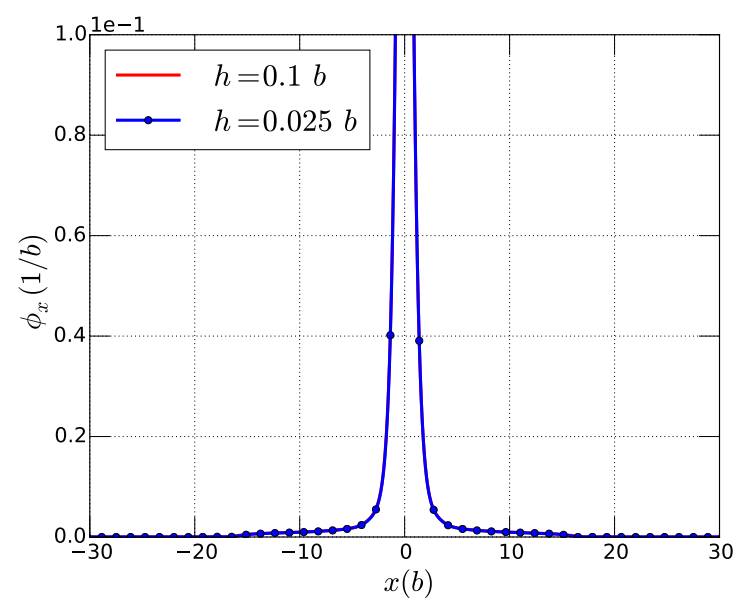

Figure 39: Dislocation profile resulted from well-refined mesh compared to $h=0.1 b$.

The following tests show the convergence of the Peierls stress results at lower applied load region. We have tested three NGB $(m=0)$ cases from section 7.1.5 where $\tau^{a}$ takes the values $5 \times 10^{-5} \mu, 2.5 \times 10^{-5} \mu$ and $5 \times 10^{-4} \mu$. The simulation lasts for $s=3000$. The dislocation does not show motion until $s=3000$ for all $h$ cases when $\tau^{a} \leq 2.5 \times 10^{-4} \mu$. With $\tau^{a}=5 \times 10^{-4}$, the velocities for all $h$ cases are close but vary with a difference up to $1.0 \times 10^{-5} \mu / B$ (between $h=0.2 b$ and $h=0.033 b$ ).

To complete our convergence analysis for Peierls stress results, we have also verified that the velocity is not affected by the domain size $(W$ and $H)$. This is done by running the system (19) with applied load of $5 \times 10^{-4} \mu$ on a domain twice as large (and we keep $h=0.1 b$ on this larger domain) and comparing results with that from the smaller domain; the results remain unchanged. Finally, we argue that the symmetric attraction forces from the left and right boundaries counterbalance each other at the center of the domain, and therefore the stress threshold (the onset of motion) is not affected by the vertical free boundaries.

\subsection{Annihilation of a double pile-up}

We solve a pile-up having opposite signs on each half of a slip plane, namely a double pileup. The following simulation is a demonstration of the evolution of a double pile-up of dislocations in a traction free body, using the NLS $(m=1)$ slow dynamics associated with (19). The simulation parameters of this section are grouped in Table. 4. 


\begin{tabular}{|l|l|}
\hline parameter name & value \\
\hline domain width $(W)$ & $220 b$ \\
domain height $(H)$ & $180 b$ \\
No. of elements & 33960 \\
No. of nodes & 34038 \\
core-energy strength $(\epsilon)$ & 0.25 \\
Young's modulus $(E)$ & $70 G P a$ \\
shear modulus $(\mu)$ & $26 G P a$ \\
Burgers vector $(b)$ & $4.05 \times 10^{-10} \mathrm{~m}$ \\
Shear wave speed $\left(V_{s}\right)$ & $V_{s}=3.13 \mathrm{~km} / \mathrm{s}$ \\
\hline
\end{tabular}

Table 4: Simulation details for double annihilation pile-up.

$n=14$ dislocation spikes are initialized at $s=0$, spaced by $5 b$, i.e., the $i^{\text {th }}$ dislocation has a peak at $\left\{x_{i}=(-35+5 i) b\right\}, 0 \leq i \leq n$.

As the system evolves, the inner dislocations are drawn closer immediately and annihilate under the combined effects of attractive forces (of dislocations of opposite signs) and repulsive force (of dislocations of the same sign). The motions of others are driven by a complex timedependent stress field. Fig. 40 shows the trajectories of each dislocation in this process. Note that the outside two trajectories spread outwards initially before bending and becoming parallel in the end due to the annihilation of all the other dislocations. The two outer dislocations are separated by $132 b$ at the moment that all other dislocations annihilate as shown in the scenario of Fig. 31. Their evolution towards each other becomes extremely slow due to the small magnitude of the attraction force between them due to the large distance of separation. Also, the boundary attractions are extremely small based on the analysis around Fig. 37(a).

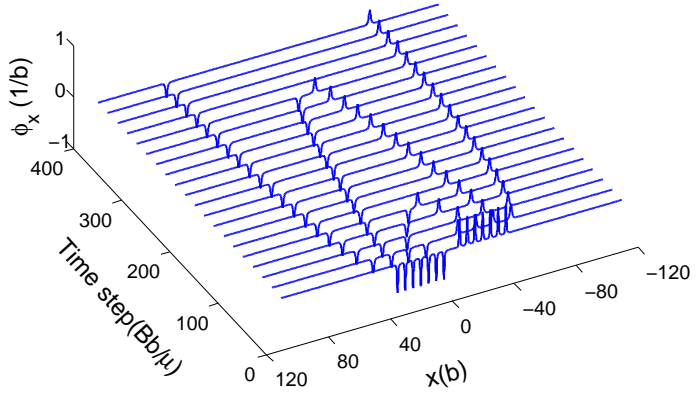

Figure 40: Double pile-up in traction free body.
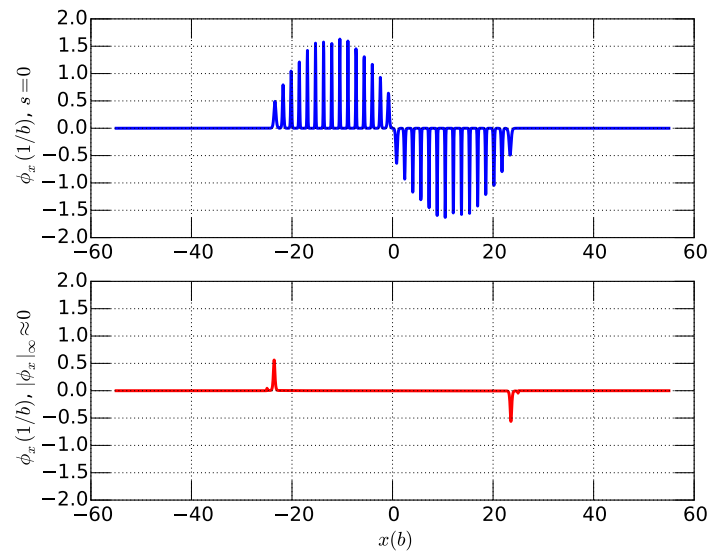

Figure 41: Almost completely continuous double pile-up with ends fixed (top). The middle dislocations annihilate (bottom).

By inserting more dislocations into the previous array, one could simulate almost continuously distributed pile-ups. The outer two dislocations on both sides are pinned. We use a 
$[110 b \times 90 b]$ domain for this case. The initial distribution of dislocations is shown in the top panel of Fig. 41. All the other simulation details are kept the same as in Table. 4. Fig. 41 bottom shows the final state of the double pile-ups. It shows that all dislocations annihilate except the pinned ones on the outside. This example also demonstrates that although $\boldsymbol{\alpha}$ is a conserved variable, the norm of $\boldsymbol{\alpha}$ is not. There exist several continuum dislocation plasticity models (motivated from discrete dislocation methodology) that insist on conservation of both $\boldsymbol{\alpha}$ and norm of $\boldsymbol{\alpha}$, while this is a straightforward counterexample.

\section{Dislocation motion in dynamic deformations}

In this section we examine the capability of our theory of modeling dislocation-related phenomena in the presence of material inertia. In all instances, the system (16) constitutes the governing equations. The theory has modeling relevance for phenomena at the atomic as well as geologic length scales. With computational capabilities of the type demonstrated

in this paper, our theory appears to be a relatively straightforward and robust tool to probe such questions in fair generality that otherwise require delicate analytical skills (cf. [Wil65, Mur63, AR02, Fre98, Mar11, Pel10, Pel11]).

\subsection{Subsonic, intersonic, and supersonic dislocation motion}

We investigate dislocation velocity versus applied load phenomena in a specific setting, with special interest in probing the sub-inter-supersonic regime of dislocation motion within our model. Because of the peculiar singularities at the speed of sound that occur in the relatively few complete solutions available in the linear elastic theory of dislocations, e.g. [AR02], questions of supersonic dislocation motion have generally been outside the realm of exploration for the classical theory of dislocations. Of course, if it is assumed that a stress wave in a purely linear elastic medium is the only carrier of signals that fail the material to form a dislocation core, then it is indeed physically unreasonable to expect the core to be able to travel faster than the linear elastic wave speeds of the material.

However, a system of numerical experiments performed by Gumbsch and Gao [GG99], motivated by transonic shear cracks observed in high-speed impacts [RSC99], reported the possibility of a transonic dislocation speeding up into the supersonic regime in a highly prestressed body. The MD experiment studied the motion of dislocations nucleated within a thin strip subjected to simple shear pre-strain. The dislocation velocity varies from subsonic to supersonic magnitudes according to the level of enforced strains.

A sketch of the MD velocity vs. applied shear strain obtained in Gumbsch and Gao's MD experiments is shown in Fig. 42. In the sketch, only the black dots are of concern since our problem is slightly different from theirs; e.g. the MD experiment involves the process of dislocation nucleation from a notch tip. The velocity data has two sudden transitions close to the shear wave and pressure wave velocity $V_{s}$ and $V_{p}$. The very nonlinear velocity vs. applied strain relationship is also noteworthy. The objective of this section is to qualitatively compare the results from our model with these MD results. To this end, we set up the initialboundary value problem in a manner similar in principle to the MD simulations in [GG99]. The model has a domain of size $110 b \times 80 b$, discretized uniformly. The simulation parameters 


\begin{tabular}{|l|l|}
\hline controlling parameter & value \\
\hline domain width $(W)$ & $110 b$ \\
domain height $(H)$ & $90 b$ \\
mesh refinement & $351 \times 91$ \\
core-energy strength $(\epsilon)$ & 1 \\
Young's modulus $(E)$ & $70 G P a$ \\
shear modulus $(\mu)$ & $26 G P a$ \\
Burgers vector $(b)$ & $4.05 \times 10^{-10} \mathrm{~m}$ \\
shear velocity $\left(V_{s}\right)$ & $3.13 \mathrm{~km} / \mathrm{s}$ \\
pressure velocity $\left(V_{p}\right)$ & $6.32 \mathrm{~km} / \mathrm{s}$ \\
\hline
\end{tabular}

Table 5: Simulation details.

are grouped into Table. 5.

The simulations are performed by the following steps:

1. First, we solve for the displacement field, $\boldsymbol{u}_{s}$, of a static dislocation-free body subjected to Dirichlet boundary conditions defined by (53):

$$
\overline{\boldsymbol{u}}=\Gamma\left(x_{2}+H\right) \boldsymbol{e}_{1}, \text { on } \partial \Omega
$$

This would result in a homogeneous simple shear strain of $0.5 \Gamma\left(\boldsymbol{e}_{1} \otimes \boldsymbol{e}_{2}+\boldsymbol{e}_{2} \otimes \boldsymbol{e}_{1}\right)$ in a linear elastic body. We define $\varepsilon_{12}=0.5 \Gamma$ and use it to denote different simple shear loading cases of time-independent Dirichlet boundary conditions in this Section.

2. Second, we solve for an equilibrated $\phi_{e}$ and displacement field $\boldsymbol{u}_{e}$ of a quasi-static, traction-free problem with a single dislocation at the point $(40 b, 0)$.

3. Dynamic simulations are performed according to (16) with initial conditions $\boldsymbol{u}(\boldsymbol{x}, 0)=$ $\boldsymbol{u}_{s}(\boldsymbol{x})+\boldsymbol{u}_{e}(\boldsymbol{x})$ and $\phi(\boldsymbol{x}, 0)=\phi_{e}(\boldsymbol{x})$. The initial condition provides a pre- $\varepsilon_{12}$-stressed body with an equilibrated dislocation ready to move. The Dirichlet boundary condition on the displacement fields in 1) above is maintained during the dynamic run - in the terminology of plasticity theory, we simulate a 'relaxation test' in the presence of significant inertia. The average dislocation velocity is recorded for each case of $\varepsilon_{12}$ (the average dislocation velocity is defined as in Eq. (48)). Since the load is applied through a Dirichlet boundary condition, no external power is supplied to the dislocation for it to maintain a constant velocity, i.e., the dislocation speed has to drop as it moves due to dissipation. However, in our numerical experiments, the decrease in speed turns out to be very slow. Specifically, we choose different $\bar{X}$ s in Eq. (48) and the variation in the resulting average dislocation velocity is found to be negligible.

Our hypothesis for the subsequent numerical experiments is the following: we work with a non-singular, dissipative model that, by design, satisfies the second law of thermodynamics (globally). Here, dislocation motion is the only dissipative mechanism. Thus, the larger the reservoir of elastic energy available from the pre-straining, the greater may be the propensity of the dislocation to move faster to dissipate the energy. This seems to suggest that significant velocities can be attained based on the level of pre-straining. 
Additionally, the following analysis ${ }^{6}$ shows the possibility of supersonic stress waves in an initially stressed body in the context of linearized elasticity. Let $\boldsymbol{P}, \boldsymbol{\tau}$ and $\stackrel{\circ}{\boldsymbol{\tau}}$ be the first Piola-Kirchhoff stress, the Kirchhoff stress and the contravariant convected rate of Kirchhoff stress, defined with respect to an initially stressed configuration (treated as the current configuration). Then, $\dot{\boldsymbol{P}}$ evaluated at the current configuration is given by

$$
\begin{aligned}
\dot{\boldsymbol{P}} & =\dot{\boldsymbol{\tau}} \boldsymbol{F}^{-T}-\boldsymbol{\tau} \boldsymbol{L}^{T} \boldsymbol{F}^{-T} \\
& =\dot{\boldsymbol{\tau}}-\boldsymbol{\tau} \boldsymbol{L}^{T} \\
& =\stackrel{\circ}{\boldsymbol{\tau}}+\boldsymbol{L} \boldsymbol{\tau}+\boldsymbol{\tau} \boldsymbol{L}^{T}-\boldsymbol{\tau} \boldsymbol{L}^{T} \\
& =\stackrel{\circ}{\boldsymbol{\tau}}+\boldsymbol{L} \boldsymbol{\tau} .
\end{aligned}
$$

Balance of linear momentum on the current configuration can be written as

$$
\operatorname{div}[\stackrel{\circ}{\boldsymbol{\tau}}+\boldsymbol{L} \boldsymbol{\tau}]=\rho \ddot{\boldsymbol{v}}
$$

and we assume the elastic constitutive relationship

$$
\stackrel{\circ}{\tau}=C: D
$$

where $\boldsymbol{C}$ is the $4^{\text {th }}$ order linear elasticity tensor and $\boldsymbol{D}=\frac{1}{2}\left(\boldsymbol{L}+\boldsymbol{L}^{T}\right)$. Let $M_{i j k l}=C_{i j k l}+$ $\tau_{l j} \delta_{i k}$. Assuming plane-wave solutions of the form $v_{i}=p_{i} e^{I\left(n_{r} x_{r}-c t\right)}$, where $\boldsymbol{p}$ is the velocity mode of the plane wave (polarization), $\boldsymbol{n}$ the direction of propagation of the velocity wave, and $I=\sqrt{-1}$, one has the characteristic equation

$$
\left[M_{i j k l} n_{l} n_{j}-\rho c^{2} \delta_{i k}\right] p_{k}=0
$$

for the $\operatorname{speed}(\mathrm{s}) c$ and polarization $\boldsymbol{p}$. Choosing now a homogeneous state of initial stress given by $\boldsymbol{\tau}=\tau \boldsymbol{a} \otimes \boldsymbol{b}$ with $\boldsymbol{a}$ and $\boldsymbol{b}$ as arbitrary unit vectors, one finds that longitudinal waves $(\boldsymbol{p}=\boldsymbol{n})$ propagate with speed

$$
c_{L}=\sqrt{\frac{\lambda+2 \mu+\tau(a \cdot n)(b \cdot n)}{\rho}}
$$

and transverse waves $(\boldsymbol{p}$ in plane normal to $\boldsymbol{n})$ propagate with speed

$$
c_{T}=\sqrt{\frac{\mu+\tau(a \cdot n)(b \cdot n)}{\rho} .}
$$

Note that the linear elasticity analogs of $c_{L}$ and $c_{T}$ are $V_{p}=\sqrt{\frac{\lambda+2 \mu}{\rho}}$ and $V_{s}=\sqrt{\frac{\mu}{\rho}}$. Thus $c_{L}>V_{p}>V_{s}$ as well as $c_{T}>V_{p}>V_{s}$ are definite possibilities for suitable states of initial stress, even without resorting to full-blown nonlinear theory.

\footnotetext{
${ }^{6}$ We acknowledge discussions with Profs. C. S. Man and R. W. Ogden on the possibility of elastic waves in linearized elasticity supersonic with respect to linear elasticity.
} 


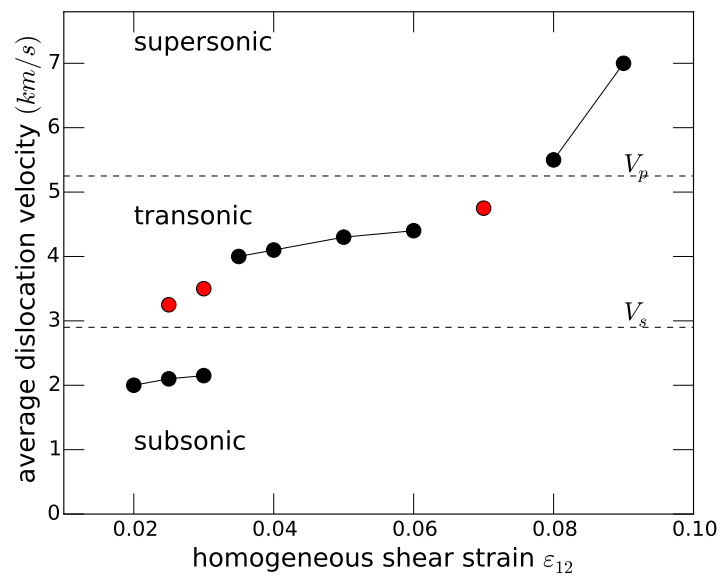

Figure 42: Sketch of the MD results from Gumbsch and Gao [GG99], reprinted with permission from AAAS. Dashed lines indicate relevant acoustic wave speeds.

\subsubsection{Linear Elasticity}

The dynamic simulations discussed above are performed with the equations (16), utilizing the constitutive equation (12), which implies a linear elastic relationship between the stress and the elastic strain. Fig. 43 shows the relationship between the applied strain and the average dislocation velocity. Numerical experiments are done on a body of size $[110 b \times 90 b]$ which is uniformly meshed and refined so that stress waves propagating through the entire body can be accurately captured. The simulation details are grouped in Table 5.

$m=0, m=1$, and $m=2$ cases are considered. Unlike the quasi-static simulations, here we need a physical value for the dimensionless drag coefficient. For $m=1$ and 2, we adopt the value $\tilde{B}_{m}=0.0297$ (see the discussion surrounding (20)). For $m=0$, we choose $\tilde{B}_{m}=0.0037$, based on fitting to eliminate a disparity in magnitude of results in Fig. 45, to be discussed subsequently (note that it is reasonable to expect $\tilde{B}_{m}$ to depend on $m$ ).

Figure 43 shows that while defeating the linear elastic wave speeds is possible for the dislocation velocity in our model based on the level of pre-strain, the overall velocity-stress relationship is not qualitatively close to the MD results of [GG99]. In particular, the plateaus around $V_{s}$ and $V_{p}$ have not been captured. The shear stress wave profile propagating through the body in the case of $\varepsilon_{12}=0.125$ is shown in Fig. 44. A Mach cone is observed behind the moving dislocation (from right to left) with an angle of approximately $38.7^{\circ}$. 


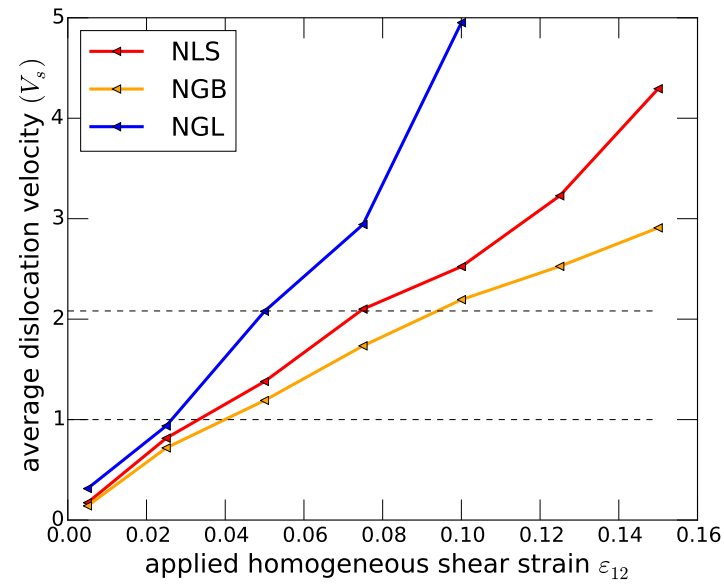

Figure 43: Dislocation velocity with homogeneous shear strain $\varepsilon_{12}$ applied to geometry linear body.

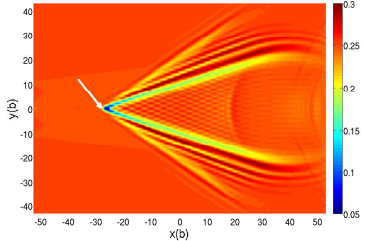

(a)

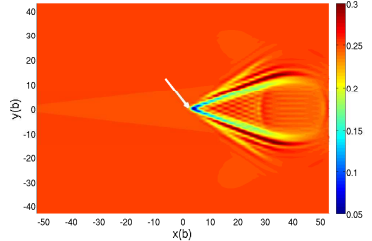

(b)

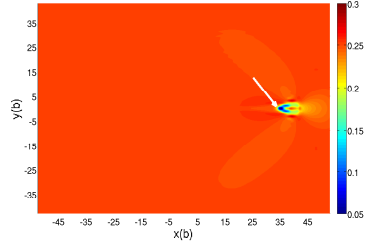

(c)

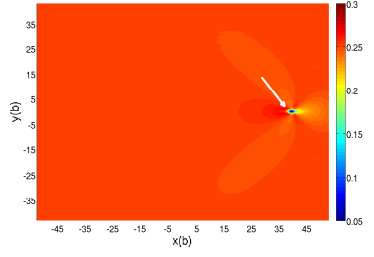

(d)

Figure 44: From right to left, shear stress wave around a moving dislocation under applied shear strain $\varepsilon_{12}=0.125$. The arrows denote the positions of the dislocation core.

\subsubsection{Nonlinear Elasticity and Finite Deformation}

In order to understand at least some effects of using nonlinear elasticity and finite deformation vis-a-vis the 'prediction' of the [GG99] MD results on high-speed dislocation motion, we adopt a grossly simplified version of the full finite deformation theory of Field Dislocation Mechanics [Ach04, Ach07, Ach11]. Roughly speaking, we allow for geometric nonlinearities in the total deformation and elastic constitutive equation, but ignore the kinematic nonlinearities in the evolution of the dislocation density; while this suffices for our purpose here, such nonlinearities are important, e.g. in the prediction of dislocation nucleation [GAM15].

We adopt the simplest St. Venant Kirchhoff model of nonlinear elasticity. Let $\boldsymbol{T}$ be Cauchy stress. The 1st Piola-Kirchhoff stress is defined through the elastic distortion $\boldsymbol{F}^{e}$ and the deformation gradient $\boldsymbol{F}$ as

$$
\boldsymbol{P}=J \boldsymbol{T} \boldsymbol{F}^{-T}=J \boldsymbol{F}^{e}\left(\boldsymbol{C}: \boldsymbol{E}^{e}\right) \boldsymbol{F}^{e T} \boldsymbol{F}^{-T}
$$

where $\boldsymbol{E}^{e}=0.5\left(\boldsymbol{F}^{e T} \boldsymbol{F}^{e}-\boldsymbol{I}\right)$. We solve for balance of linear momentum in the reference configuration (this allows us to use the computational set-up for solving for the displacement fields in the small deformation setting without significant change). Motivated by single slip 
kinematics of crystal plasticity theory, we assume the plastic distortion to take the form

$$
\boldsymbol{F}^{p}=\boldsymbol{I}+\boldsymbol{U}^{p}
$$

where $\boldsymbol{I}$ is the second order identity tensor and $\boldsymbol{U}^{p}$ is defined by (8). Also, we write $\boldsymbol{F}=$ $\boldsymbol{I}+\operatorname{Grad} \boldsymbol{u}$, with all spatial derivative operators with respect to the reference configuration. Next, we assume the multiplicative decomposition of $\boldsymbol{F}$ :

$$
\boldsymbol{F}=\boldsymbol{F}^{e} \boldsymbol{F}^{p}
$$

where $\boldsymbol{F}^{e}$ is the elastic distortion.

We choose the stored energy density function $\psi$ (per unit mass) to be

$$
\psi=\frac{1}{\rho_{0}}\left(\hat{\psi}\left(\boldsymbol{F}^{e}, \boldsymbol{\alpha}\right)+\eta\left(\boldsymbol{F}^{p}\right)\right)=\frac{1}{\rho_{0}}\left(\frac{\lambda}{2} \operatorname{tr}\left(\boldsymbol{E}^{e}\right)^{2}+\mu t r\left(\boldsymbol{E}^{e 2}\right)+\frac{1}{2} \epsilon \boldsymbol{\alpha}: \boldsymbol{\alpha}+\eta\left(\boldsymbol{F}^{p}\right)\right),
$$

where $\rho_{0}$ is density of the reference configuration. We have

$$
\begin{aligned}
& \boldsymbol{L}=\boldsymbol{L}^{e}+\boldsymbol{L}^{p} \\
& \boldsymbol{L}^{e}=\dot{\boldsymbol{F}}^{e} \boldsymbol{F}^{e-1}, \quad \boldsymbol{L}^{p}=\boldsymbol{F}^{e} \dot{\boldsymbol{F}}^{p} \boldsymbol{F}^{p-1} \boldsymbol{F}^{e-1},
\end{aligned}
$$

and $\boldsymbol{L}$ is the velocity gradient. Then the dissipation in this finite deformation case takes the form (cf Eq. (8)).

$$
\begin{aligned}
\mathcal{D} & =\int_{B} \boldsymbol{T}: \boldsymbol{L}-\frac{\rho}{\rho_{0}}(\dot{\overline{\hat{\psi}}}+\dot{\eta}) d v \\
& =\int_{B_{0}} J \boldsymbol{T}:\left(\boldsymbol{L}^{e}+\boldsymbol{F}^{e} \dot{\boldsymbol{F}}^{p} \boldsymbol{F}^{p-1} \boldsymbol{F}^{e-1}\right) d v_{0}-\int_{B_{0}}\left(\frac{\partial \psi}{\partial \boldsymbol{F}^{e}}: \dot{\boldsymbol{F}}^{e}+\frac{\partial \eta}{\partial \boldsymbol{F}^{p}}: \dot{\boldsymbol{F}}^{p}+\frac{\partial \psi}{\partial \boldsymbol{\alpha}}: \dot{\boldsymbol{\alpha}}\right) d v_{0} \\
& =\int_{B_{0}}\left(J \boldsymbol{T}-\frac{\partial \psi}{\partial \boldsymbol{F}^{e}} F^{e T}\right): \boldsymbol{L}^{e} d v_{0}+\int_{B_{0}} J \boldsymbol{T}:\left(\boldsymbol{F}^{e} \dot{\boldsymbol{F}}^{p} \boldsymbol{F}^{p-1} \boldsymbol{F}^{e-1}\right) d v_{0}-\int_{B_{0}} \frac{\partial \eta}{\partial \boldsymbol{F}^{p}}: \dot{\boldsymbol{F}}^{p}+\frac{\partial \psi}{\partial \boldsymbol{\alpha}}: \dot{\boldsymbol{\alpha}} d v_{0} \\
& =\int_{L_{0}}\left\{\left(J \boldsymbol{F}^{e T} \boldsymbol{T} \boldsymbol{F}^{e-T}\right): \dot{\boldsymbol{F}}^{p} \boldsymbol{F}^{p-1}-\frac{\partial \eta}{\partial \boldsymbol{F}^{p}}: \dot{\boldsymbol{F}}^{p}\right\} d v_{0}+\int_{L_{0}} \frac{\partial \psi}{\partial \boldsymbol{\alpha}}: \operatorname{curl}(\boldsymbol{\alpha} \times \boldsymbol{V}) d v_{0} \\
& =\int_{L_{0}}\left(J \boldsymbol{F}^{e T} \boldsymbol{T} \boldsymbol{F}^{-T}-\frac{\partial \eta}{\partial \boldsymbol{F}^{p}}\right): \dot{\boldsymbol{F}}^{p} d v_{0}+\int_{L_{0}} \frac{\partial \psi}{\partial \boldsymbol{\alpha}}: \operatorname{curl}(\boldsymbol{\alpha} \times \boldsymbol{V}) d v_{0},
\end{aligned}
$$

where the stress function $\boldsymbol{T}$ defined over the whole body is chosen as

$$
\boldsymbol{T}=\frac{1}{J} \frac{\partial \psi}{\partial \boldsymbol{F}^{e}} \boldsymbol{F}^{e T} .
$$

Linear momentum balance, expressed in the reference configuration, gives

$$
\operatorname{Div} \boldsymbol{P}=\operatorname{Div}\left(\frac{\partial \psi}{\partial \boldsymbol{F}^{e}} \boldsymbol{F}^{e T} \boldsymbol{F}^{-T}\right)=\rho_{0} \ddot{\boldsymbol{u}}
$$

Defining $\boldsymbol{T}^{f}=\boldsymbol{F}^{e T} \boldsymbol{T} \boldsymbol{F}^{-T}$ (the superscript $f$ stands for finite deformation), the rest of the derivation for the $\phi$-evolution equation stays the same as in the small deformation context. However, a new form of the layer stress, $\tau^{f}$, is required (cf Eq. (11)):

$$
\tau^{f}=\frac{1}{2 b} \int_{-b}^{b} J T_{12}^{f}(x, y, t) d y
$$


For convenience, we collect the governing equations for the finite deformation system in one place:

$$
\begin{aligned}
& \operatorname{Div} \boldsymbol{P}=\rho_{0} \ddot{\boldsymbol{u}} \\
& \phi_{t}=\frac{\left|\phi_{x}\right|^{m}}{\tilde{B}}\left(\tau+\epsilon \phi_{x x}-\tau^{b}\right) \\
& \boldsymbol{U}^{p}=\phi \boldsymbol{e}_{1} \otimes \boldsymbol{e}_{2},
\end{aligned}
$$

along with the constitutive and kinematic specifications (54 - 56).

The same simulation set-up as the small deformation case in Section 8.1.1 is used. $J$ is found to be close to 1 (maximum value over domain is 1.032) and we make the approximation of $J=1$ in our calculations. The body is supplied with a Dirichlet boundary condition that prescribes a simple shear strain of $\varepsilon_{12}$ (defined by Eq. (53)). The applied shear strain $\varepsilon_{12}$ ranges from 0.005 to 0.15 , with increments of 0.005 . The average dislocation velocity vs. applied strain is plotted, as shown in Fig. 45 . All three models $(m=0,1,2)$ give qualitatively similar profiles as the MD experiment [GG99] (Fig. 42). The curves display plateaus close to the linear elastic shear/pressure wave speeds, suggesting a definite resistance to breaking these 'sound' barriers. Increasing loads beyond these barriers causes sharp transitions in the curve. We point out that the choice of a smaller $B_{m}$ for $m=0$ is required to cluster the curves at approximately the same magnitude levels.

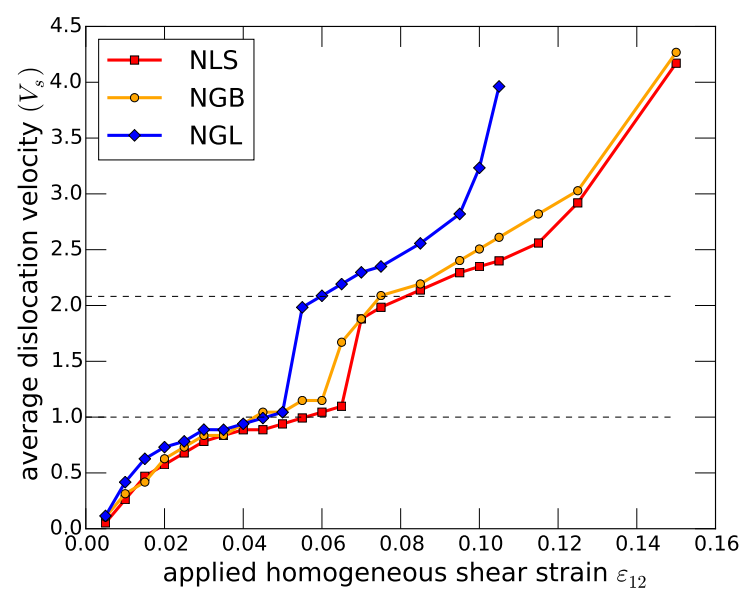

Figure 45: Dislocation velocity vs. applied strain, with inertia. Horizontal dashed lines indicate relevant acoustic wave speeds.

Figure 46 shows the magnitude of material velocity field for $\varepsilon_{12}=0.125$, bearing a qualitative similarity in the asymmetry of the pattern with the MD simulations of Gumbsch and Gao. Fig. 47 shows the shear component of Cauchy stress (post-processed from the Piola-Kirchoff stress and the deformation gradient). The dislocation accelerates from rest to supersonic speeds. The contour shows a Mach cone formed behind the dislocation tip. This suggests that the dislocation not only beats the linear elastic shear wave speed but also the wave speed of the ambient nonlinear elastic medium. But an even more interesting observation is the one demonstrated by Fig. 48 where the top panels are dislocation positions and the bottom row is the corresponding layer stress $\tau^{f}$ defined by Eq. (62): the stress-related 
part of the driving force for dislocation motion indeed keeps up with the supersonic dislocation indicating that stress wave speeds in the core region involving elastic-plastic behavior can be vastly different from the speeds of the ambient elastic medium. This emphasizes the fact that material response in the core matters even for larger scale observations and accounting only for elastic configurational forces while treating a defect core as a structureless singularity may not be adequate for many purposes, even in a partial differential equationbased theory like ours. Fig. 49 shows a plot of the hydrostatic part of the Cauchy stress (on the reference configuration). A pressure wave Mach cone forms behind the dislocation, with a Mach cone angle of about $43^{\circ}$.

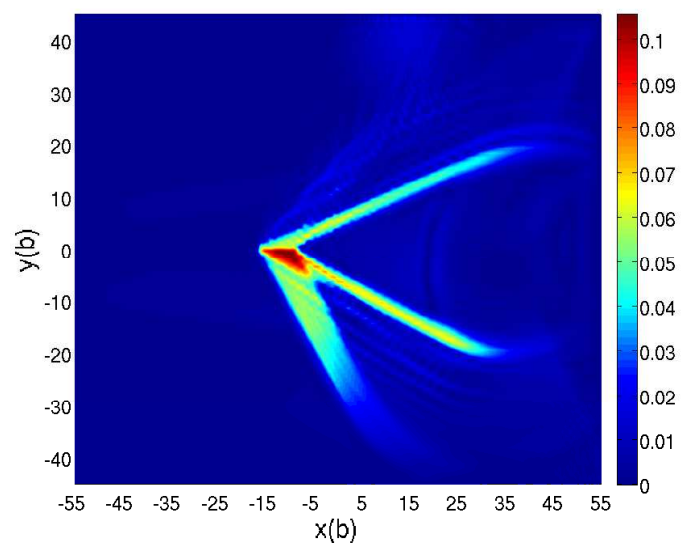

Figure 46: Material velocity field around a supersonic dislocation of speed $2.8 V_{s}$. The maximum value of material velocity is around $0.1 V_{s}$.

The dislocation velocity plotted in Fig. 45 is to be interpreted as the velocity of the image of the core under the inverse total deformation, i.e. the dislocation velocity in the reference configuration. Let us denote it as $\boldsymbol{v}^{R}$. Since the dislocation corresponds to a negative one (i.e. the 'extra half-plane of atoms' belongs to the bottom block), applied shearing in the positive $x$-direction results in dislocation motion from right to left. The dislocation velocity relative to a fixed frame, $\boldsymbol{v}_{d}$, satisfies

$$
\boldsymbol{v}^{d}=\boldsymbol{F} \boldsymbol{v}^{R}+\boldsymbol{v}^{m}
$$

where $\boldsymbol{v}^{m}$ is the material velocity. As an estimate, we interrogate the material velocity component $v_{1}^{m}$ in the case of $\varepsilon_{12}=0.125$ and find that it is relatively small compared to $v_{1}^{R}$; the maximum is approximately $0.1 V_{s}$ (as shown in Fig .46). $F_{11}$ and $F_{21}$ near the dislocation tip are approximately 1.01 and 0.01 on average. Thus, while the true dislocation velocity is expected to be lowered in magnitude somewhat from the $\boldsymbol{v}^{R}$ value, the dislocation may still be deemed as supersonic. 


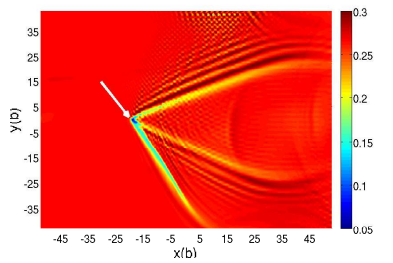

(a)

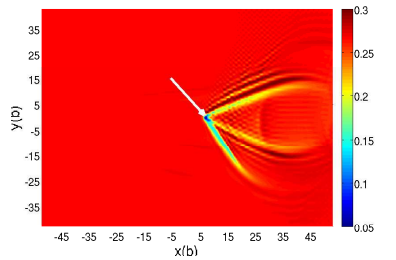

(b)

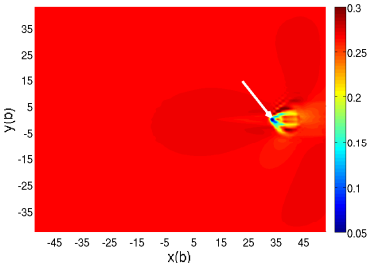

(c)

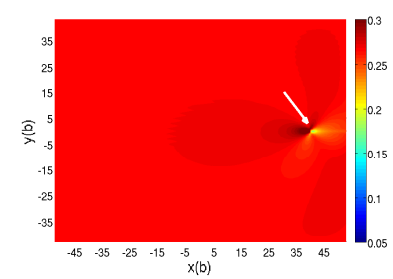

(d)

Figure 47: From right to left, Cauchy stress wave (shear component) of a dislocation moving supersonically under applied shear strain $\varepsilon_{12}=0.125$. The arrows denote the positions of the dislocation core. From (d) to (c), as the dislocation is gaining speed to supersonic the region to the left of the core is also accelerating, which generates disturbance around that area. The dislocation moving with supersonic speed ((b) and (a)) forms behind the core a shear stress mach cones.

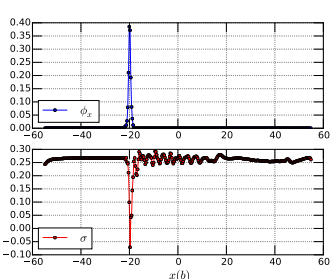

(a)

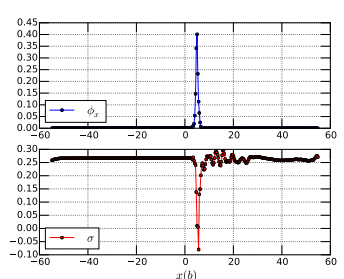

(b)

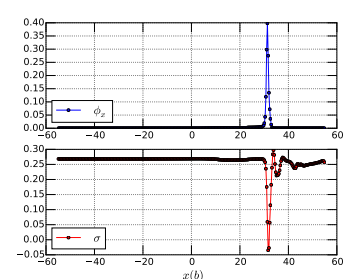

(c)

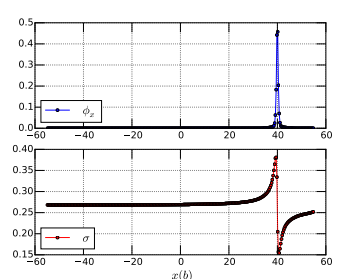

(d)

Figure 48: From right to left, the layer stress $\tau$ (bottom) moves with the dislocation(top). Waves excited by dislocation motion ripples backwards only (to the right).

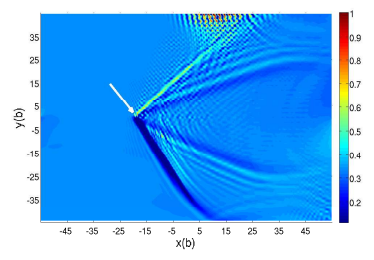

(a)

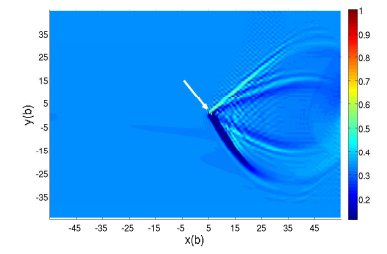

(b)

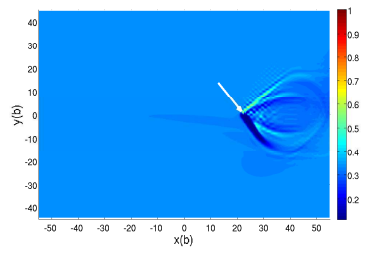

(c)

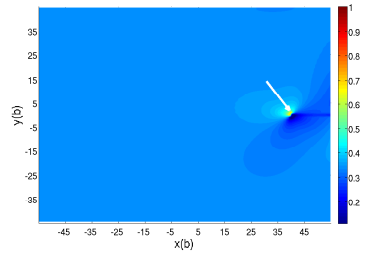

(d)

Figure 49: From right to left, hydrostatic part of Cauchy stress wave around the supersonic dislocation under applied shear strain $\varepsilon_{12}=0.125$. The stable supersonic motion of the dislocation results in a pressure wave mach cone forming behind the dislocation core.((b) and (a)).

It can be seen in the results of Fig. 47 and Fig. 49 that the Mach cone angles are obviously different between the top and the bottom blocks. In Fig. 47(b) as an example, the comparison is approximately $32.9^{\circ}$ (top) vs. $58.6^{\circ}$ (bottom). Such asymmetry is not observed in the small deformation context (Fig. 44). We want to ascribe this asymmetry to the hypothesis that the actual nonlinear wave speeds and propagation in the top and bottom blocks are 
significantly different due to the differences in the elastic strain fields there. Compared to small deformation theory where the wave speeds are simply material properties, we argue that in the calculations of Fig. 47, the stiffness largely depends on deformation states, which affects the wave speeds. The significant asymmetry between the top and bottom half body is well established, i.e., a negative edge dislocation results in a tensile stress in the horizontal direction in the vicinity of the layer in the top block and a compressive stress field in the bottom block. In Table 6 we record $\boldsymbol{E}^{e}$ of two points vertically close to the dislocation (but outside the layer) for three arbitrary time steps during the supersonic motion of the dislocation. One can see that $E_{11}^{e}$ not only has opposite signs between the top and bottom but also different absolute values; other components do not show such large asymmetry.

The separated nature of the Mach cone wings in all cases is also worthy of note; to what extent material heterogeneity (in the vertical direction) plays a role in this phenomenon remains to be explored.

\begin{tabular}{|l|c|c|c|}
\hline Time instant $\left(b / V_{s}\right)$ & $T_{1}=12.3$ & $T_{2}=8.19$ & $T_{3}=6.66$ \\
\hline strain state (top) & $\left(\begin{array}{cc}0.130 & 0.060 \\
0.060 & 0.014\end{array}\right)$ & $\left(\begin{array}{cc}0.032 & 0.121 \\
0.121 & 0.030\end{array}\right)$ & $\left(\begin{array}{cc}0.030 & 0.119 \\
0.119 & 0.030\end{array}\right)$ \\
\hline strain state (bottom) & $\left(\begin{array}{cc}-0.092 & 0.063 \\
0.063 & 0.023\end{array}\right)$ & $\left(\begin{array}{cc}-0.041 & 0.101 \\
0.101 & 0.023\end{array}\right)$ & $\left(\begin{array}{cc}-0.051 & 0.092 \\
0.092 & 0.018\end{array}\right)$ \\
\hline
\end{tabular}

Table 6: Strain states sampled at the top and bottom of a supersonic dislocation at three time instants.

\subsection{Longitudinal Shear band propagation modeled as dislocation motion}

Much like a crack-tip, a shear band is found to elongate through the motion of its tip [MD88, KW88, ZC03, GRR01]. Modeling this longitudinal extension of the band is a difficult matter since classical plasticity theory does not provide a mechanism to achieve such a dynamic extension mode, and delicate constitutive modifications have been resorted to [MLL07, ZRR96]. Here, we demonstrate this mode of shear band propagation as a direct consequence of dislocation motion, with the shear band tip interpreted as a dislocation line with a nonsingular core. In Fig. 50 we plot the current configuration of the problem presented previously in Fig. 47. The current coordinate of each node is simply a summation of the displacement and the reference coordinate. The motion of the dislocation leads to the formation of a shear band between the point $(10 b, 0)$ and $(50 b, 0)$. We use a black arrow to indicate the position of the dislocation. The corresponding (total) deformation gradient component $F_{12}$ during the extension of the shear band is plotted in Fig. 51. The highly sheared region behind the dislocation is notable.

Interestingly, for shear bands formed in this way due to the motion of a localized curl of plastic deformation (the core), the shear band width is controlled by the geometry of the core (here, its vertical extent) dragging the shear band behind it. 

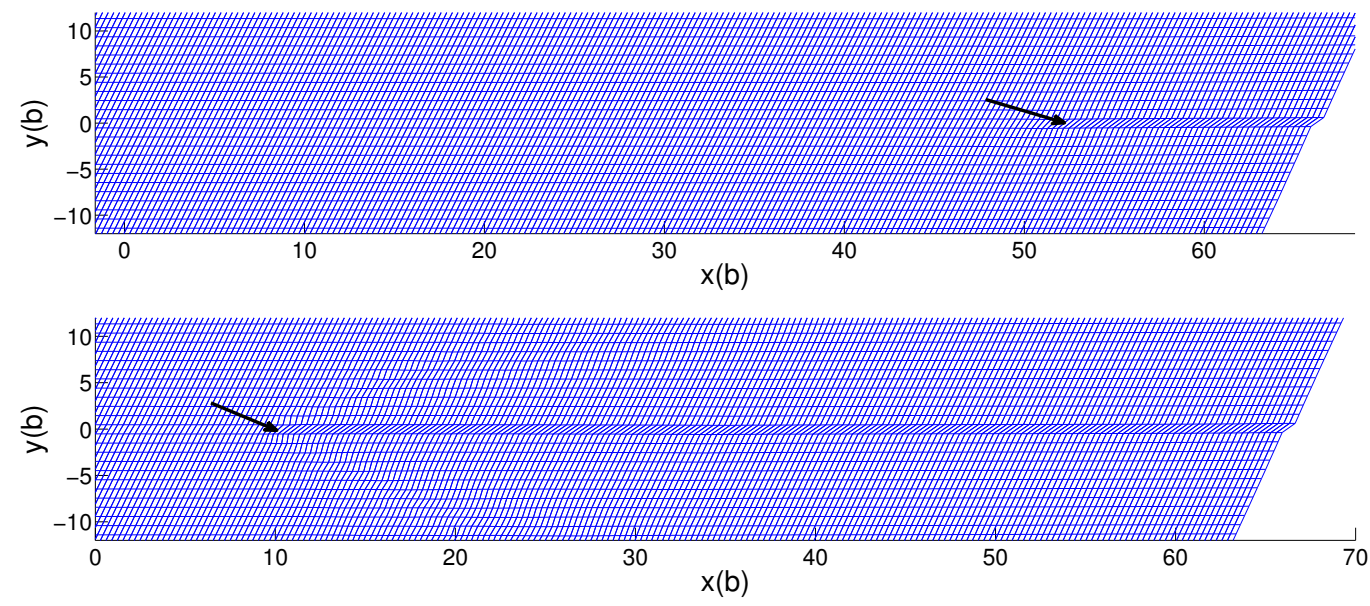

Figure 50: Shear band deformation formed behind the dislocation moving from $(50 b, 0)$ to $(10 b, 0)$.

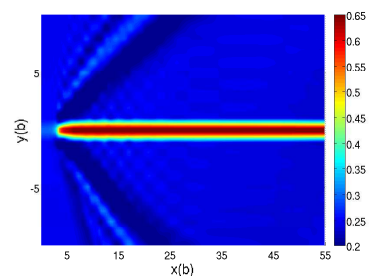

(a)

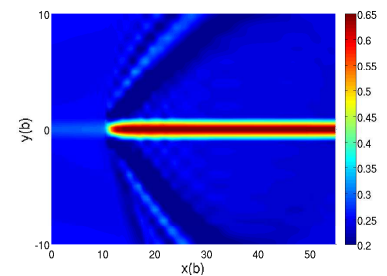

(b)

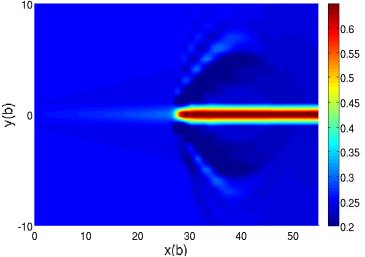

(c)

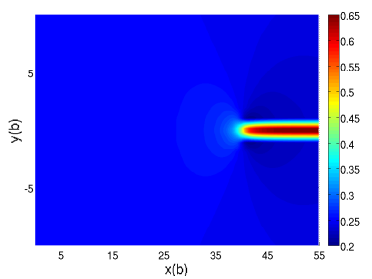

(d)

Figure 51: Deformation gradient $F_{12}$ around shear band.

\subsection{Effect of finite-speed-of-propagation of elastic waves}

The time-dependent nature of the elastic fields of dislocations cannot be ignored especially when modelling high strain rate processes. For situations involving 'shock' loadings and strain rates higher than $10^{6} \mathrm{~s}^{-1}$, utilizing quasi-static dislocation stress fields is not appropriate, even with an added mass correction to the equation of dislocation motion [HZL98]. As a consequence, a new discrete dislocation approach was developed in [GLBD $\left.{ }^{+} 13\right]$ to deal with very high strain-rate deformations. In FDM, inertia is encoded in the system naturally

through the balance law for linear momentum. The following example, directly adapted from $\left[\mathrm{GLBD}^{+} 13\right]$, demonstrates the effect of finite-speed-of-propagation of elastic waves in dislocation mechanics. The system is still governed by Eqs. (16) with $m=1$ without any further considerations of geometric or elastic nonlinearity.

A horizontal shock loading is applied on the left boundary by specifying the displacement at $x=-55 b$ as

$$
\bar{u}_{1}(\tilde{t})=\left\{\begin{array}{l}
\cos \left(\frac{\pi}{H} y\right), \tilde{t}<10 \\
0, \tilde{t}>=10
\end{array}\right.
$$


We insert a dislocation dipole in the center on the arrival of the shear stress front due to the rapid boundary loading: a crude approximation to nucleating a dislocation. Fig. 52 shows the series of shear stress contours during this process. Apart from the waves generated by the shock loading, one can clearly see that it requires a finite time for stress waves due to the dislocation dipole to propagate through the body. On the other hand, in a quasi-static setting, the elastic stress fields of the dipole would be transmitted all over the sample at the instant the dipole is nucleated even with the added mass correction (since the issue is not related to the speed of the dislocation itself, but to the propagation of elastic fields).

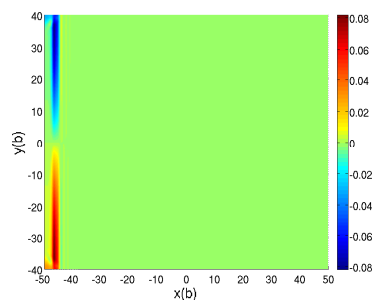

(a) $t=50 \frac{b}{V_{s}}$

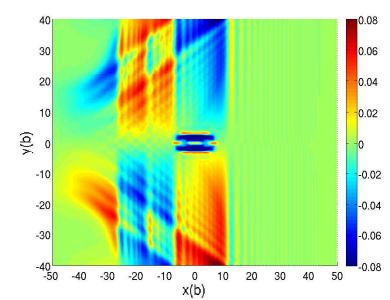

(b) $t=275 \frac{b}{V_{s}}$

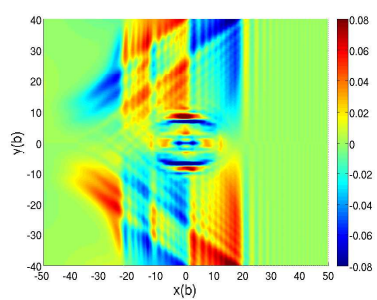

(c) $t=350 \frac{b}{V_{s}}$

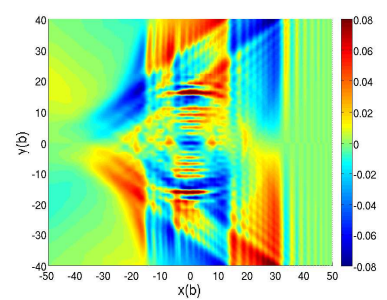

(d) $t=450 \frac{b}{V_{s}}$

Figure 52: Shear stress wave resulted by an artificially nucleated dislocation dipole.

To clearly demonstrate the finite-speed-of-propagation effect, we solve a quasi-static dipole problem as in Section 7.1.6 for the displacement field $\boldsymbol{u}_{s}$, and then superpose on $\boldsymbol{u}_{s}$ the dynamic displacement fields $\boldsymbol{u}_{d}$ taken from Fig. 52(b) and evaluate the corresponding stress field (with $\phi$ also taken from Fig. 52(b)). Compared to the 'quiet' stress field in front of the loading pulse of Fig. 52(b), an instantaneous stress field of the nucleated dipole is distributed immediately everywhere on the domain as nucleation occurs, shown in Fig. 53. The magnitude of stress at the point $(40 b, 0)$ can reach up to $0.003 \mu$ while the value is $2.8 \times 10^{-6} \mu$ at the same point in Fig. $52(\mathrm{~b})$.

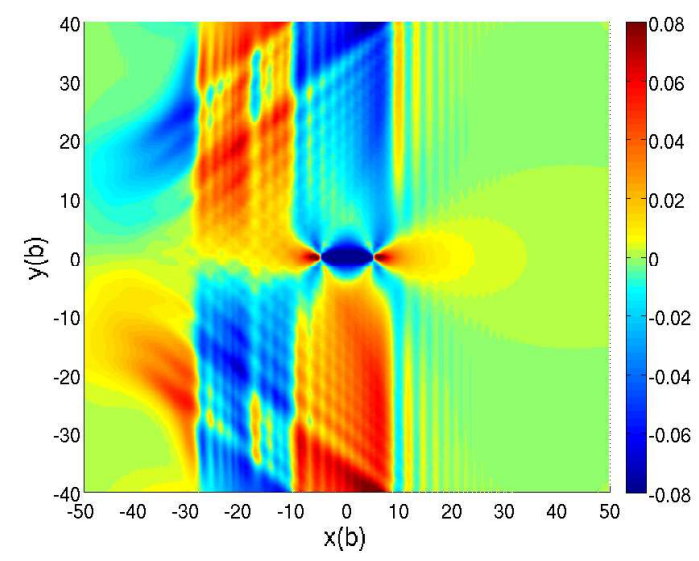

Figure 53: Quasi-static shear stress at the instant of the nucleated dislocation dipole. 


\subsection{Rupture Dynamics}

In this section we explore the possibility of utilizing FDM in modeling aspects of dynamic rupture. As early as 1970, Brune [Bru70] suggested that an earthquake might be analogous to the problem of dislocation propagation in a slip plane. Nabarro [Nab87] mentions this as well. At a very simplified level, rupture may be considered as the study of two very large blocks of material slipping with respect to each other over a thin region (relative to the size of the blocks). Based on this picture, rupture dynamics has been primarily studied as a problem of friction between two bodies. A crack is assumed to exist behind the rupture front; the crack faces are not traction free but transmit shear and normal stresses, the former limited by a friction law. Very important for this conceptual picture is the fact that the relative displacement (slip) of the crack faces is not constitutively restricted, whereas the friction law typically limits the maximum attainable shear stress that can be transmitted across the rupture layer. For a general crack, any traction profile may be imposed on the crack surfaces as Neumann boundary conditions for the equations of elasticity, this being interpreted as two conditions, one for each crack face. The friction law replaces these two conditions by demanding traction continuity and constitutively relating the magnitude of the traction to the displacement discontinuity across the layer.

On the other hand, for a classical dislocation in an elastic medium, there is again a displacement discontinuity behind the dislocation line but of fixed magnitude, and the stresses transmitted across the dislocated part of the slip plane are not limited in principle, but have to be continuous (except at the core singularity). A crack could in principle be loaded by Dirichlet boundary conditions, one for each face. The classical dislocation replaces this specified displacement boundary condition with a requirement of traction continuity and a specification of a displacement jump of fixed magnitude. An important fact about the classical dislocation picture is that the material in the wake of the moving dislocation line is indistinguishable from the intact elastic material ahead of the dislocation, thus conferring an aspect of reversibility related to material response upon dislocation motion ${ }^{7}$. Moreover, the classical notion of a dislocation and the substantial theory surrounding it related to solving for its fields allow no other possibility for material response behind the dislocation line. It is physically clear, however, that this picture cannot then be applicable to the modeling of geophysical rupture as the phenomenon definitely requires some degradation in stress response behind the rupture front.

Against this backdrop, a primary aspect of rupture dynamics is the phenomenon of self-healing. Self-healing is a conclusion deduced from observations of many earthquake records by Heaton [Hea90] which show that slip duration at any given point through which a rupture front has propagated is relatively short compared to the duration of the whole earthquake. Heaton also observed that it is a generic feature of crack models whose friction laws are of slip weakening type that short slip duration cannot be predicted. He further suggested [Hea90] slip velocity-weakening friction laws as a constitutive device that allows the accommodation of the prediction of short-slip duration. This idea has since been developed in great quantitative detail, as explained in [Ric02]. On the other hand, were a rupture front to be modeled as a dislocation line, it is a topological fact of the displacement fields of an isolated dislocation (i.e. a fixed Burgers vector) that only short slip duration can be a

\footnotetext{
${ }^{7}$ We thank Prof. J. R. Rice for emphasizing this fact.
} 
consequence behind the rupture front.

It seems to us that the dislocation picture and the crack picture are two extremes for the modeling of rupture. The assumption that there is no elastic stiffness of material behind a rupture front, even though a fault zone is of non-negligible thickness and contains, presumably, pulverized material at great depths (and therefore compressive stress), seems unrealistic to us. The shortcoming of the classical dislocation picture in dealing with the damage behind the rupture front has already been mentioned. Thus, it seems that what may be rather well-suited for the description of rupture is fundamentally a dislocation model that however allows for damage in elastic stiffness behind the rupture front. Field Dislocation Mechanics affords exactly this possibility and, as we show, interpolates between the crack and dislocation models depending on the extent of elastic damage allowed behind the rupture front.

We modify the model described in Sec. 4 leading to the system (16) as follows. We model a fault zone as a layer with a modulus $\boldsymbol{C}_{L}$ that is weaker than the outer region. We define the damaged elasticity tensor in the layer as,

$$
\boldsymbol{C}_{L}=\left(\lambda-\frac{\phi}{\bar{\phi}} \kappa\right) \boldsymbol{C}, \text { where } 0<\kappa<\lambda<1 .
$$

$\boldsymbol{C}$ is the undamaged elasticity tensor of the domain excluding the fault layer. When $\phi=0$, the non-ruptured layer has modulus $\lambda \boldsymbol{C}$, while ruptured portions of the fault have an even weaker modulus $(\lambda-\kappa) \boldsymbol{C}$. We keep all other features of the model as before. An implicit physical assumption here is that in the absence of constraints due to compatibility of total deformations, the total strains exhibited by the material in the fault zone has preferred strained states that can coexist. This is necessary. As shown before in Sec. 6.1, this is essential for a stress inducing feature as a rupture front to exist in equilibrium (as presumably it does before its motion that triggers earthquakes). For the nonconvex function $\eta$ (13), the exercise related to rupture modeling does not require a periodic potential and a triple well potential with wells at $\pm \phi, 0$ suffices.

For the purpose of illustration, we choose $\lambda=0.7$ in this section, and $\kappa$ is assumed to take the three values of $0.2,0.6,0.695$. Therefore the elastic modulus of the undamaged fault (where $\phi=0$ ) is 0.7 of the outer blocks. The fault is assumed to be completely damaged where $\phi=\bar{\phi}$ and the damaged modulus is $(0.7-\kappa) \boldsymbol{C}$, i.e., a bigger $\kappa$ represents greater damage. Within the core region the level of damage attains intermediate values as a function of $\phi$.

We start by studying the layer stress $\tau$ and $\operatorname{slip} \delta(6)$ at a fixed observation point $P$ in the path of a moving dislocation/rupture front. Specifically, the dislocation is initialized at $x=0$ and driven left by applying Dirichlet boundary condition, which prescribes a constant simple shear deformation (defined by Eq. (53)). We utilize the NLS $(m=1)$ dynamics, for no particular reason. Fig. 54(a) shows $\tau$ and $\delta$ vs. time records at $P:(-25 b, 0)$ under an applied shear strain $\varepsilon_{12}=0.03$ and $\kappa=0.2$. Starting from an NLS dislocation preequilibrium, the dislocation is driven to the left by the applied strain and passes $P$ at the time $t=490 \mathrm{~b} / V_{s}$. It is interesting to see that both quantities jump when the dislocation passes through the observation point $P$. We note that the slip stabilizes at a point once the dislocation passes through. This slip field is simply the sum of the (layer-integrated) elastic and plastic distortions at each point in the layer. The kinematics of dislocation motion 
encoded in FDM ensures that there is no evolution of plastic distortion at a point once the entire core has traversed through it, due to the absence of plastic distortion gradients behind the dislocation core.

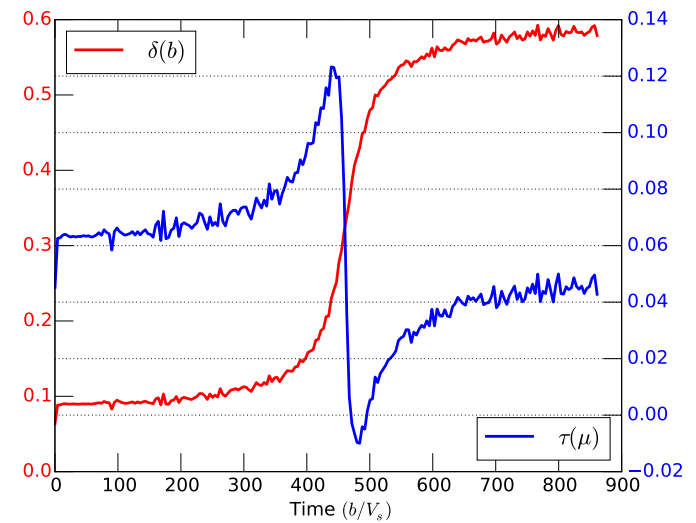

(a) $\tau$ and $\delta$
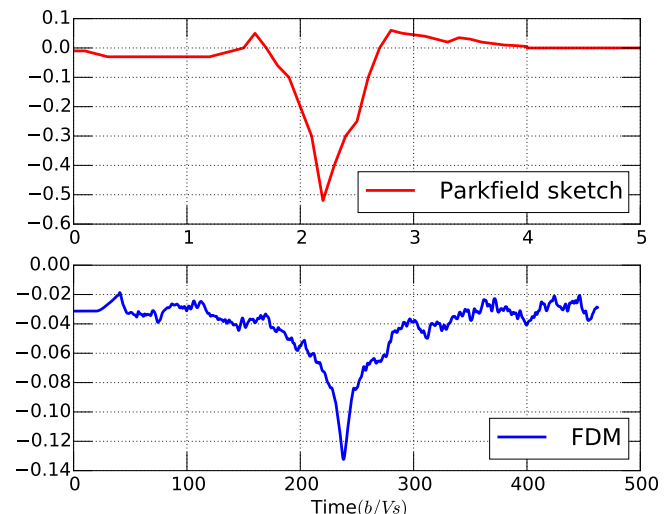

(b) $u_{2}$

Figure 54: Left: layer stress and slip calculated at a fixed point $(x=-25 b)$ in the passage of a rupture front. Right: transverse displacement at a fixed point in the rupture passage calculated by FDM model compared with sketch of the observed data recorded in the Parkfield earthquake. Sketch adapted from [AR02] (Copyright University Science Books, used with permission).

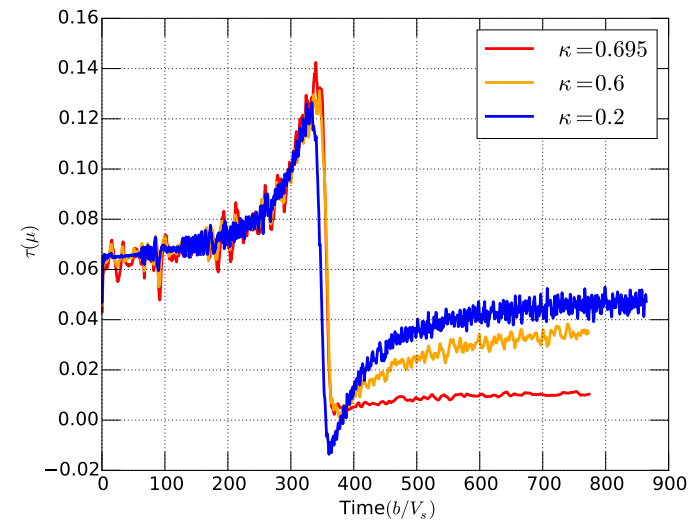

(a) $\tau$ at $x=-25 b$

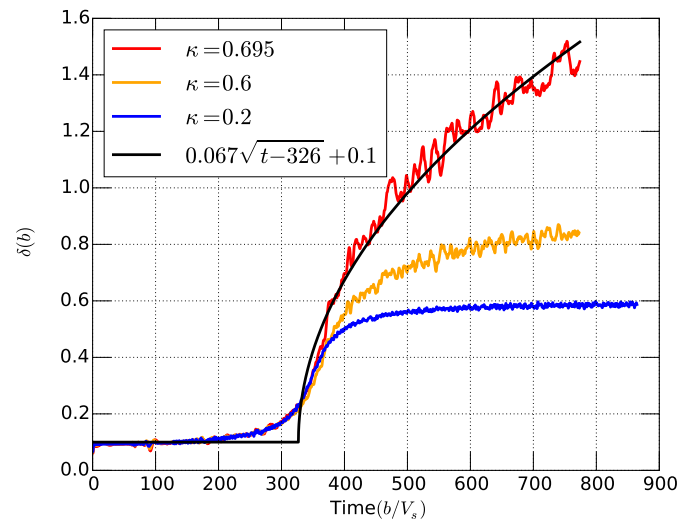

(b) $\delta$ at $x=-25 b$

Figure 55: Layer stress(left) and slip(right) plotted at a fixed point on rupture front passage with varying elasticity damage. $\kappa=0.695$ is fit by a square root function.

Fig. 54(b) shows the transverse displacement fields recorded at the same point in the case of $\kappa=0.2$. The profile qualitatively agrees with the observed form from the Parkfield earthquake sketched on the top [AR02].

Fig. 55(a) demonstrates how $\kappa$ affects the layer stress behind the rupture front. The conclusion is that the greater the 'elastic damage' behind the rupture front, the less is the 
propensity of the stress to recover after the passage of the rupture front. Fig. 55(b) shows the corresponding slip records. Note that the most damaged case $(\kappa=0.695)$ leads to a model behavior similar to a crack model, i.e., the slip at any point in the ruptured zone keeps increasing behind the rupture front as a square root function of time (unless rupture front propagation is forcibly stopped) - this is generically characteristic of all crack-like models of rupture dynamics employing slip-weakening friction laws [Hea90, DDLL05]. At the very least, then, FDM-based modeling of rupture dynamics appears to recover the response to two vastly different frictional constitutive assumptions via a simple assumption of damage of elastic modulus.

The following observations related to our preliminary foray into the modeling of rupture are in order:

- FDM naturally allows for the addition of a model of plastic deformation in the wake of the dislocation reflecting plastic straining in the absence of its spatial variation, as in classical plasticity theory. In fact, such a mode of plastic deformation is regularly used in applications of FDM at larger length scales [RA06, PDA11, FAB11]. In circumstances when the elastic modulus is substantially degraded behind the dislocation front, such an augmentation can accommodate the great variety of frictional constitutive assumptions (i.e. slip weakening, velocity weakening, rate-and-state friction) utilized in the current modeling of rupture dynamics. In ongoing work in simplified models, such a combination has been observed to give rise to stick-slip behavior.

- Due to the dependence of the elastic modulus on the plastic strain, the driving force for the dislocation (rupture front) velocity should contain a term arising from $\partial_{\phi} \boldsymbol{C}$ that we have ignored for simplicity. This term is expected to affect the observed velocity of the rupture front and not the conclusions related to the existence of short slip duration or the lack of it.

- Modeling thick faults with substantial field variations through the thickness is apparently an important issue in realistic modeling of rupture [RC07]. We note that FDM is a full 3-dimensional theory and can be invoked within the fault layer with no conceptual difficulty.

- Slow rupture fronts [RCF04, CAS99] are observed. As mentioned earlier, there is a parameter regime (i.e. large drag) for our theory where such phenomena can be modeled.

- For the sake of simplicity, we have not introduced a normal-stress dependence in rupture propagation. We note that even the simplified model of FDM utilized in this paper allows naturally for the emergence of a normal-stress dependence in the rupture velocity, as shown in the development of Section 4.

\section{Concluding Remarks}

It is perhaps fair to say that at the current time the classical theory of dislocations [HL82] and the theory of elastoplasticity [Lub01] appear to be disparate subjects. We hope to have 
demonstrated in this paper that FDM, which is nothing but a theory of elastoplasticity with a fancy, but physically rigorous, evolution equation for the plastic distortion, encompasses a large class of key features of classical dislocation theory in both its extensively developed static and its barely developed time-dependent aspects. This observation is particularly important in situations involving dynamics with inertia where the classical theory has severe conceptual limitations.

The computational methods of approximation that we use to study FDM are versatile and capable of natural extension to 3 space dimensions, representation of multiple slip, finite deformations (in full generality), elastic anisotropy, arbitrary loadings, and complex domains. This is desirable, both from the scientific and engineering points of view. In a sense, the theory and methods we propose for the study of dislocation mechanics have the potential of bringing the same type of efficiency and generality to the study of the physical subject as the finite element method did to the study of elasticity theory. Indeed, a reading of papers like that of Eshelby, Frank, and Nabarro [EFN51] leaves one marveling at the creativity of the authors; however, the techniques utilized are very special. Our approximation methods are accessible to anyone with training in the standard repertoire of computational mechanics with a desire to learn about dislocation mechanics, and this is as much a result of the theoretical framework that is employed.

Starting from the pioneering work of Aifantis [Aif84] there has been a great emphasis in the solid mechanics community in developing models of strain gradient plasticity in the last 30 years or so, often to make connection with dislocation mechanics. It is again perhaps fair to say that while many models have emerged, none can lay claim to being a theory for the mechanics of dislocations in any distinguished limit, whether for individual or collective behavior. In contrast, we have shown that the equations of FDM, which may also be considered as a gradient-plasticity model in the form (5), but of very different structure compared to bona-fide standard strain gradient plasticity theories, can make the claim at the level of representing a great variety of individual dislocation behavior. There are important questions of time-dependent homogenization related to the modeling of mesoscopic and macroscopic plasticity that remain to be addressed, but we take satisfaction in the fact that a correct pde-based microscopic standpoint has been established. Moreover, work based on heuristic models of collective behavior developed on FDM as the underlying microscopic theory has shown promise [AR06, RA06, PDA11, ABM08, FAB11, $\left.\mathrm{CCP}^{+} 13\right]$, including the demonstration of how standard elastoplasticity theory can be incorporated within the theoretical structure if so desired.

The most important future work related to FDM that remains for us to execute is a robust computational implementation of the 3-d theory without restriction to special 'slip layers,' with slip-system like behavior being an outcome. This will require careful design of the energetics related to slip (i.e. the $\eta$ function) and, very importantly, an accurate and stable numerical scheme for the $\boldsymbol{U}^{p}$ evolution equation which will need to be capable of representing dynamic, string-like, nonsingular, stress-inducing localizations in the field. Despite appearances arising from the simplified ansatz we have utilized in this paper, the fundamental 3-d equations of FDM are not a variation on reaction diffusion systems or scalar, hyperbolic, conservation laws; see [AT11] for illustration of this issue. These are interesting, but tractable, challenges. The question related to energetics also has an important philosophical angle in that it should be defined purely in terms of quantities identifiable from the 
current state without reference to any reference configuration in the past, and the plastic distortion does not readily lend itself to such definition. It is very likely that resolution will lie in a careful adaptation of ideas from [RBS92, Vit68, SW03, Zim04, Par10, NP14].

\section{Acknowledgment}

This research was supported in part by the National Science Foundation through grant CMMI 1435624. Generous grants of computer time and help from Pittsburgh Supercomputing Institute are gratefully acknowledged.

\section{References}

[ABM08] Amit Acharya, Armand Beaudoin, and Ron Miller. New perspectives in plasticity theory: dislocation nucleation, waves, and partial continuity of plastic strain rate. Mathematics and Mechanics of Solids, 13(3-4):292-315, 2008.

[Ach01] Amit Acharya. A model of crystal plasticity based on the theory of continuously distributed dislocations. Journal of the Mechanics and Physics of Solids, 49(4):761-784, 2001.

[Ach03] Amit Acharya. Driving forces and boundary conditions in continuum dislocation mechanics. Proceedings of the Royal Society of London. Series A: Mathematical, Physical and Engineering Sciences, 459(2034):1343-1363, 2003.

[Ach04] Amit Acharya. Constitutive analysis of finite deformation field dislocation mechanics. Journal of the Mechanics and Physics of Solids, 52(2):301-316, 2004.

[Ach07] Amit Acharya. Jump condition for GND evolution as a constraint on slip transmission at grain boundaries. Philosophical magazine, 87(8-9):1349-1359, 2007.

[Ach10] Amit Acharya. New inroads in an old subject: plasticity, from around the atomic to the macroscopic scale. Journal of the Mechanics and Physics of Solids, 58(5):766-778, 2010.

[Ach11] Amit Acharya. Microcanonical entropy and mesoscale dislocation mechanics and plasticity. Journal of Elasticity, 104(1-2):23-44, 2011.

[AHLBM06] Olivier Alvarez, Philippe Hoch, Yann Le Bouar, and Régis Monneau. Dislocation dynamics: short-time existence and uniqueness of the solution. Archive for Rational Mechanics and Analysis, 181(3):449-504, 2006.

[Aif84] E. C. Aifantis. On the microstructural origin of certain inelastic models. Journal of Engineering Materials and Technology, 106(4):326-330, 1984. 
[AMZ10] Amit Acharya, Karsten Matthies, and Johannes Zimmer. Travelling wave solutions for a quasilinear model of field dislocation mechanics. Journal of the Mechanics and Physics of Solids, 58(12):2043-2053, 2010.

[AR02] Keiiti Aki and Paul G. Richards. Quantitative Seismology. University Science Books, 2002.

[AR06] Amit Acharya and Anish Roy. Size effects and idealized dislocation microstructure at small scales: predictions of a phenomenological model of mesoscopic field dislocation mechanics: Part I. Journal of the Mechanics and Physics of Solids, 54(8):1687-1710, 2006.

[AT11] Amit Acharya and Luc Tartar. On an equation from the theory of field dislocation mechanics. Bollettino dell' Unione Matematica Italiana, 9(IV):409-444, 2011.

[AZ14] Amit Acharya and Xiaohan Zhang. From dislocation motion to an additive velocity gradient decomposition, and some simple models of dislocation dynamics. In Ta-Tsien Li and Ph. G. Ciarlet, editors, Proceedings of the International Conference in honor of Luc Tartar on Nonlinear and Multiscale Partial Differential Equations: Theory, Numerics and Applications; Series in Contemporary Applied Mathematics. Higher Education Press (Beijing) and World Scientific, Singapore, 2014.

[BCBK06] V. V. Bulatov, W. Cai, R. Baran, and K. Kang. Geometric aspects of the ideal shear resistance in simple crystal lattices. Philosophical Magazine, 86(2526):3847-3859, 2006.

$\left[\mathrm{BJC}^{+} 01\right] \quad$ V.V. Bulatov, J.F. Justo, Wei Cai, Sidney Yip, A.S. Argon, T. Lenosky, M. de Koning, and T. Diaz de la Rubia. Parameter-free modelling of dislocation motion: the case of silicon. Philosophical Magazine A, 81(5):1257-1281, 2001.

[Bru70] James N. Brune. Tectonic stress and the spectra of seismic shear waves from earthquakes. Journal of Geophysical Research, 75(26):4997-5009, 1970.

[CAS99] Luca Crescentini, Antonella Amoruso, and Roberto Scarpa. Constraints on slow earthquake dynamics from a swarm in central Italy. Science, 286(5447):21322134, 1999.

[CAWB06] Wei Cai, Athanasios Arsenlis, Christopher R. Weinberger, and Vasily V. Bulatov. A non-singular continuum theory of dislocations. Journal of the Mechanics and Physics of Solids, 54(3):561-587, 2006.

[CCHO97] A. Carpio, S. J. Chapman, S. D. Howison, and J. R. Ockendon. Dynamics of line singularities. Philosophical Transactions of the Royal Society of London. Series A: Mathematical, Physical and Engineering Sciences, 355(1731):20132024, 1997. 
$\left[\mathrm{CCP}^{+} 13\right] \quad$ Yong S. Chen, Woosong Choi, Stefanos Papanikolaou, Matthew Bierbaum, and James P. Sethna. Scaling theory of continuum dislocation dynamics in three dimensions: Self-organized fractal pattern formation. International Journal of Plasticity, 46:94-129, 2013.

[CCV01] Ana Carpio, S. Jonathan Chapman, and J. J. L. Velázquez. Pile-up solutions for some systems of conservation laws modelling dislocation interaction in crystals. SIAM Journal on Applied Mathematics, 61(6):2168-2199, 2001.

[CP89] Jack Carr and Robert L. Pego. Metastable patterns in solutions of $u_{t}=\epsilon^{2} u_{x x}-$ $f(u)$. Communications on Pure and Applied Mathematics, 42(5):523-576, 1989.

[DAZM13] Amit Das, Amit Acharya, Johannes Zimmer, and Karsten Matthies. Can equations of equilibrium predict all physical equilibria? A case study from Field Dislocation Mechanics. Mathematics and Mechanics of Solids, 18:801-820, 2013.

[DDLL05] Steven M. Day, Luis A. Dalguer, Nadia Lapusta, and Yi Liu. Comparison of finite difference and boundary integral solutions to three-dimensional spontaneous rupture. Journal of Geophysical Research: Solid Earth (1978-2012), 110(B12), 2005.

[Den04] Christophe Denoual. Dynamic dislocation modeling by combining Peierls Nabarro and Galerkin methods. Physical Review B, 70(2):024106, 2004.

[EFN51] J.D. Eshelby, F.C. Frank, and F.R.N. Nabarro. The equilibrium of linear arrays of dislocations. Philosophical Magazine, 42(327):351-364, 1951.

[FAB11] C. Fressengeas, A. Acharya, and A. J. Beaudoin. Dislocation mediated continuum plasticity: Case studies on modeling scale dependence, scale-invariance, and directionality of sharp yield-point. In S. Ghosh and D. M. Dimiduk, editors, Computational Methods for Microstructure-Property Relationships. Springer, 2011.

[Fox66] N. Fox. A continuum theory of dislocations for single crystals. IMA Journal of Applied Mathematics, 2(4):285-298, 1966.

[Fre98] L. B. Freund. Dynamic fracture mechanics. Cambridge University Press, 1998.

[GAM15] Akanksha Garg, Amit Acharya, and Craig E. Maloney. A study of conditions for dislocation nucleation in coarser-than-atomistic scale models. Journal of the Mechanics and Physics of Solids, 75:76-92, 2015.

[GG99] Peter Gumbsch and Huajian Gao. Dislocations faster than the speed of sound. Science, 283(5404):965-968, 1999.

$\left[\mathrm{GLBD}^{+} 13\right]$ Beñat Gurrutxaga-Lerma, Daniel S. Balint, Daniele Dini, Daniel E. Eakins, and Adrian P. Sutton. A dynamic discrete dislocation plasticity method for the simulation of plastic relaxation under shock loading. Proceedings of the Royal Society A: Mathematical, Physical and Engineering Science, 469(2156), 2013. 
[GRR01] P. R. Guduru, A. J. Rosakis, and G. Ravichandran. Dynamic shear bands: an investigation using high speed optical and infrared diagnostics. Mechanics of Materials, 33(7):371-402, 2001.

[Hea90] Thomas H. Heaton. Evidence for and implications of self-healing pulses of slip in earthquake rupture. Physics of the Earth and Planetary Interiors, 64(1):120, 1990.

[HL82] John P. Hirth and Jens Lothe. Theory of dislocations. John Wiley \& Sons, 1982.

[HZL98] J.P. Hirth, H.M. Zbib, and J. Lothe. Forces on high velocity dislocations. Modelling and Simulation in Materials Science and Engineering, 6(2):165, 1998.

[IRG15] Mrinal Iyer, Balachandran Radhakrishnan, and Vikram Gavini. Electronicstructure study of an edge dislocation in aluminum and the role of macroscopic deformations on its energetics. Journal of the Mechanics and Physics of Solids, 76:260-275, 2015.

[KBC12] Keonwook Kang, Vasily V. Bulatov, and Wei Cai. Singular orientations and faceted motion of dislocations in body-centered cubic crystals. Proceedings of the National Academy of Sciences, 109(38):15174-15178, 2012.

$\left[\mathrm{KCC}^{+} 92\right]$ Ladislas P. Kubin, G. Canova, M. Condat, Benoit Devincre, V. Pontikis, and Yves Bréchet. Dislocation microstructures and plastic flow: a 3d simulation. Solid State Phenomena, 23:455-472, 1992.

[KCO02] Marisol Koslowski, Alberto M. Cuitino, and Michael Ortiz. A phase-field theory of dislocation dynamics, strain hardening and hysteresis in ductile single crystals. Journal of the Mechanics and Physics of Solids, 50(12):2597-2635, 2002.

[Krö81] Ekkehart Kröner. Continuum theory of defects. In R. Balian and M. Kleman et al., editors, Physics of Defects, Les-Houches Summer School, pages 217-315. North-Holland, Amsterdam, 1981.

[KW88] J. F. Kalthoff and S. Winkler. Failure mode transitions at high rates of loading. In L. W. Meyer C. Y. Chiem, H. D. Kunze, editor, Proceedings of the International Conference on Impact Loading and Dynamic Behavior of Materials, pages 43-56. DeutscheGesselschaft fur Metallkunde, 1988.

[LH59] J. Lothe and J.P. Hirth. Dislocation dynamics at low temperatures. Physical Review, 115(3):543, 1959.

[LKBK00] Gang Lu, Nicholas Kioussis, Vasily V. Bulatov, and Efthimios Kaxiras. The Peierls-Nabarro model revisited. Philosophical Magazine Letters, 80(10):675682,2000 . 
[LS08] Surachate Limkumnerd and James P. Sethna. Shocks and slip systems: Predictions from a mesoscale theory of continuum dislocation dynamics. Journal of the Mechanics and Physics of Solids, 56(4):1450-1459, 2008.

[Lub01] Vlado A. Lubarda. Elastoplasticity theory. CRC press, 2001.

[Mar11] Xanthippi Markenscoff. Comment on dynamic Peierls-Nabarro equations for elastically isotropic crystals. Physical Review B, 83(5):056101, 2011.

[MBW98] A. B. Movchan, R. Bullough, and J. R. Willis. Stability of a dislocation: discrete model. European Journal of Applied Mathematics, 9(04):373-396, 1998.

[MD88] A. Marchand and J. Duffy. An experimental study of the formation process of adiabatic shear bands in a structural steel. Journal of the Mechanics and Physics of Solids, 36(3):251-283, 1988.

[MLL07] Sergey N. Medyanik, Wing Kam Liu, and Shaofan Li. On criteria for dynamic adiabatic shear band propagation. Journal of the Mechanics and Physics of Solids, 55(7):1439-1461, 2007.

[MPBO98] Ron Miller, Rob Phillips, Glen Beltz, and Michael Ortiz. A non-local formulation of the Peierls dislocation model. Journal of the Mechanics and Physics of Solids, 46(10):1845-1867, 1998.

[Mur63] T. Mura. Continuous distribution of moving dislocations. Philosophical Magazine, 8(89):843-857, 1963.

[Nab47] F.R.N. Nabarro. Dislocations in a simple cubic lattice. Proceedings of the Physical Society, 59(2):256, 1947.

[Nab87] F. R. N. Nabarro. Theory of crystal dislocations. Dover, 1987.

[NP14] Rachel Nicks and Gareth Parry. Group elastic symmetries common to continuum and discrete defective crystals. Journal of Elasticity, 115(2):131-156, 2014.

[Par10] Gareth P. Parry. Elastic symmetries of defective crystals. Journal of Elasticity, 101(1):101-120, 2010.

[PDA11] Saurabh Puri, Amit Das, and Amit Acharya. Mechanical response of multicrystalline thin films in mesoscale field dislocation mechanics. Journal of the Mechanics and Physics of Solids, 59(11):2400-2417, 2011.

[PDT10] Y-P Pellegrini, Christophe Denoual, and Lev Truskinovsky. Phase-field modeling of nonlinear material behavior. In IUTAM symposium on variational concepts with applications to the mechanics of materials, pages 209-220. Springer, 2010.

[Pei40] R. Peierls. The size of a dislocation. Proceedings of the Physical Society, 52(1):34-37, 1940. 
[Pel10] Yves-Patrick Pellegrini. Dynamic Peierls-Nabarro equations for elastically isotropic crystals. Physical Review B, 81(2):024101, 2010.

[Pel11] Yves-Patrick Pellegrini. Reply to comment on dynamic Peierls-Nabarro equations for elastically isotropic crystals. Physical Review B, 83(5):056102, 2011.

[Pic02] R.C. Picu. The Peierls stress in non-local elasticity. Journal of the Mechanics and Physics of Solids, 50(4):717-735, 2002.

[PLSG14] Giacomo Po, Markus Lazar, Dariush Seif, and Nasr Ghoniem. Singularity-free dislocation dynamics with strain gradient elasticity. Journal of the Mechanics and Physics of Solids, 68:161-178, 2014.

[RA06] Anish Roy and Amit Acharya. Size effects and idealized dislocation microstructure at small scales: predictions of a phenomenological model of mesoscopic field dislocation mechanics: Part II. Journal of the Mechanics and Physics of Solids, 54(8):1711-1743, 2006.

[RBS92] J. R. Rice, G. E. Beltz, and Y. Sun. Peierls framework for dislocation nucleation from a crack tip. In Topics in fracture and fatigue, pages 1-58. Springer, 1992.

[RC07] James R. Rice and Massimo Cocco. Seismic fault rheology and earthquake dynamics. Tectonic Faults: Agents of Change on a Dynamic Earth, pages 99137, 2007.

[RCF04] Shmuel M. Rubinstein, Gil Cohen, and Jay Fineberg. Detachment fronts and the onset of dynamic friction. Nature, 430(7003):1005-1009, 2004.

[Ric71] James R. Rice. Inelastic constitutive relations for solids: an internal-variable theory and its application to metal plasticity. Journal of the Mechanics and Physics of Solids, 19(6):433-455, 1971.

[Ric02] James R. Rice. New perspectives on crack and fault dynamics. In Mechanics for a New Mellennium, pages 1-24. Springer, 2002.

[RLBF03] D. Rodney, Y. Le Bouar, and A. Finel. Phase field methods and dislocations. Acta Materialia, 51(1):17-30, 2003.

[RSC99] A.J. Rosakis, O. Samudrala, and D. Coker. Cracks faster than the shear wave speed. Science, 284(5418):1337-1340, 1999.

[Sch99] G. Schoeck. Peierls energy of dislocations: a critical assessment. Physical Review Letters, 82(11):2310, 1999.

[SHZG11] Stefan Sandfeld, Thomas Hochrainer, Michael Zaiser, and Peter Gumbsch. Continuum modeling of dislocation plasticity: Theory, numerical implementation, and validation by discrete dislocation simulations. J. Mater. Res, 26(5):624, 2011. 
[SW03] C. Shen and Y. Wang. Phase field model of dislocation networks. Acta materialia, 51(9):2595-2610, 2003.

[VBAF06] S. N. Varadhan, A. J. Beaudoin, A. Acharya, and C. Fressengeas. Dislocation transport using an explicit Galerkin/Least-squares formulation. Modelling and Simulation in Materials Science and Engineering, 14(7):1245, 2006.

[Vit68] V. Vitek. Intrinsic stacking faults in body-centred cubic crystals. Philosophical Magazine, 18(154):773-786, 1968.

[WH74] W. W. Wood and A. K. Head. The motion of dislocations. Proceedings of the Royal Society of London. A. Mathematical and Physical Sciences, 336(1605):191-209, 1974.

[Wil65] J. R. Willis. Dislocations and inclusions. Journal of the Mechanics and Physics of Solids, 13(6):377-395, 1965.

[Wil67] John R. Willis. Second-order effects of dislocations in anisotropic crystals. International Journal of Engineering Science, 5(2):171-190, 1967.

[WJCK01] Yu U. Wang, Y. M. Jin, A. M. Cuitino, and A. G. Khachaturyan. Nanoscale phase field microelasticity theory of dislocations: model and 3d simulations. Acta Materialia, 49(10):1847-1857, 2001.

[WL10] Yunzhi Wang and Ju Li. Phase field modeling of defects and deformation. Acta Materialia, 58(4):1212-1235, 2010.

[XCSE03] Yang Xiang, Li-Tien Cheng, David J. Srolovitz, and Weinan E. A level set method for dislocation dynamics. Acta Materialia, 51(18):5499-5518, 2003.

[YGVdG04] S. Yefimov, I. Groma, and E. Van der Giessen. A comparison of a statisticalmechanics based plasticity model with discrete dislocation plasticity calculations. Journal of the Mechanics and Physics of Solids, 52(2):279-300, 2004.

[ZC03] Z. Zhang and R. J. Clifton. Shear band propagation from a crack tip. Journal of the Mechanics and Physics of Solids, 51(11):1903-1922, 2003.

[Zim04] Johannes Zimmer. Stored energy functions for phase transitions in crystals. Archive for Rational Mechanics and Analysis, 172(2):191-212, 2004.

[ZRR96] M. Zhou, G. Ravichandran, and A.J. Rosakis. Dynamically propagating shear bands in impact-loaded prenotched plates - II. numerical simulations. Journal of the Mechanics and Physics of Solids, 44(6):1007-1032, 1996. 